BNL-114540-2017-IR

CBETA/008

January 2017

\title{
Halbach Magnets for CBETA
}

\author{
D. Trbojevic et al.
}

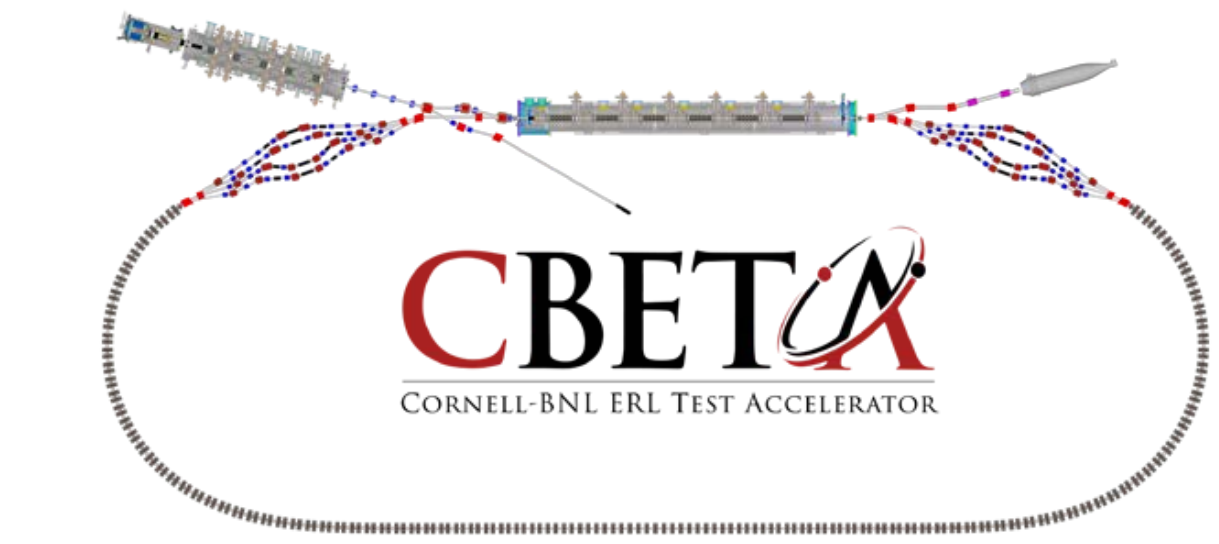

Collider-Accelerator Department, Brookhaven National Laboratory, Upton NY 11973

U.S. Department of Energy

Office of Science, Office of Nuclear Physics

Cornell Laboratory for Accelerator-Based Sciences and Education, Ithaca, NY 14850

Funded by NYSERDA contract 102192

Notice: This document has been authorized by employees of Brookhaven Science Associates, LLC under Contract No. DE-SC0012704 with the U.S. Department of Energy. The United States Government retains a non-exclusive, paid-up, irrevocable, world-wide license to publishor reproduce the published form of this document, or allow others to do so, for United States Government purposes. 


\section{DISCLAIMER}

This report was prepared as an account of work sponsored by an agency of the United States Government. Neither the United States Government nor any agency thereof, nor any of their employees, nor any of their contractors, subcontractors, or their employees, makes any warranty, express or implied, or assumes any legal liability or responsibility for the accuracy, completeness, or any third party's use or the results of such use of any information, apparatus, product, or process disclosed, or represents that its use would not infringe privately owned rights. Reference herein to any specific commercial product, process, or service by trade name, trademark, manufacturer, or otherwise, does not necessarily constitute or imply its endorsement, recommendation, or favoring by the United States Government or any agency thereof or its contractors or subcontractors. The views and opinions of authors expressed herein do not necessarily state or reflect those of the United States Government or any agency thereof. 


\section{Halbach Magnets for CBETA}

\section{TECHNICAL REVIEW}

1.1 Advantages of the Halbach magnets for CBETA

1.2 Required Goals of the New Halbach Magnet Design

1.3 Lattice design

1.3.1 Orbit offsets in the $150 \mathrm{MeV}$ design for Hybrid and Halbach magnet

1.3.2 Halbach Magnet Design with “OPERA 3D” and Tests from Tracking

\subsection{Overall Assemblies and Quantity}

1.5 Assembly procedure

1.6 Cross-Sections with Dimensions

1.7 Shimming Method

1.8 Vacuum Round Pipe Design (Four Button BPM's)

1.9 Results from Prototype Halbach Magnets (250 MeV)

1.9.1 Comparison of Features vs. Iron Poled Magnets

1.9.2 Halbach Magnet Design

1.9.3 Magnet Simulation and Codes

1.9.4 Tracking and Compatibility with FFAG Lattice

1.9.5 Window-Frame Correctors

1.9.6 The B field of a Window-Frame Electromagnet

1.9.7 Halbach Magnet R\&D and Shimming Results

1.9.8 Repeatability of Un-shimmed Halbach Magnets

1.9.9 Field Quality Improvement after Iron Wire Shimming

1.9.10 Measurements of the Neighboring Magnet's Effect

1.9.11. Plans for "future" Halbach Magnet

1.10 Response to the March 2016 Magnet Reviewers

\section{COST}

2.1 Cost Comparison

2.2 The Lowest Estimated

2.3 Sales Quotes

\section{RISKS and MITIGATIONS}

\section{SCHEDULE}

\subsection{FIRST GIRDER PLAN}

4.1.1 First Halbach Magnet Production

4.1.2 Measurements and Corrections

4.1.3 First Girder Test

4.2 FULL MAGNET PRODUCTION AND INTEGRATION

\section{SUMMARY}




\section{Halbach Magnets for CBETA (150MeV)}

A principle of the non-scaling Fixed Field Alternating Gradient (NS-FFAG) is that different energy beam has orbit oscillations $\Delta x$ around the central circular orbit in both positive and negative direction within a small radial aperture as: $\Delta x=D_{x}{ }^{*} \delta p / p$. For the central circular orbit $\Delta x=0$, or for the combined function magnets the field is equal to $B_{o}\left(B(x)=B_{o}+G * x\right)$. The smallest orbit offsets $\Delta x$ are obtained when the defocusing magnet provides most of the bending for the central energy, while the focusing magnet could be even the regular quadrupole with the central orbit in the middle. Stable orbits for a very large energy range [in the case of CBETA this is 4 times in energy], is obtained using opposite polarity magnets producing linear magnetic fields, small dispersion, and very strong focusing.

The initial CBETA lattice design together with the Halbach magnets was made with the maximum energy of $E_{\max }=286 \mathrm{MeV}$, assuming the superconducting linac energy of $70 \mathrm{MeV}$, with the injection energy of 6 $\mathrm{MeV}$. Due to uncertainties the linac operating energy was reduced from $70 \rightarrow 61 \mathrm{MeV}$ and the maximum achievable energy was defined as $E_{\max }=250 \mathrm{MeV}$, with energies after each pass: 67, 128, 189, and $250 \mathrm{MeV}$. Twelve prototype Halbach magnets were built, assembled and measured with and without compensation iron wires. As the Hybrid magnets - radially misplaced quadrupoles - could not provide strong enough magnetic field for $250 \mathrm{MeV}$, an attempt was made with the NS-FFAG lattice reducing the maximum energy to $E_{\max }=200 \mathrm{MeV}$. Again, due to a problem of not having enough safety margins by the hybrid magnets the CBETA maximum energy was reduced to $E_{\max }=150 \mathrm{MeV}$ and the base line lattice is designed, with larger drifts: one equal to $7 \mathrm{~cm}$ and $12 \mathrm{~cm}$. We have presently designed new Halbach focusing and defocusing combined function magnets of the same size as the hybrid iron magnets to replace them without affecting the base line design at all. There were two kind of permanent magnets proposed for the CBETA project: Hybrid-iron dominated quadrupole, and the Halbach type of magnets.

\subsection{Advantages of the Halbach magnets for CBETA}

1.1.1 The Halbach magnets (focusing quadrupole and defocusing combined function magnet) make the most efficient way for using the permanent magnet material or it is the smallest possible permanent magnet material volume used to create the combined function magnets. This is especially true for the NS-FFAG, as the different energy orbits are contained in the center of both kinds of magnets.

1.1.2 Quality of the magnetic field (shown by measurements is excellent). The largest values of all the magnetic multipoles, up to the $20^{\text {th }}$ pole, are less than a unit $\left(B_{N} / B_{0}=10^{-4} @ 1 \mathrm{~cm}\right)$ with a maximum variation of the gradient less than $\Delta G_{\max }<0.2 \%$. There is no need for magnets to be radially shifted as the requested combined function field of the magnet is produced by the magnet design. The magnet is used the most effective way as the required combined function magnetic field is provided in the center of the beam orbit range. The combined function magnetic field in the hybrid magnet case is obtained by radially shifting the quadrupoles.

1.1.3 No cross talk between the magnets as the field is contained within the magnet with the fringe fields from the neighboring magnet not affecting at all the field within the magnet. The superposition of 
the magnetic fields shown in the 3D OPERA calculations are also confirmed by the magnetic measurements with two Halbach magnets separated $6 \mathrm{~cm}$ apart from each other.

1.1.4 The iron corrector frame is a very simple correction system. It is place around the magnet with very small effect on the Halbach magnetic field as the relative permeability is $\mu_{R E L}=1.1$. The dipole corrector's effect on the quadrupole field is $0.2 \%$ confirmed by the measurements with and without the corrector frame around the magnet.

1.1.5 Bent round pipe fits through both Halbach magnets with enough clearance, simplifying the whole CBETA design and installation and allowing 4 button BPM's as well. This can bring savings in the BPM electronics from $\$ 660,000.00$ to $\$ 330,000.00$ as the number of boards is reduced.

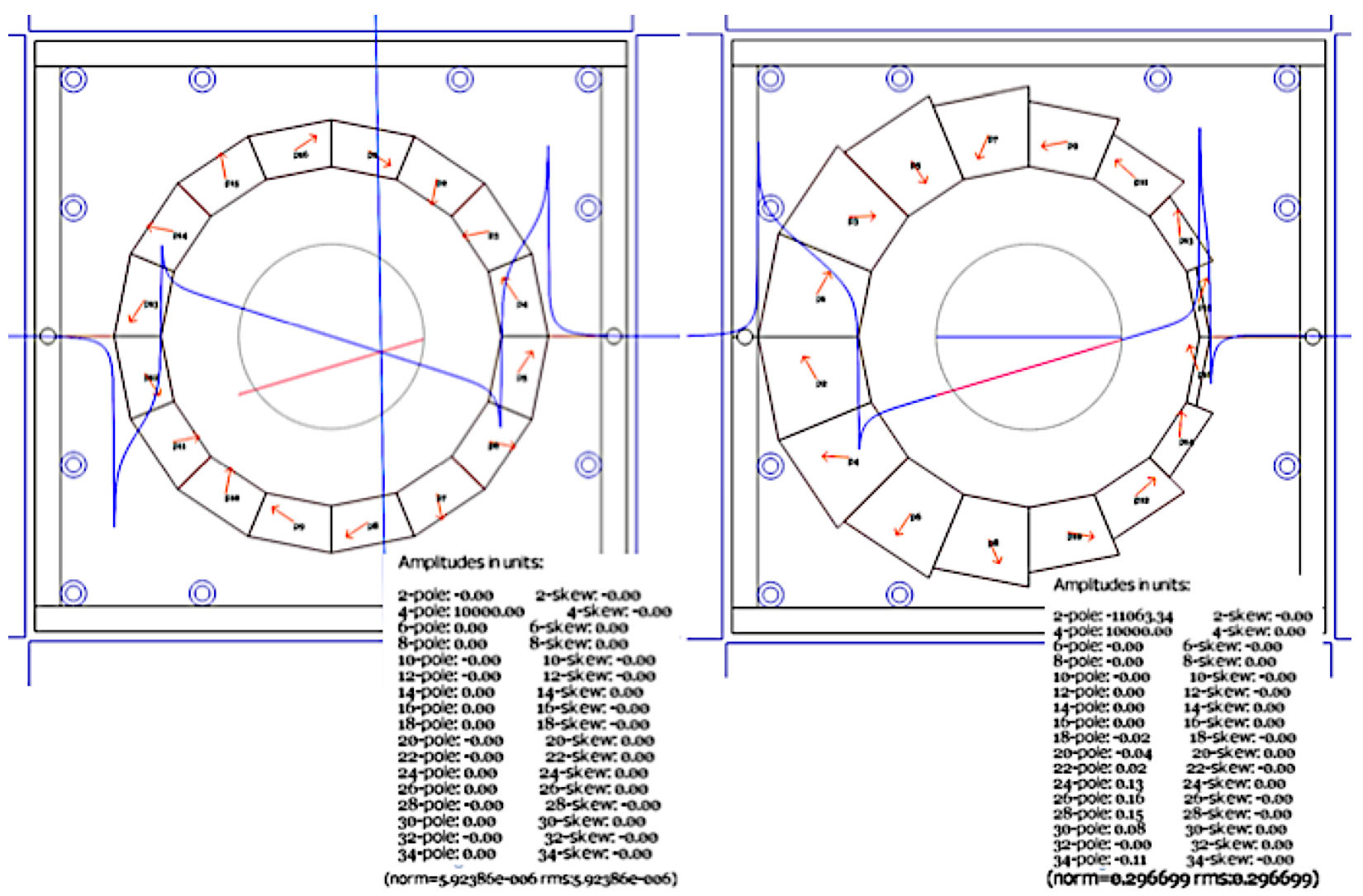

Figure 1: The magnetic field, circles presenting the orbit range (red line) in the focusing Halbach quadrupole (left) and in the combined function Halbach magnet (right) with expected designed multipoles in the lower corners on the right side of each picture. Both inside apertures $(r=4.5 \mathrm{~cm})$ are equal. The outside ends of both permanent magnets fit into the plastic moldings. Both magnet moldings fit into the same aluminum 3/8-inch tick frame with inside size of $13.44 \mathrm{~cm}$. The outside size of the frame is $13.495 \mathrm{~cm}$.

1.1.6 Size and weight are five times less than the $\mathbf{7 . 8}$ and $\mathbf{5 . 8} \mathbf{~ k g}$ for focusing and defocusing magnet respectively, but the iron frame is $\sim 90 \mathrm{~kg} / \mathrm{m}$, while the hybrid magnet or for the whole FFAG arc 780 $\mathrm{kg} / \mathrm{m}$. The CBETA project is a prototype of the Electron Ion Collider (EIC) with the Energy Recovery Linac (ERL) eRHIC. One of the goals of the CBETA is to prove that the FFAG lattice outperforms classical methods of separated arcs (SA). It is important to compare the cost and weight of the SA's made of conventional accelerator technology, requiring significantly smaller number of magnets (it is not strong focusing) and with single beam energy propagation. We estimate $\sim 90 \mathrm{~kg} / \mathrm{meter}$ (TBD) for the Halbach technology versus SA's $250 \mathrm{~kg} / \mathrm{meter}, \sim 780 \mathrm{~kg} /$ meter hybrid (TBD) versus SA's $250 \mathrm{~kg} / \mathrm{meter}$. Another 
important parameter is the arc cross-section, i.e., $20 \times 20 \mathrm{~cm}$ (TBD) Halbach vs. 4 -line $30 \times 30 \mathrm{~cm}, \sim 66 \times 66$ hybrid.

1.1.7 Other possible applications like other ERL's where it would be difficult to convince future ERL's makers that they need 38.5 tons of magnet weight to make an FFAG "simplification". The proton cancer therapy gantries are another possibility. To compare Halbach magnets with correctors with the Hybrid magnets it becomes very clear that Halbach magnets would be the clear choice.

\subsection{Required Goals of the New Halbach Magnet Design}

The CBETA Halbach $150 \mathrm{MeV}$ lattice is aims to be a drop-in replacement for the iron/hybrid magnet lattice as much as possible. Thus, there isn't a "new lattice" for the whole machine. Instead, there is a new FFAG cell involving Halbach magnets that aims to satisfy the following criteria:

- Same magnet lengths

- Same angles between one magnet and the next

- The two above mean machine survey should be identical to the iron lattice

- $Q F$ is a pure quad (central B field zero)

- Maximum ' $x$ ' deviation of any orbit from axis of QF should be minimized, which generally means orbit extremes are equal and opposite in this magnet

- Tunes fill the range $0.03\left(v_{y}\right.$ of $\left.150 \mathrm{MeV}\right)$ to $0.38\left(v_{x}\right.$ of $\left.42 \mathrm{MeV}\right)$

All goals are fulfilled and confirmed but 3D 'OPERA' field design. Three different codes were used to confirm the results from the design: Muon1 code by Stephen Brooks, and ZGOUBI by Francois Meot. The Polymorphic Tracking Code (PTC) confirmed the hard edge design with the fringe fields.

\subsection{Lattice design}

Lattice design for the CBETA has already been based lined. The major parameters of the NS-FFAG cell, presented in the Table 1.3.1, are defined to conform limited properties of the Hybrid-iron magnets:

TABLE: 1.3.1: Hard edge arc cell design parameters.

\begin{tabular}{|c|c|c|}
\hline Injection Total Energy (MeV) & \\
\hline Maximum Total Energy (MeV) & \multicolumn{2}{|c|}{150} \\
\hline Linac Passes & \multirow{2}{*}{\multicolumn{2}{|c|}{$\begin{array}{c}4 \\
5.099439\end{array}$}} \\
\hline Reference Radius (m) & & \\
\hline$L_{D F}(\mathrm{~mm})$ & \multicolumn{2}{|c|}{120} \\
\hline$L_{F D}(\mathrm{~mm})$ & & \\
\hline$\alpha$ & $\mathbf{F}$ & $\mathbf{D}$ \\
\hline$L_{Q \alpha}(\mathrm{mm})$ & 133 & 122 \\
\hline$x_{a}(\mathrm{~mm})$ & -7.182 & +20.132 \\
\hline Gradient $(T / m)$ & +10.621 & -10.017 \\
\hline
\end{tabular}

The lattice design with the Halbach magnets for the CBETA lattice with the maximum energy of $E_{\max }=150$ $\mathrm{MeV}$ follows dimensions defined the in the Table 1.3.1. The hardedge design with the fringe fields is shown in Figure 2. The next step is to design two Halbach magnets using the OPERA 3D program and tune the gradients and fields in a way to obtain as close as possible to the hardedge design. 


\subsubsection{Orbit offsets in the $150 \mathrm{MeV}$ design for Hybrid and Halbach magnet}

The major difference between the two Hybrid-iron-dominated magnets and Halbach magnets are the position of the focusing and defocusing magnets. The Hybrid magnet approach is based on creating the combined function magnet by radially displacing the quadrupoles, while in the Halbach magnet orbits are always placed in the middle of the magnet. The two cases are presented in Figures 2 and 3 , respectively.

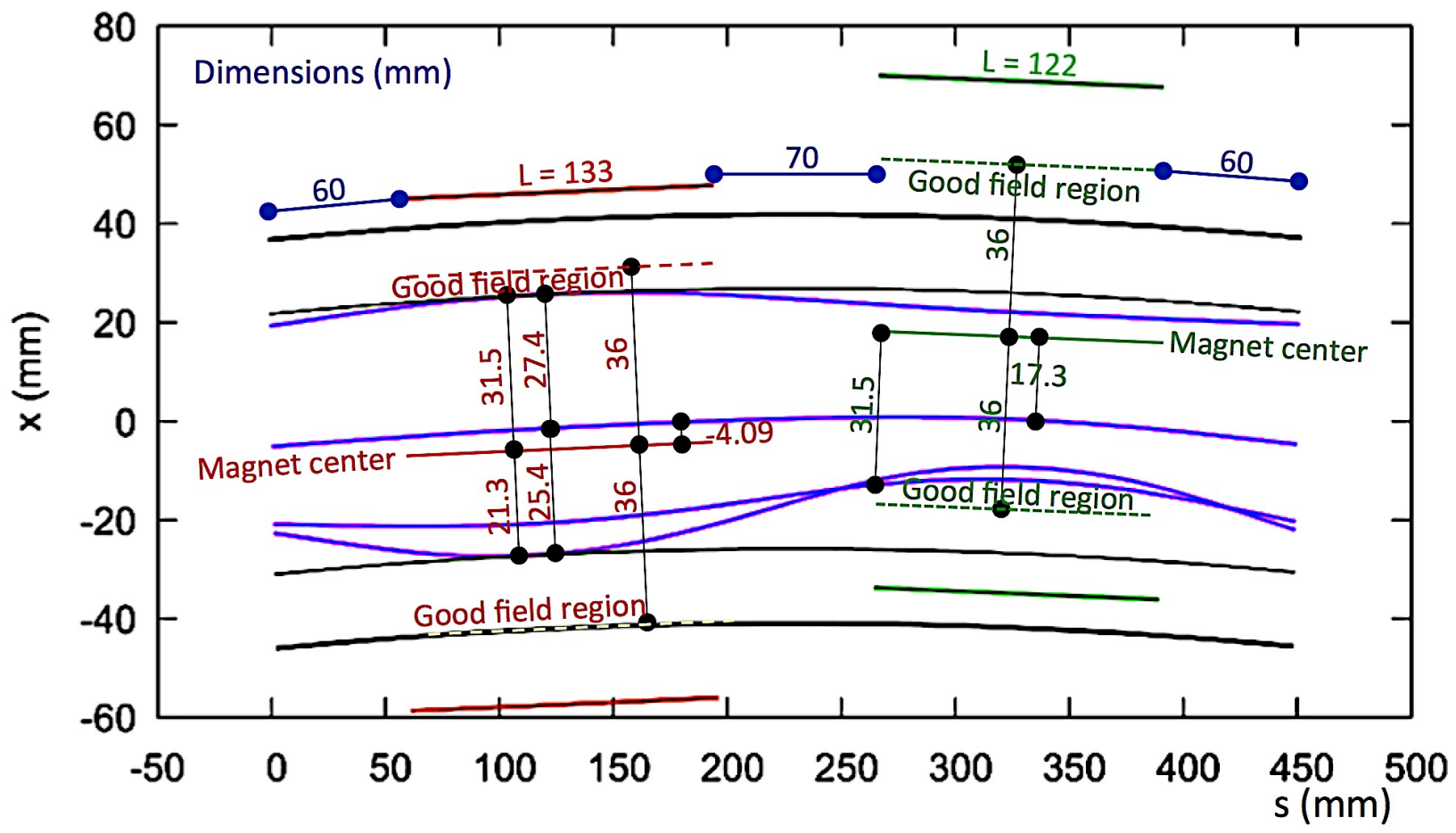

Figure 2: The Hybrid magnets are shown with orbits and centers of the radially misplaced quadrupoles. The misplaced focusing quadrupole magnet is shown on the left side (red lines).

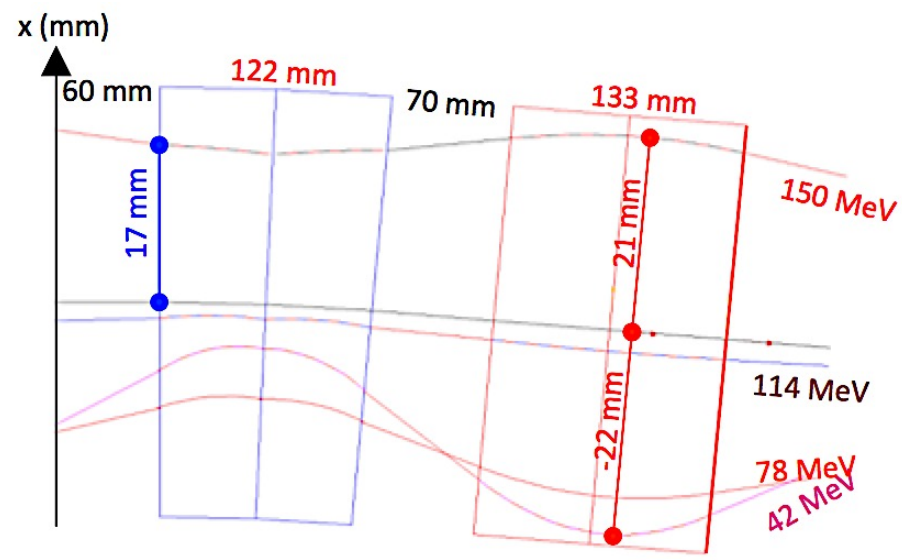

Figure 3: Orbits in the Halbach magnet of the same dimensions and same positions in the same NS-FFAG cell as for the Hybrid magnet cell. The focusing Halbach quadrupole (on the right red color) has a center at the central circular orbit with similar orbit offsets $\pm 22 \mathrm{~mm}$. The inside radius of both Halbach magnets is $4.15 \mathrm{~cm}$.

The focusing hybrid iron quadrupole is misplaced towards inside for only $-4.09 \mathrm{~mm}$, with respect to the central circular orbit $\Delta x=0$. The defocusing quadrupole is misplaced in the opposite direction for +17.3 
$\mathrm{mm}$. The hybrid iron magnet has the largest orbit offsets from the center of both quadrupoles of $\mathbf{3 1 . 5}$ $\mathrm{mm}$. This is the main reason why the good field region for both quadrupoles is $36 \mathrm{~mm}$ and that raises the magnet size with the outside dimension of $66 \mathrm{~cm}$. The internal dimensions of the beam pipe placed between the iron poles are $84 \times 24 \mathrm{~mm}$.

Another way to compare the two cases is presented in Figure 7.

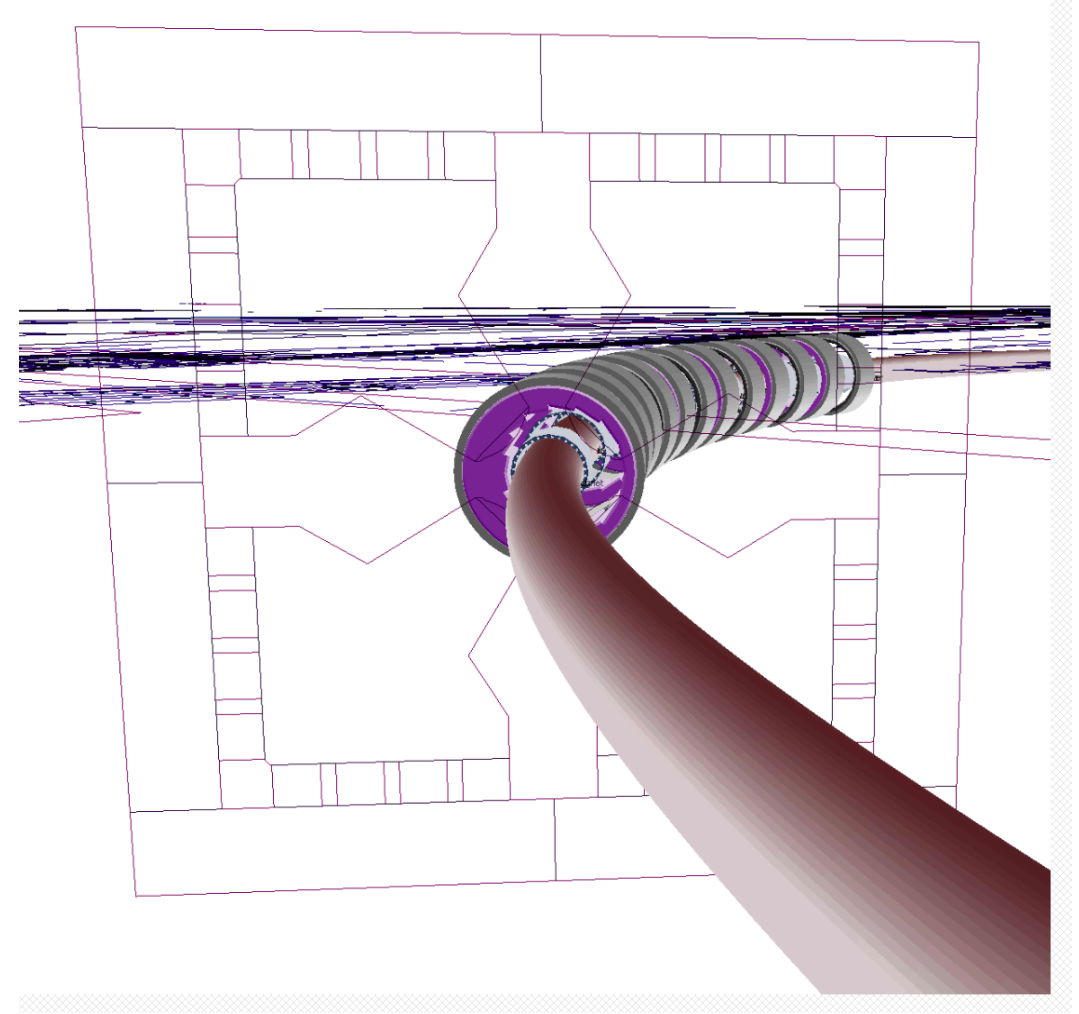

Figure 4: Both magnet designs presented in the same frame for the maximum energy $E_{\max }=150 \mathrm{MeV}$.

\subsubsection{Halbach Magnet Design with "OPERA 3D" and Tests from Tracking}

Stephen Books using the Muon1 program obtained the Halbach magnet design shown in Fig.1. He defines the permanent magnet blocks and provides the information for "OPERA 3D" calculation by Nick Tsoupas, they are shown in Fig. 5 and details for the future supplier. From the input the OPERA 3D produces two Halbach magnets as shown in Fig. 5. The 3D field maps are used to track electrons with either Muon1 or ZGOUBI and the real lattice parameters like tunes, twiss functions, orbit offsets, dispersion are to be compared to the previous hardedge design. This process requires couple of iterations as the non-linear fields between the magnets introduce differences to be corrected by variation of the gradients. The positions and lengths of the magnets were kept fixed following the previously fixed baseline lattice design. The results for the layout and orbits, obtained by Muon1 by Stephen Brooks, are shown are Fig. 5. The magnetic fields maps were read by the ZGOUBI program and 1000 electrons tracked through the magnets. The magnetic field and orbits from the 3D field are shown in Fig. 7, the dynamical aperture admittance and the time of flight are shown in Figure 8. 

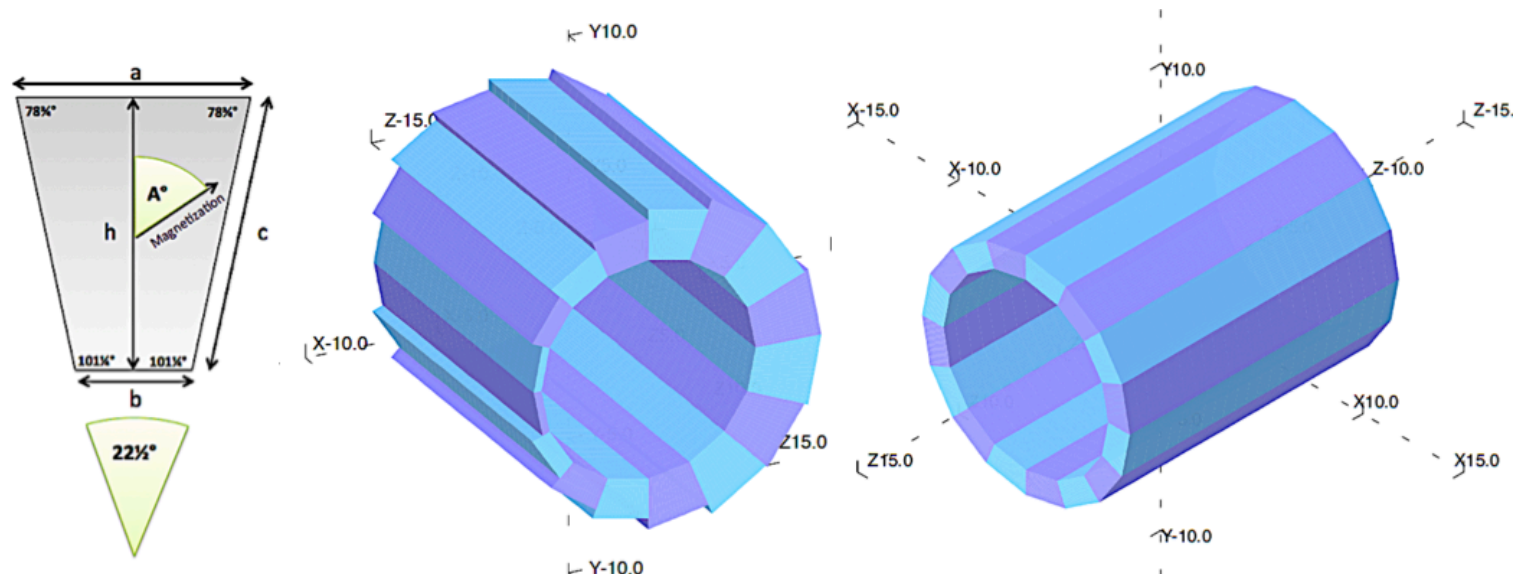

Figure 5: Specification of the Halbach permanent magnet blocks for the OPERA program, as well as for the suppliers, and the OPERA 3D picture of the defocusing (left) and focusing (right) Halbach magnets (by Nick Tsoupas).
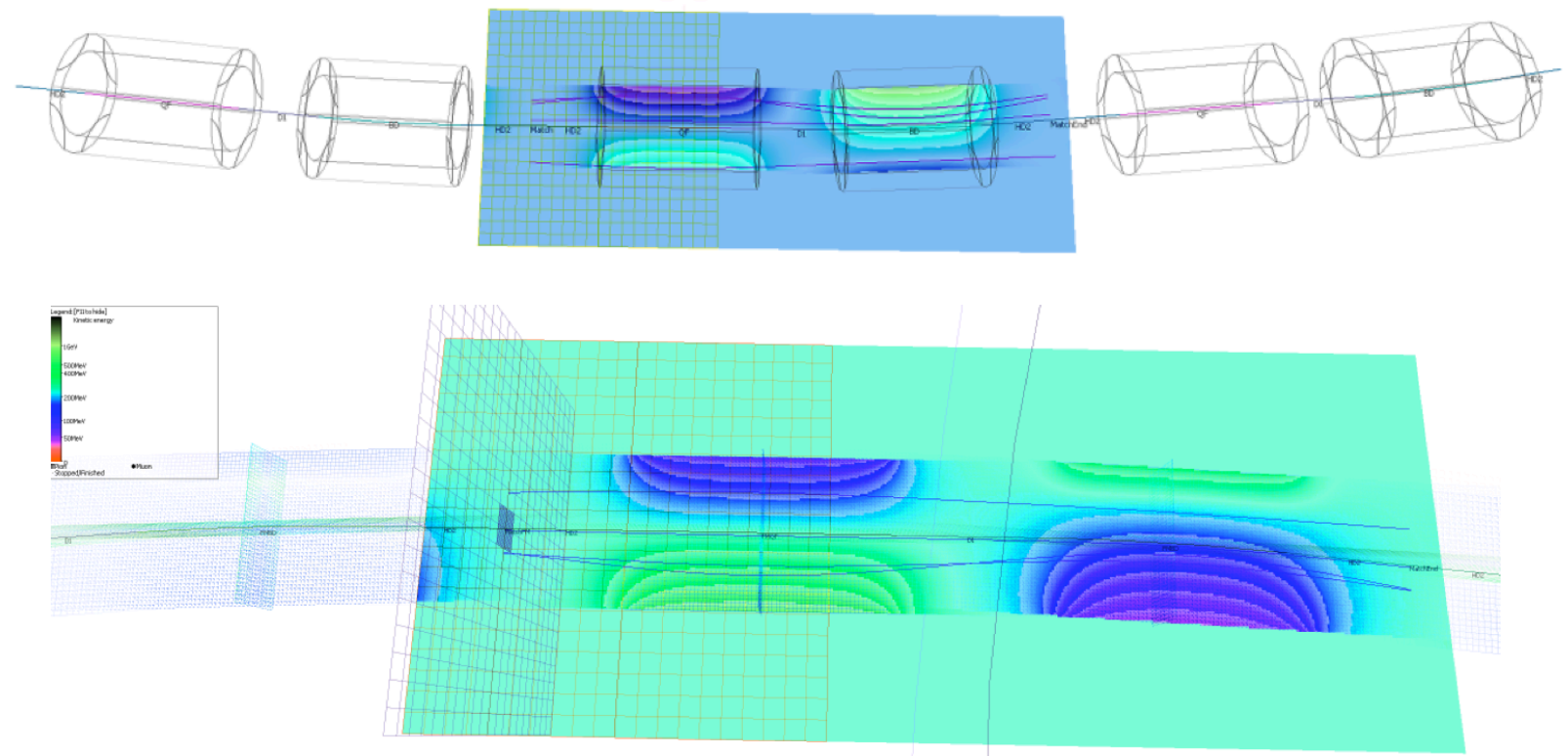

Figure 6: Muon1 orbits tracked through the 3D OPERA fields in couple of cells (above) and in a single NSFFAG cell (bellow) by Stephen Brooks.

Field along orbits

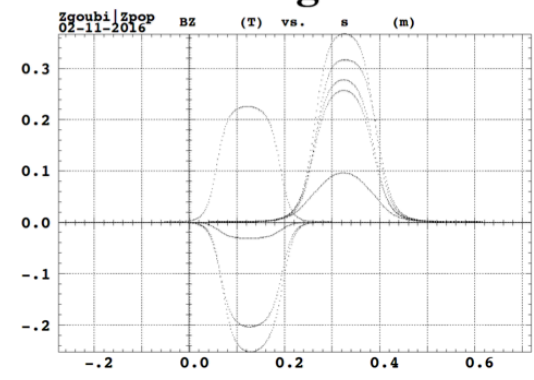

\section{Orbits in QF}

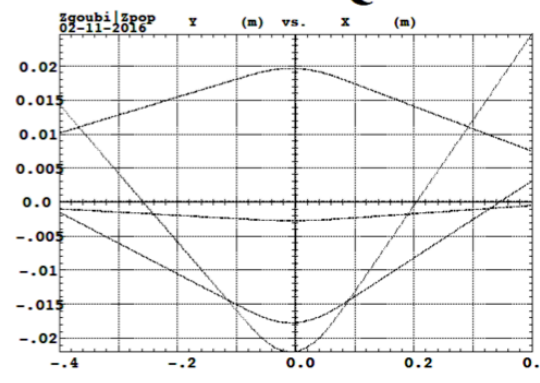

Orbits in BD

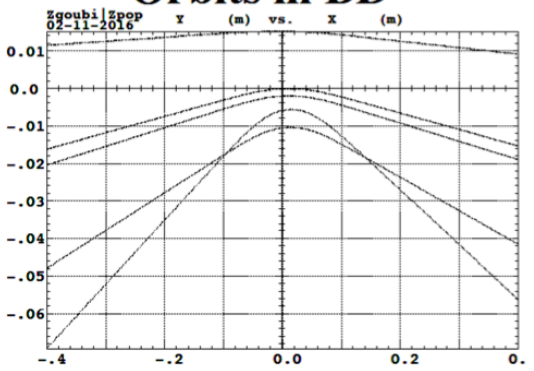

Figure 7: ZBOUBI magnetic fields obtained by OPERA with orbits in the focusing and defocusing 150 MeV Halbach magnets from Francois Meot. 
The horizontal and vertical chromaticities $\xi_{x}$ and $\xi_{y}$, for the hard edge model and obtained by tracking 1000 electrons are shown in Figure 9.
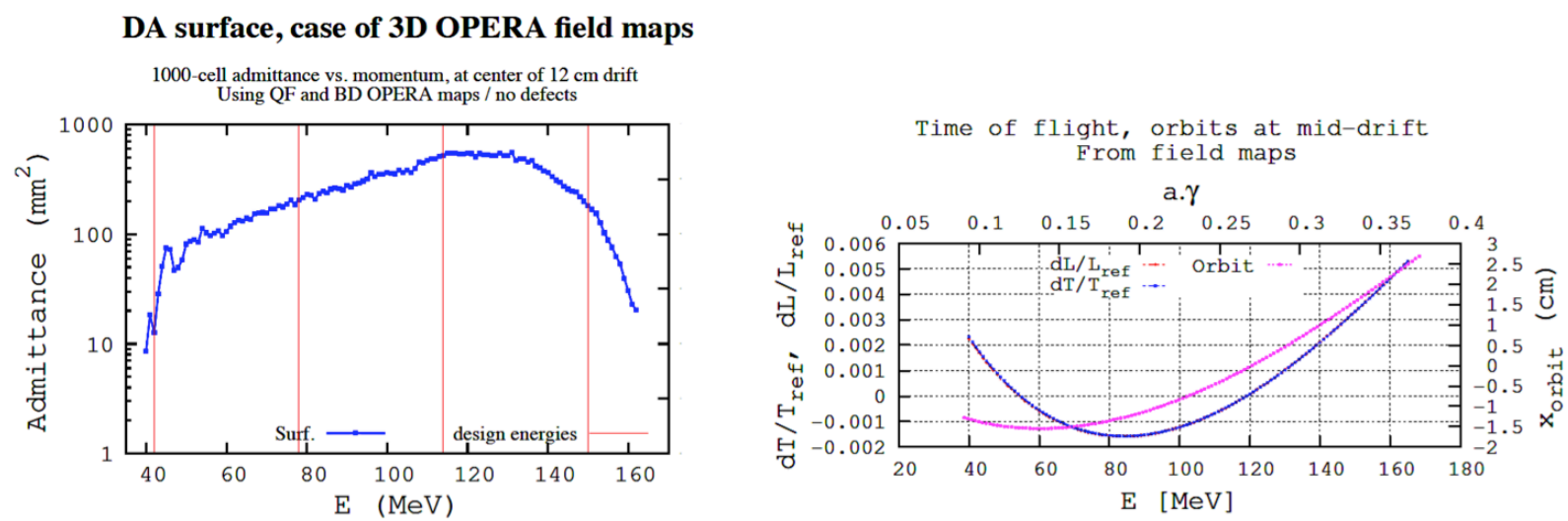

Figure 8: Results from the ZGOUBI program from tracking through the 3D field maps from OPERA 3D of the Dynamical admittance in $\mathrm{mm}^{2}$ and the time of flight for different energies by Francois Meot.

\section{- Hard edge model}

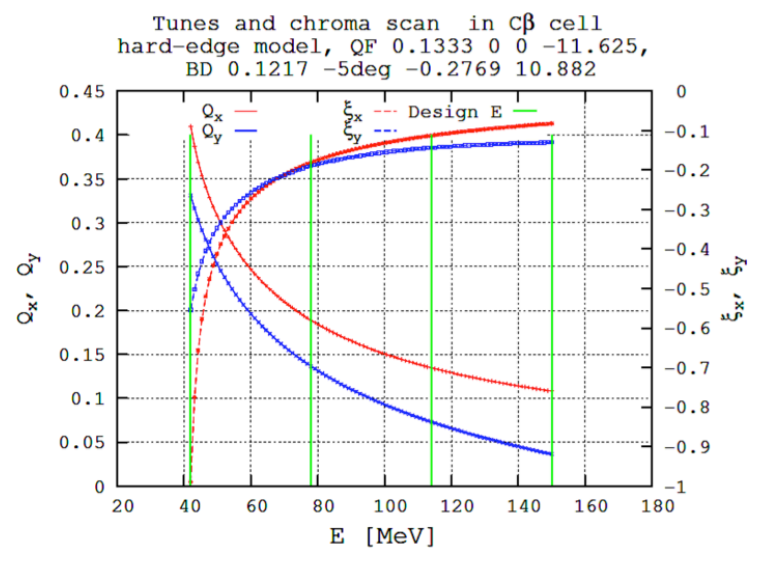

- Field map model

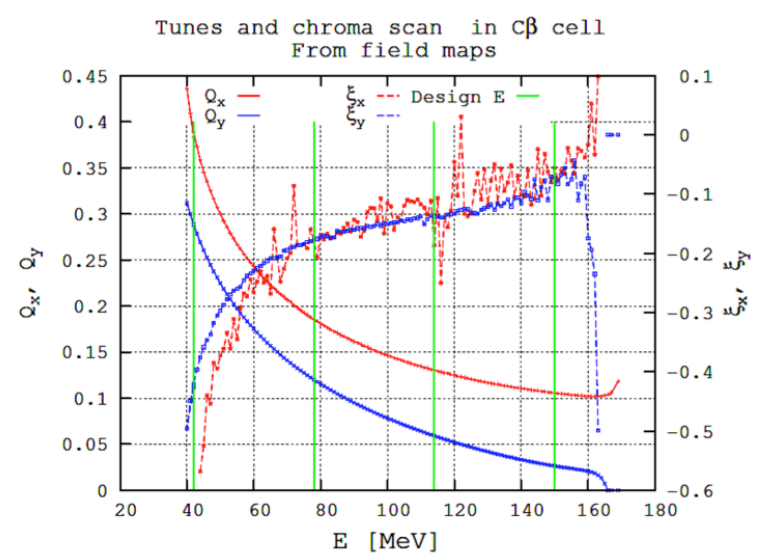

Figure 9: Chromaticities from the hard edge model and from the tracking 1000 particles for different energies by ZGOUBI from Francois Meot, through the 3D field maps obtained by OPERA 3D by Nick Tsoupas.

The "Muon1" program (by Stephen Brooks) and the ZGOUBI program by Francois Meot) showed an excellent agreement for both hard edges as well as from the 3D magnetic field tracking.

\subsection{Overall Assemblies and Quantity}

There are two types of magnet assembly, QF and BD. These share a common outer frame and also include the same window frame corrector around them. The differences are only (a) the thickness of the NdFeB permanent magnet wedges, (b) the shape of the 3D printed mold fitting around the wedges inside the aluminum holder and (c) the length of the overall magnet. All assemblies are splittable in order to fit the vacuum pipe through them. 
Table 1.4.1: Numbers of assemblies and lengths

\begin{tabular}{|c|c|c|c|}
\hline & Number in design (CDR) & $\begin{array}{c}\text { Number in magnet costing } \\
\text { (includes some spares) }\end{array}$ & Length (mm) \\
\hline QF & 106 & 110 & 133.3 \\
\hline BD & 106 & 110 & 121.7 \\
\hline Total & 213 & 220 & \\
\hline
\end{tabular}

\subsection{Assembly procedure}

The production of the necessary parts for the Halbach magnets is based on our experience with the previous $250 \mathrm{MeV}$ Halbach 12 prototype magnets as shown in Figure 10.

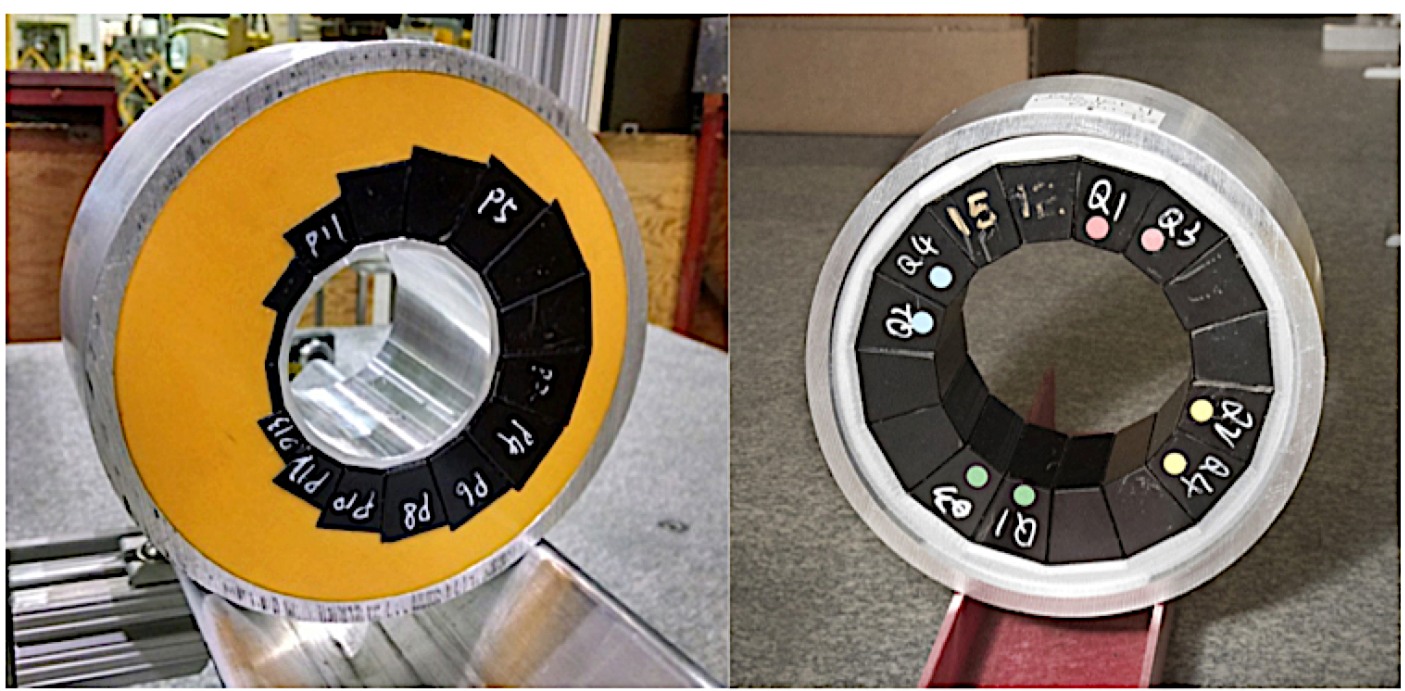

Figure 10: Defocusing (left) and focusing (right) Halbach prototype magnets for CBETA $E_{\max }=250 \mathrm{MeV}$.

As previously the production starts with building the plastic molds by the 3D printers to get connected with the permanent magnetic prisms (ordered for the manufacturer "Alstar Magnetics", previously used for the $250 \mathrm{MeV}$ twelve prototypes). The second part in production is connection of the of $3 / 8^{\prime \prime}$ aluminum plates, put together under a $90^{\circ}$ angle, as shown in the upper left side of the Figure 11. The three aluminum plates are sequentially glued to the upper or lower plastic mold and connected to the right angle with the stainless steel bolts size 10 as shown in Figure 11. The plastic molds are glued into the aluminum plates with the 3M 5200 marine adhesive sealant (used to extremely hard bond fiberglass deck to hull, wood to fiberglass, motors on fiberglass transom, stern joints etc.). The sealant has already been tested with gluing the permanent magnetic prisms on the plastic mold made by the 3D printer. Additional molds are necessary in assembly procedure as it was found out during the previous prototype production: each pair (in the case of the combined function magnet) of permanent magnetic prism has copies made of plastic molds produced by the 3D printers (as shown in Figure 11): A necessary mold in the middle of the magnet is also made of plastic and two halves of the magnet (lower and upper) are first made of plastic molds. The next step is a creation of the alignment holes. A drilling of the alignment notches into the aluminum $3 / 8^{\prime \prime}$ frame at the middle of the assembly is possible as the upper and lower parts are tide together in a very precise fit, as shown in the Figure 12. 


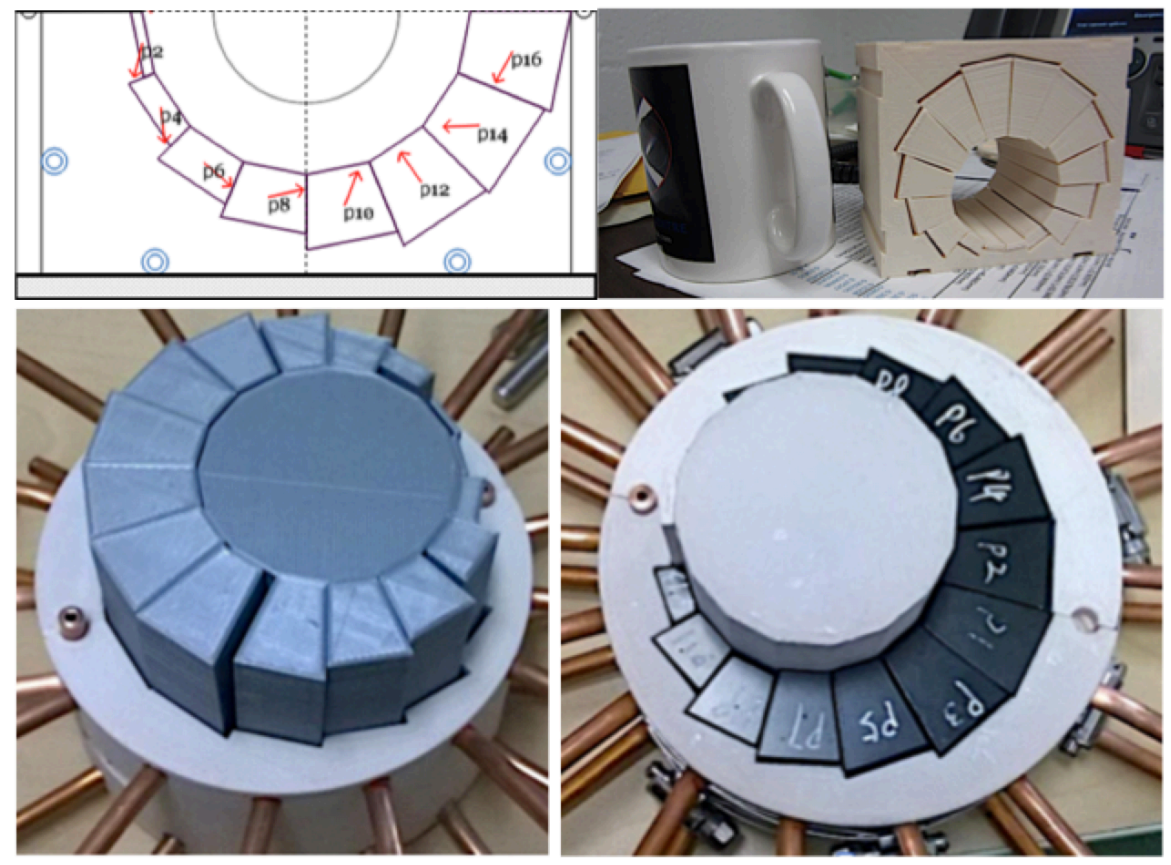

Figure 11: Assembly procedure and necessary plastic molds obtained by the 3D printer to allow assembly of the permanent magnet material. The plastic molds, one by one, are replaced by the permanent magnet blocks (as shown in the lower right part).

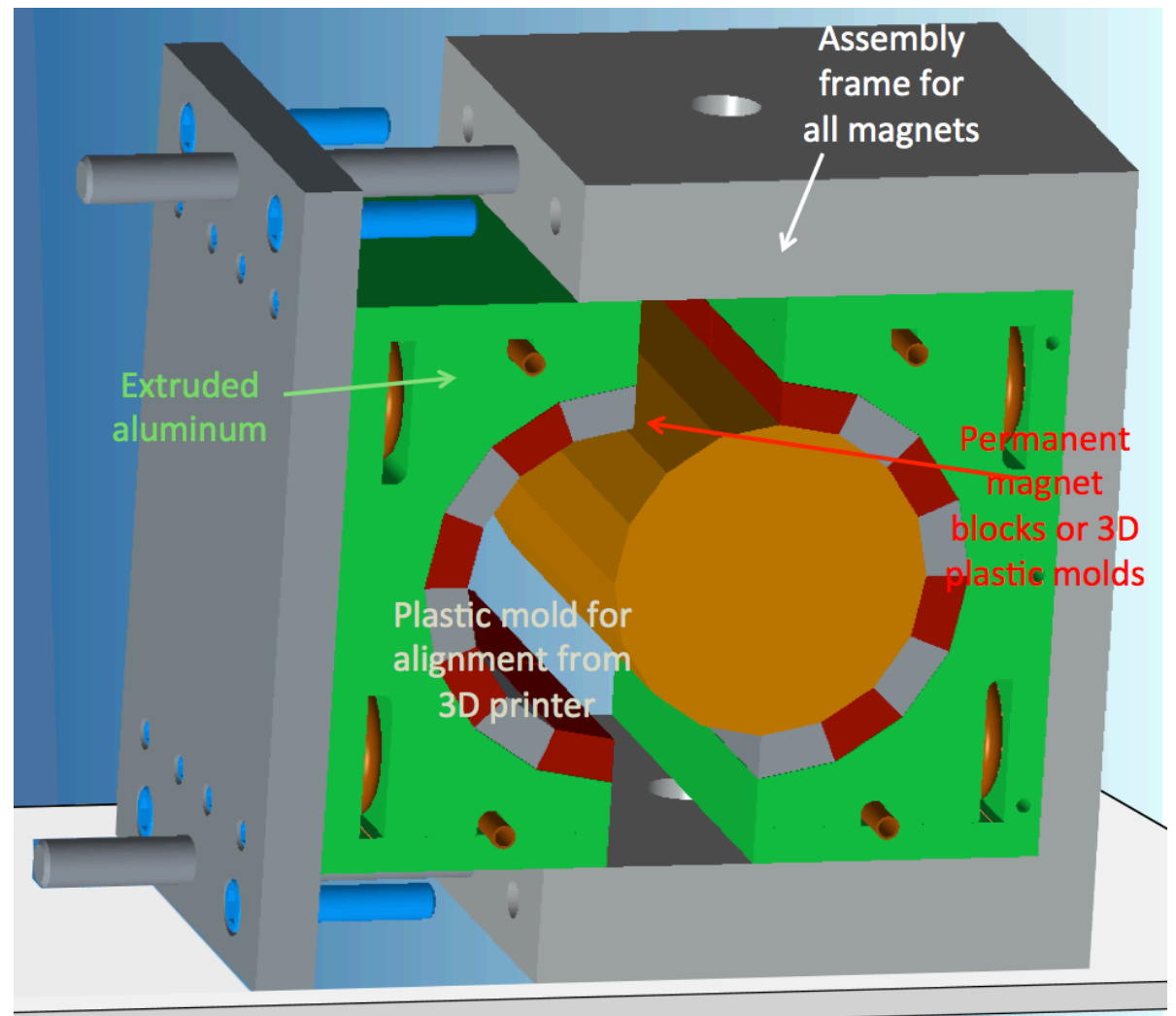

Figure 12: An aluminum frame-fixture to be used for all Halbach magnets with left and right parts with just glued in plastic molds with copper pipes and permanent magnet blocks. The two assemblies are firmly tide together and left for 8 hours necessary for the 3M 5200 glue to cure. 


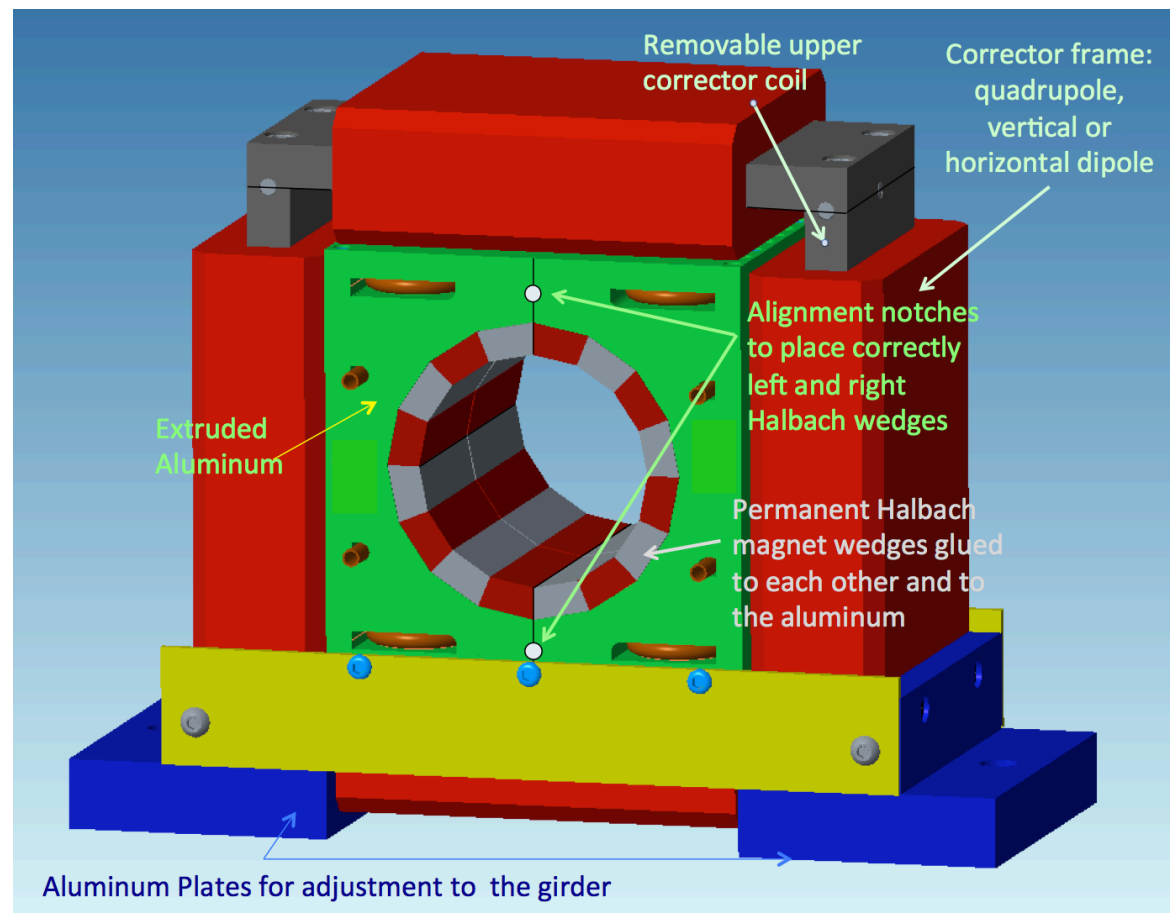

Figure 13: The Halbach defocusing magnet assembled together with the correction magnet frame.

Disassembly procedure requires removal of the upper corrector coil as shown in Figure 13.

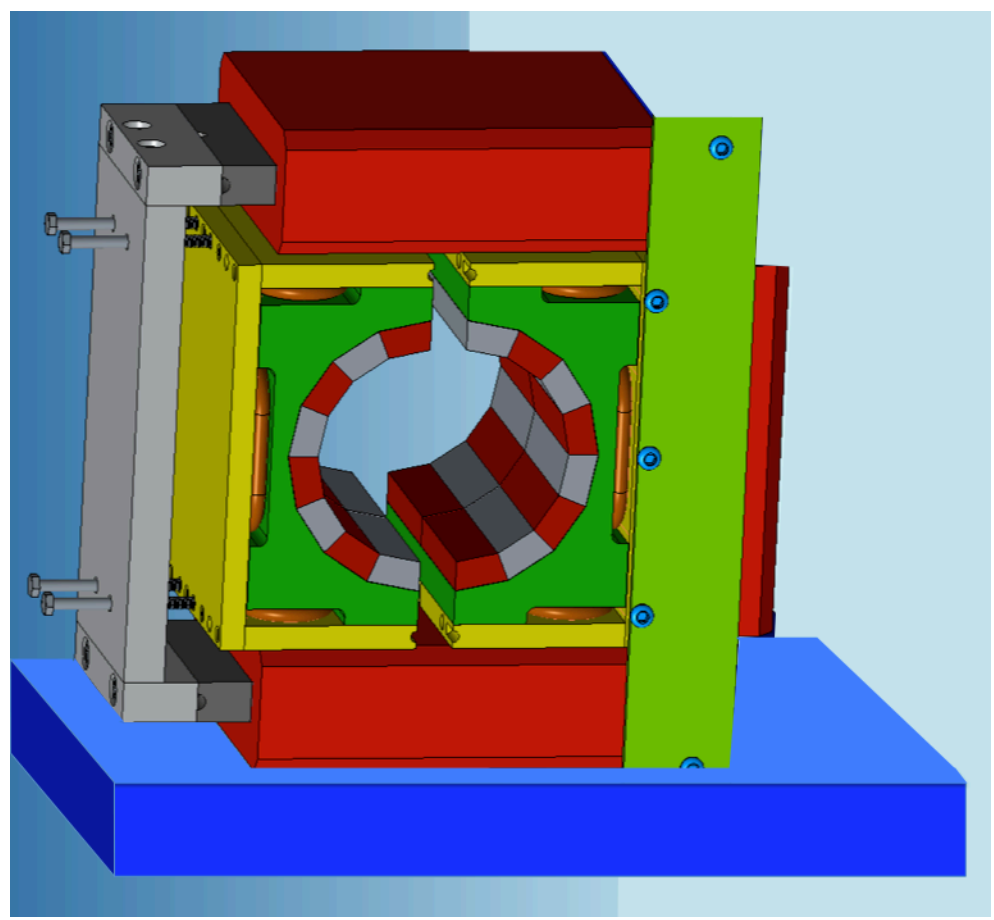

Figure 14: Disassembly procedure starts with removal of the right corrector coil and placement of the left fixtureframe (gray color). Four bolts (shown on the upper additional frame) have enough strength to overcome the attraction force between the permanent magnet blocks of the middle plane. 


\subsection{Cross-Sections with Dimensions}

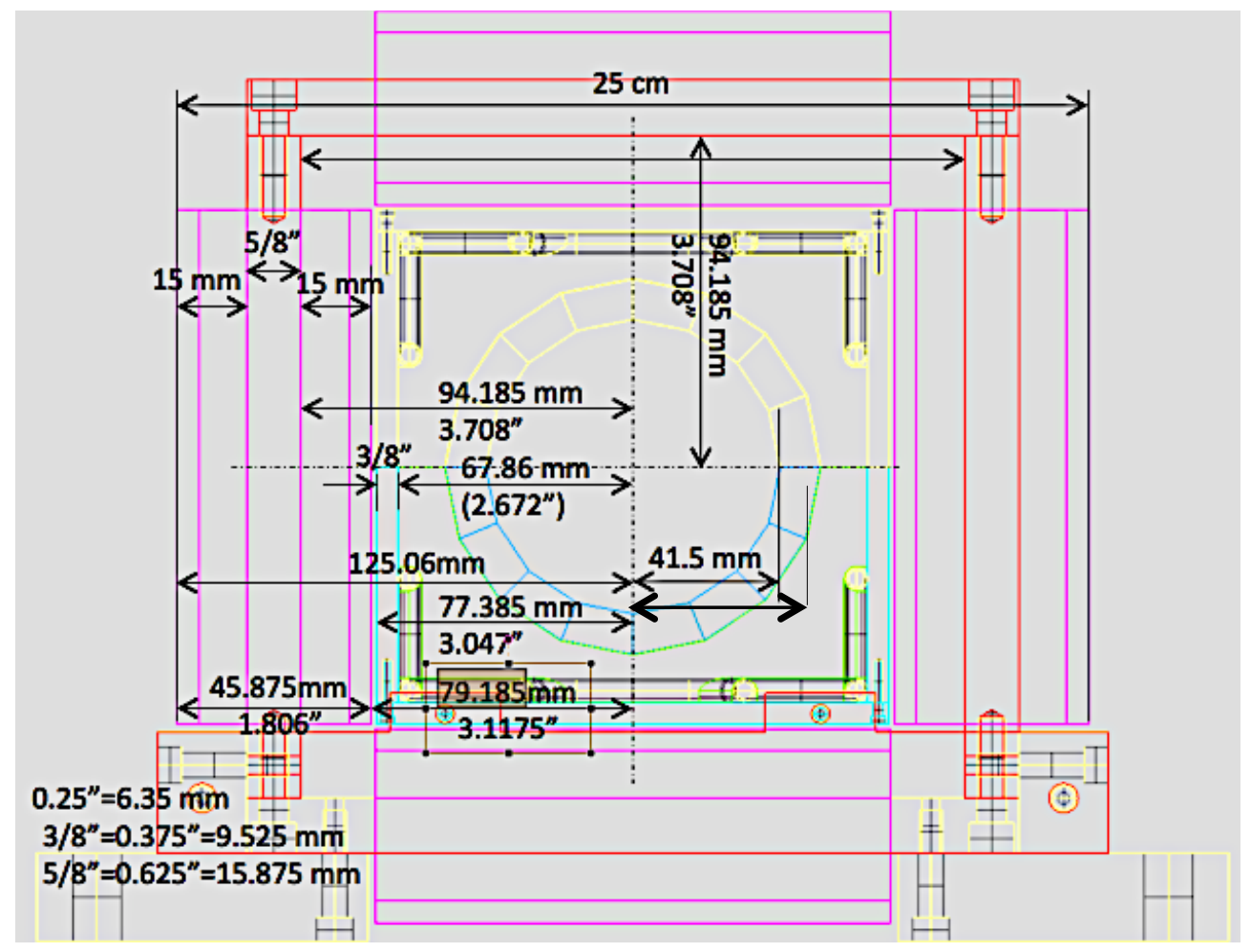

Figure 15: QF magnet cross-section and dimensions: Aluminum frame $3 / 8$ " $\mathrm{cm}$, iron corrector frame $5 / 8$, aluminum frame distance from the center of Halbach quadrupole to the inside of the frame $67.86 \mathrm{~mm}$. A distance between the aluminum frame and the cured copper coil $1.8 \mathrm{~mm}$, the width of the copper coils is $15 \mathrm{~mm}$. The total dimension from end of the copper coil to the other side end is $23.2975 \mathrm{~cm}$.

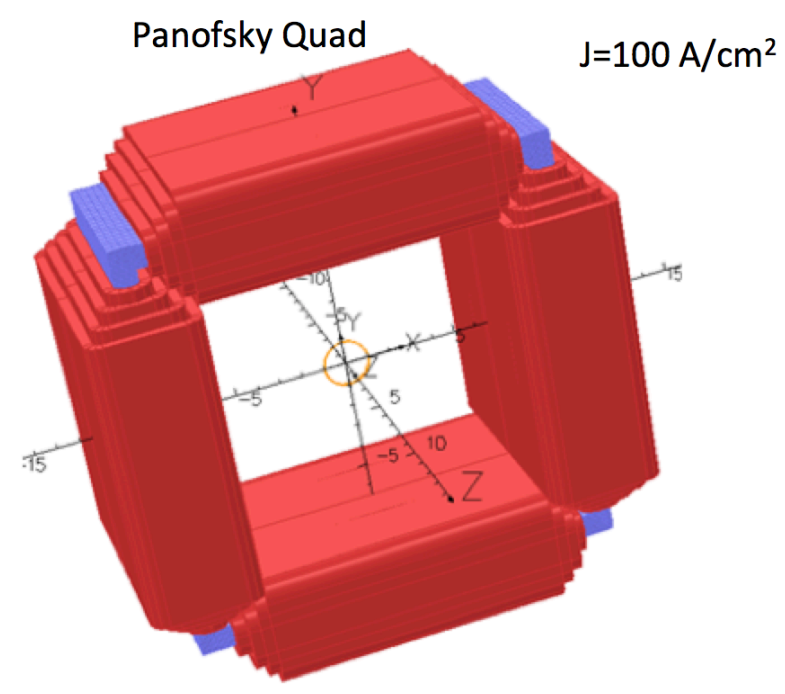

Figure 16: Window frame corrector model in OPERA-3D by Nick Tsoupas.

The window frame iron is 5/8" thick and the coils are $15 \mathrm{~mm}$ thick on each side. An OPERA-3D model of the corrector is shown in figure 4. The window frame iron is shortened in the longitudinal axis so the coils do not "overhang" the nominal magnet length. 
Table 1.6.1: Parts List (excluding pins, screws etc.)

\begin{tabular}{|l|l|l|l|}
\hline Part & Material & Number per magnet & Total number \\
\hline $\begin{array}{l}\text { Permanent magnet } \\
\text { wedges }\end{array}$ & NdFeB & 32 (2 layers of 16) & 7040 \\
\hline Shim holder halves & 3D printed plastic & 2 & 440 \\
\hline Shimming wires & $1006 / 1008$ steel wire & 32 & 7040 \\
\hline Molds to hold wedges & 3D printed plastic & 2 & 440 \\
\hline Copper cooling pipes & Bent 1/4" copper tube & 2 & 440 \\
\hline Halbach casing & 1/4" thick Al plate & 6 & 1320 \\
\hline $\begin{array}{l}\text { Halbach-to-window } \\
\text { frame attachment } \\
\text { plates }\end{array}$ & Aluminum plate & 4 & 880 \\
\hline Window frame iron & $15 m m$ thick 1006 iron & 4 & 880 \\
\hline Window frame coils & Cu potted in epoxy & 4 & 880 \\
\hline
\end{tabular}

Table 1.6.2: Assembly Methods

\begin{tabular}{|l|l|l|}
\hline Part A & Part B & Attachment method \\
\hline Permanent magnet wedges & Mold to hold wedges & $\begin{array}{l}\text { Glue, while hammering blocks } \\
\text { into plastic mold }\end{array}$ \\
\hline Permanent magnet wedges & Permanent magnet wedges & Glue \\
\hline Permanent magnet wedges & Shim holder halves & $\begin{array}{l}\text { Glue before commencing } \\
\text { shimming/rotating coil }\end{array}$ \\
\hline Shimming wires & Shim holder halves & $\begin{array}{l}\text { They stick there by magnetic } \\
\text { force, helped by sockets in the } \\
\text { shim holder, but can glue or } \\
\text { epoxy in place when done }\end{array}$ \\
\hline Molds to hold wedges & Halbach casing (halves) & Glue, should also be good fit \\
\hline Halbach casing (Al plates) & Halbach casing (Al plates) & $\begin{array}{l}\text { Screws attaching two plates at } \\
\text { 90 degrees }\end{array}$ \\
\hline Halbach casing (upper half) & Halbach casing (lower half) & $\begin{array}{l}\text { Pins in drilled holes to ensure } \\
\text { accurate, repeatable alignment }\end{array}$ \\
\hline Halbach casing (lower half) & Window frame iron (lower half) & $\begin{array}{l}\text { Via the Halbach-to-window } \\
\text { frame attachment plates, using } \\
\text { screws or bolts }\end{array}$ \\
\hline Window frame coils & Window frame iron & Manufacturer's choice \\
\hline Window frame iron (top/lid) & Window frame iron (lower part) & $\begin{array}{l}\text { Manufacturer's choice, } \\
\text { probably screws }\end{array}$ \\
\hline Window frame iron (lower part) & Girder & 6-axis adjustable mount \\
\hline
\end{tabular}




\subsection{Shimming Method}

1. Assemble whole magnet including corrector iron

2. Glue shim holder halves into bore 3 . 1st rotating coil measurement

4. Run program that outputs wire sizes/lengths (fast)

5. Cut wires to length and insert in shim holder (1hr)

6. 2 nd rotating coil measurement

a. Include survey of coil axis to magnet fiducials this time

7. If harmonics are low enough finish, otherwise re-shim for another iteration (go to step 4). Typically only 1 or 2 iterations ( 2 or 3 coil measurements total) are required.

\subsection{Vacuum Round Pipe Design (Four Button BPM's)}

The vacuum system for the Halbach magnet had previously been design with a round pipe, as this does not represent a problem. The inner size of both focusing and defocusing magnets are the same $r=4.15$ $\mathrm{cm}$. The outside dimension of the aluminum frame surrounding the plastic mold made by the 3D printer is $3^{\prime \prime}$. The corrector assembly has a total of $25 \mathrm{~cm}$ outside dimension. Advantages of the round pipe with respect to the flat pipe are multiple: necessary and complicated transitions from the flat chamber to the round one to allow adjustment in the horizontal plane is not needed in the case of the round pipe as shown in Fig. 17. The drifts (designed dimensions are 7 and $12 \mathrm{~cm}$ ) are not reduced due the corrector coils ends as the correctors are placed inside of the Hybrid iron magnets. Assembly of the magnets is dramatically simplified, as it is not necessary to use the cranes for each magnet. There is an easy access to the magnets as the outside distance from the corrector frame to the vacuum pipe is only $12.5 \mathrm{~cm}$, while in the case of the hybrid iron magnet a distance is $\approx 60 \mathrm{~cm}$. The major advantage comes from the 4 button BPMs instead of 6 buttons, not only the installation but also the number of electronic boards is reduced to half and cost is reduced from $\$ 660,000$ to $\$ 330,000$.

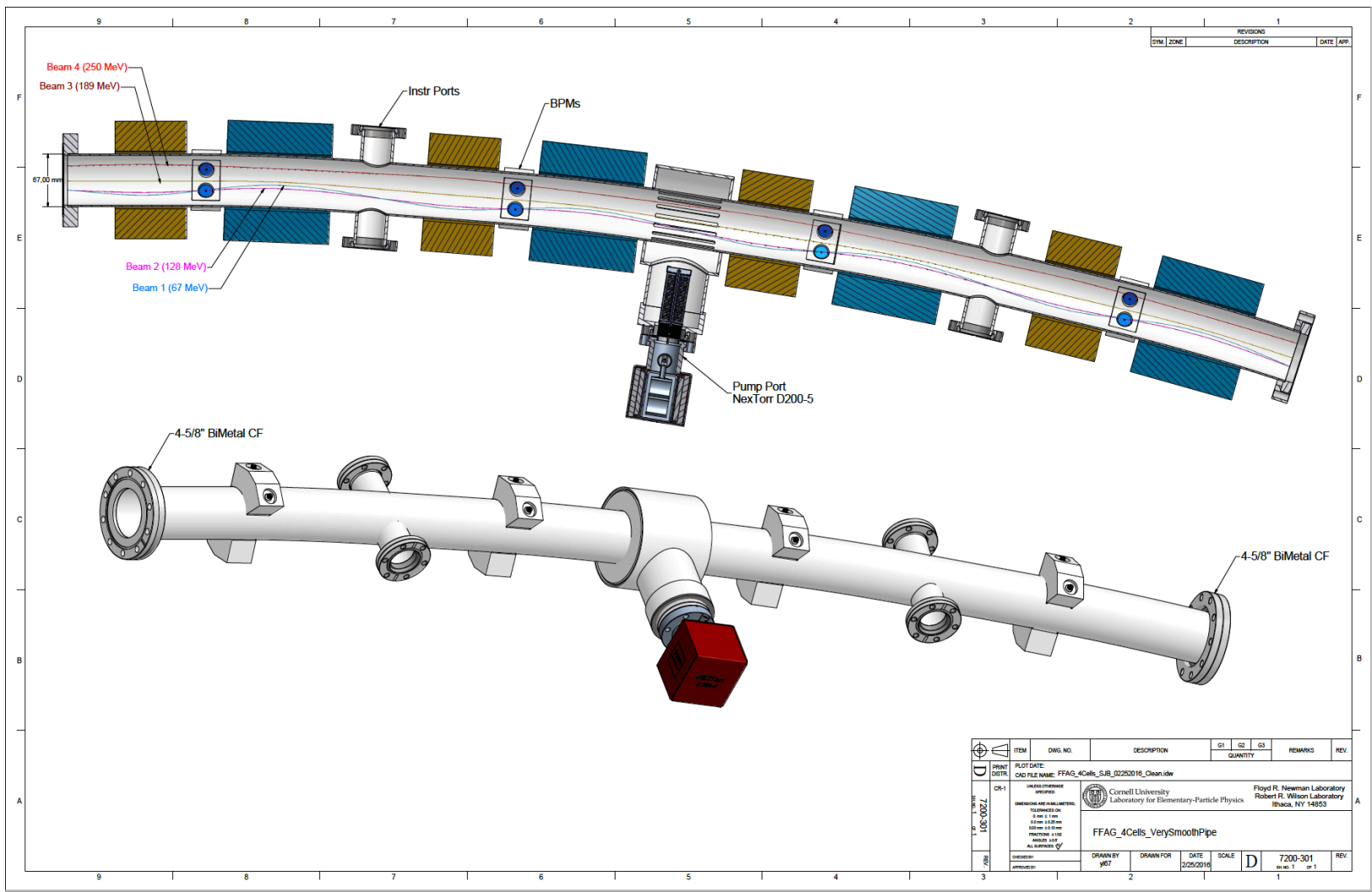

Figure 17: A girder vacuum system design. 


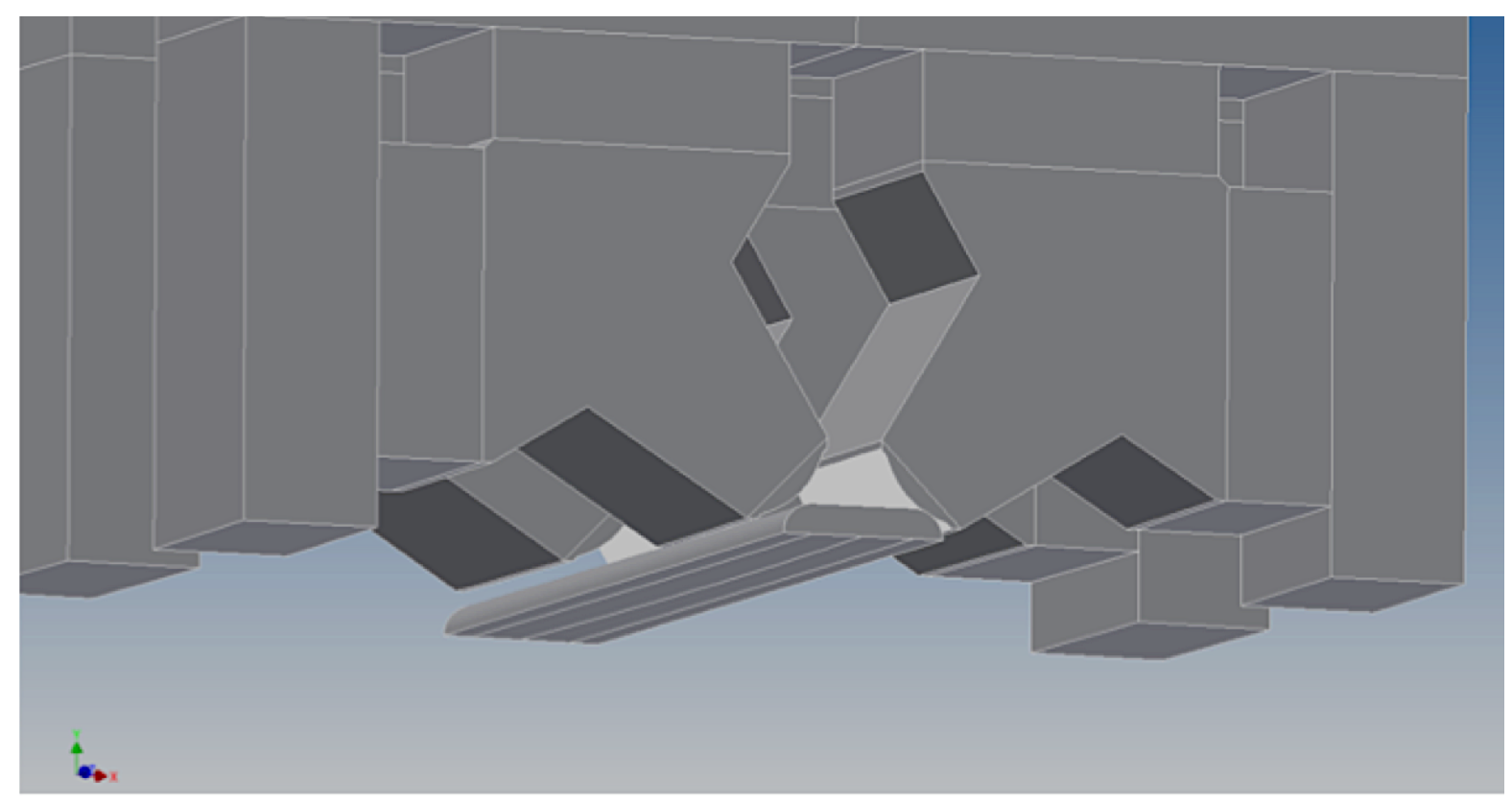

Figure 18: Flat beam vacuum pipe placement in the Hybrid-iron magnet.

\subsection{Results from Prototype Halbach Magnets (250 MeV)}

The CBETA project has requirements for an FFAG arc with radius no more than 5 meters that bends electron energies of up to $250 \mathrm{MeV}$.

The maximum radius is dictated by the available space in the LOE hall at Cornell University, so as to leave space for shielding blocks and movement of people, while not penetrating outside the overall walls of the building or into areas used for other equipment. The building itself is authorized for radiation sources like accelerators, while the outside is not, so the hall footprint is a fairly hard limit that would require permits and extensive construction work to circumvent.

The energy of $250 \mathrm{MeV}$ is designed to fit in with the FFAG $4 \mathrm{x}$ momentum range, the Cornell cryomodule energy gain and potential physics applications of the circulating beam. The FFAG momentum range is designed to bracket recent eRHIC designs that used $3 x$ and $4 x$ ranges in their FFAGs and is a significant advancement over the $2 x$ range of EMMA. Although initially the cryomodule was specified for " $100 \mathrm{MeV}$ ", this is an absolute maximum and running with an FFAG requires a specific energy that has a high degree of confidence of being achieved. The current design therefore calls for $61 \mathrm{MeV}$ energy gain per turn, which has been verified as possible in tests even with one cavity not running. With a $6 \mathrm{MeV}$ injector this makes for a maximum energy in the FFAG of $6 \mathrm{MeV}+4 * 61 \mathrm{MeV}=250 \mathrm{MeV}$. The physics applications and requirements are available from references in section 2 of http://arxiv.org/pdf/1504.00588v1.pdf. They prefer a higher energy, up to 300MeV ideally, although $250 \mathrm{MeV}$ is an acceptable compromise.

Given these parameters, the FFAG lattice puts fairly challenging requirements on the magnets in the arc. FFAGs are machines, which intrinsically require a high gradient in the magnets, so the varying field can 
transport the very wide range of energies. This leads to high gradient magnets with short focusing periods. In CBETA, the small arc radius also shortens the magnets and increases the required dipole and quadrupole fields, both in dipole and quadrupole, although it should be noted in FFAGs the "quadrupole component" is often the main bending field since the electron bunches are circulating off-axis. Forcing the focusing periods to be longer would increase the separation of the different energy beams roughly by the square of the focusing length, so the magnets would get wider faster than they get longer, actually making the aspect ratio unfavorable. Shortening the periods would lead to theoretically narrow, but extremely high-gradient magnets that would not be wide enough to accommodate the vacuum chamber. To take account of this, an additional design constraint was added that the distance from the physical magnet pole to the ideal centroid of any of the beam orbits must be at least $17 \mathrm{~mm}$. This consists partly of $12 \mathrm{~mm}$ clearance between the beam center and the inner wall of the vacuum chamber, to fit the beam size, possible halo, allow for steering errors and reduce resistive wall effects from going too close to the wall. A further $5 \mathrm{~mm}$ allows for the thickness of the vacuum chamber, which can be up to $3 \mathrm{~mm}$ for extruded parts, some mechanical clearance, plus $1 \mathrm{~mm}$ for shims to be placed inside the magnet bore if necessary. In an initial round of studies, a magnet design that uses iron poles driven by permanent magnets was found to be difficult to satisfy the constraints of the FFAG design. So the Halbach design with bare blocks of magnet arranged around the aperture was investigated as an alternative, because Halbach dipoles are known for being able to achieve very high fields. This yielded designs compatible with the FFAG lattice, subject to a few details later in this report. It should be noted that while the dipole achievable in the Halbach magnet is theoretically unlimited (the magnet outer radius expands exponentially with field), the quadrupole pole-tip field is not. There is a hard limit of gradient*bore for any given material grade, which the FFAG lattice has to avoid. It should also be noted that more recent co-optimization of the iron-dominated magnet design with the FFAG lattice is showing that the iron pole design may be possible after all, avoiding some of the complications of the Halbach arrangement (this work is currently being conducted by Holger Witte and J. Scott Berg).

The most recent CBETA FFAG arc lattice using the Halbach type magnets is called Cell_Smoothpipe_2016-02-04. The magnet requirements for this cell, which consists of one focusing (QF) and one defocussing and bending (BD) magnet, are shown in the Table 1 below.

Table 1.9.1: Magnet parameters for the prototype Halbach magnets for CBETA with $E_{\max }=250 \mathrm{MeV}$

\begin{tabular}{|c|c|c|}
\hline Parameter & QF magnet & BD magnet \\
\hline Length & $96.3 \mathrm{~mm}$ & $126.4 \mathrm{~mm}$ \\
\hline Gradient & $-28.80 \mathrm{~T} / \mathrm{m}$ & $19.19 \mathrm{~T} / \mathrm{m}$ \\
\hline Dipole component at center & 0 & $-0.2680 \mathrm{~T}$ \\
\hline Max good field radius & $19.5 \mathrm{~mm}$ & $19.5 \mathrm{~mm}$ \\
\hline Min inner radius & $36.5 \mathrm{~mm}$ & $36.5 \mathrm{~mm}$ \\
\hline Max outer radius & $70.2 \mathrm{~mm}$ & $69.3 \mathrm{~mm}$ \\
\hline Max field in good field region & $0.56 \mathrm{~T}$ & $0.64 \mathrm{~T}$ \\
\hline Max field at “pole tip" & $1.05 \mathrm{~T}$ & $0.97 \mathrm{~T}$ \\
\hline
\end{tabular}


These parameters were derived from optimization of the cell in the Muon1 tracking code, subject to the various constraints described above, plus additional optical stability conditions. The figure of merit for the optimization was to reduce the largest outer magnet radius in the cell, since window-frame correctors are under consideration for being wrapped outside these magnets, meaning a small radius will reduce power requirements as well as being more economical on magnet material

\subsubsection{Comparison of Features vs. Iron Poled Magnets}

A summary table is provided below to illustrate the technology differences between choosing a Halbach magnet design and an iron-dominated permanent magnet design in an accelerator. Permanent magnet materials all have temperature dependence and this can be compensated in the magnet in various ways. The iron quadrupole uses a technique from the Fermilab recycler where the permanent magnet blocks sandwiched in the iron yoke are mixed with NiFe alloy whose magnetization contribution varies in the opposite way as magnetization of the blocks, to provide a temperature range of $20^{\circ} \mathrm{C}$ or more with virtually no field strength variation. In the Halbach magnets, the field and magnetization directions are not parallel, so this method does not work because the NiFe alloy would not provide compensating magnetization in the correct direction. Instead, the linear correctors, which would be present in the design anyway, are used to compensate the field variation, which manifests as an overall reduction in field strength and is therefore also linear (dipole + quad) for these magnets (as shown in Table 2 bellow).

Table 1.9.1.1: Comparison between Hybrid-iron magnets and Halbach magnets

\begin{tabular}{|c|c|c|}
\hline $\begin{array}{c}\text { Field quality + } \\
\text { tuning }\end{array}$ & $\begin{array}{c}\text { Iron Poles } \\
\text { Adjustment would be via conventional } \\
\text { pole shimming. }\end{array}$ & $\begin{array}{c}\text { Hetermined by block magnetization } \\
\text { vectors. Adjustment via floating } \\
\text { shims/iron wires just inside aperture. }\end{array}$ \\
\hline $\begin{array}{c}\text { Field strength + } \\
\text { tuning }\end{array}$ & $\begin{array}{c}\text { Iron shunts to partially short-circuit flux } \\
\text { applied to outside. Also block pre- } \\
\text { measurement and sorting. EM quad } \\
\text { corrector coils around poles. }\end{array}$ & $\begin{array}{c}\text { Determined by block magnetization } \\
\text { vectors. Tune with EM normal quad } \\
\text { and dipole online correctors (see } \\
\text { 'correctors' below). }\end{array}$ \\
\hline $\begin{array}{c}\text { Temperature } \\
\text { sensitivity + } \\
\text { compensation }\end{array}$ & $\begin{array}{c}\text { 0.1\%/K for NdFeB but can (at } ~ \\
\text { strength penalty) incorporate NiFe } \\
\text { material to passively compensate. }\end{array}$ & $\begin{array}{c}\text { 0.1\%/K for NdFeB, cancelled by using } \\
\text { EM normal quad and dipole online } \\
\text { correctors or water cooling }\end{array}$ \\
\hline $\begin{array}{c}\text { Cross-talk in } \\
\text { doublet + } \\
\text { compensation }\end{array}$ & $\begin{array}{c}\text { Few percent cross talk, can be corrected } \\
\text { with shunts. }\end{array}$ & $\begin{array}{c}\text { Negligible cross talk, mu 1 linear } \\
\text { field superposition. }\end{array}$ \\
\hline $\begin{array}{c}\text { Correctors } \\
\text { (online/EM) }\end{array}$ & $\begin{array}{c}\text { Normal quadrupole can be coils would } \\
\text { around each pole. Others require } \\
\text { special coils put within the bore (still } \\
\text { being designed). }\end{array}$ & $\begin{array}{c}\text { Window-frame outside Halbach } \\
\text { magnet using field superposition, } \\
\text { because Halbach is magnetically } \\
\text { transparent. }\end{array}$ \\
\hline
\end{tabular}

To compensate temperature changes, the correctors could be set either using data from the orbit position feedback, or a local field monitor. If the corrector coils themselves are water-cooled (as they are in the most recent design), it is possible to circulate a layer of water just outside the Halbach magnet 
blocks first, to stabilize their temperature to the extent that temperature compensation of any sort is no longer needed.

\subsubsection{Halbach Magnet Design}

The optimized FFAG cell required the QF magnet to be very close to a symmetrical quadrupole, i.e. with zero fields at the center. To simplify matters, the bore location was adjusted slightly so that QF really was exactly symmetrical, so that its design is that of a conventional Halbach quadrupole. Cross-sections of the two magnets are shown in the figures below.

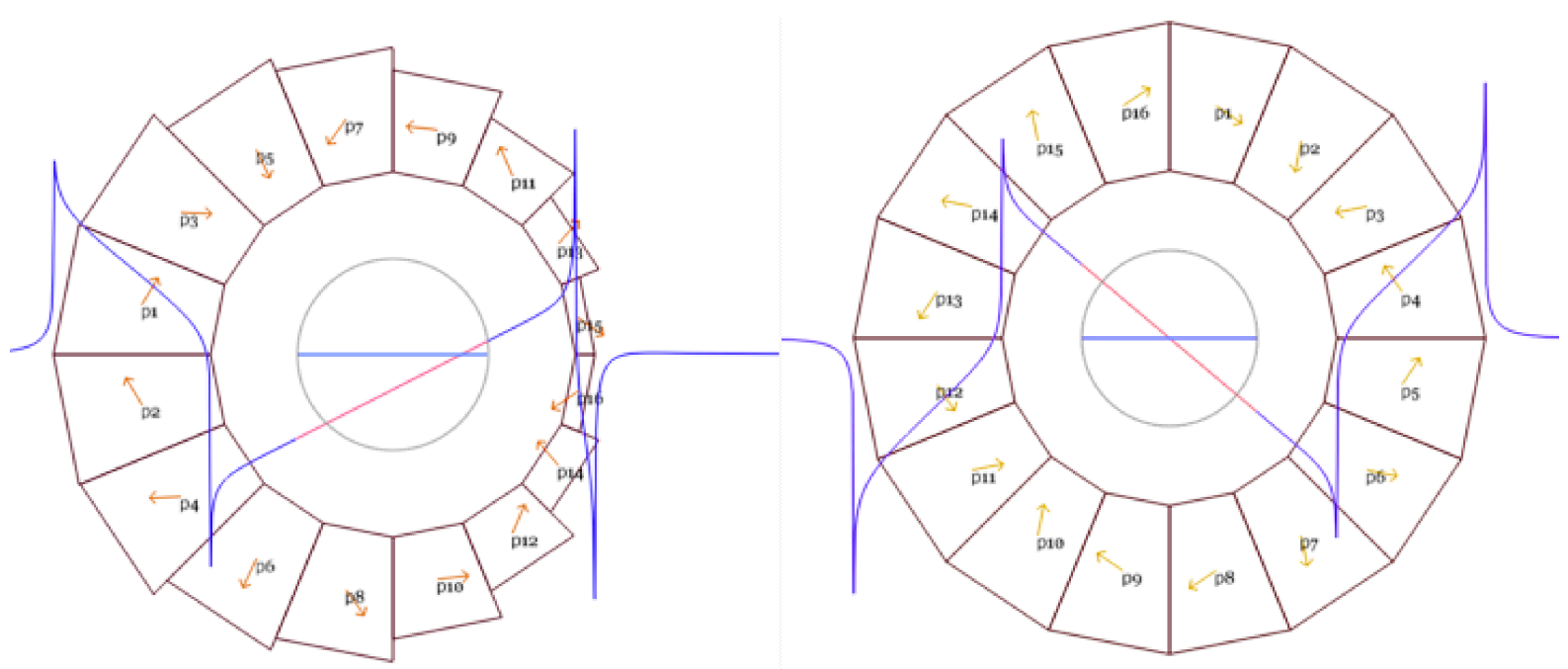

Figure 19: (Left) BD magnet. (Right) QF magnet. Orange graph is vertical field component B_y on the $y=0$ axis, with varying $x$ position. The green segment is the field within the good field region, which is supposed to be linear. Orange arrows in each block show magnetization axis.

The BD magnet on the other hand contains a significant dipole component. In fact, all the beams go through the negative B_y field region, which bends negative electrons clockwise in the LOE hall. There are two reasons some positive $B_{-} y$ is included in the good field region: a request from the vacuum chamber designers that the inner bores of the two magnets be the same, which meant some slack space in $\mathrm{BD}$ where the beams are closer together. Moving BD towards the low-field region most efficiently achieved this. The other reason is that including the $B \_y=0$ point in the good field region allows this magnet to be shifted sideways during the smooth transition from FFAG arc to FFAG straight. The FFAG straight contains magnets with the same gradients but zero dipole component, and the beams travelling along the $B_{-} y=0$ center line. So shifting this $B D$ design provides intermediate magnets in the matching transition, without the beams going outside the good field regions.

The design of the BD magnet is also not a conventional Halbach arrangement: it requires a combination of dipole and quad, whereas conventional annular arrangements can only do one pure multipole at a time. It was considered to nest conventional dipole and quadrupole Halbach magnets but the outer magnet has to be quite large in that case. Stephen Brooks noticed that on one side of the nested magnet, the magnetizations were mostly cancelling anyway, so tried optimizing a design with only a single layer of permanent magnet wedges, but with variable thickness and different magnetization directions. This achieved a very accurate $\left(<10^{\wedge}-5\right.$ in the linear model) combined function integrated 
field as required, a result that was replicated to high accuracy $\left(\sim 10^{\wedge}-4\right)$ by OPERA-3D simulations. It also uses much less material than a nested design

\subsubsection{Magnet Simulation and Codes}

Two codes were used in the design and simulation of these Halbach magnets. The simpler of the two is PM2D written by Stephen Brooks, which is a current sheet approximation of the fields from permanent magnet polygons in 2D. This provides quite a good model of the "average" field (integrated field divided by permanent magnet piece length) through the magnet, provided two conditions hold:

- The materials stay in the linear part of their B-H curve. In fact, if this is violated, the magnets will experience permanent demagnetization, so any valid design ought to satisfy this condition. PM2D can also evaluate the demagnetizing flux from the other blocks at any point to ensure it does not go beyond the coercive force $\left(\mathrm{H}_{-} \mathrm{cj}\right)$ of the material.

- $\quad \mu_{-} r=1$ for all materials. This is almost true of NdFeB, which has a $\mu \_r$ of about 1.025 .

PM2D was used for the initial optimization of the wedge sizes in the BD magnet, which tried to reduce the error multipoles to zero by changing their thickness and magnetization direction independently keeping the required symmetry in the $\mathrm{y}=0$ mid-plane. This requires many iterations of the design to be simulated, so a faster code is preferred during this design stage, before coordinates of the wedge corners are generated as input for the 3D magnet simulation.

The second code used, by Nick Tsoupas for 3D simulations, is OPERA-3D, which is industry standard. Very good agreement was attained between the two codes (on integrated field multipoles) when the materials were not in the demagnetizing regime. Once the design was set, OPERA-3D was always used to do the final simulation and 3D field map generation.

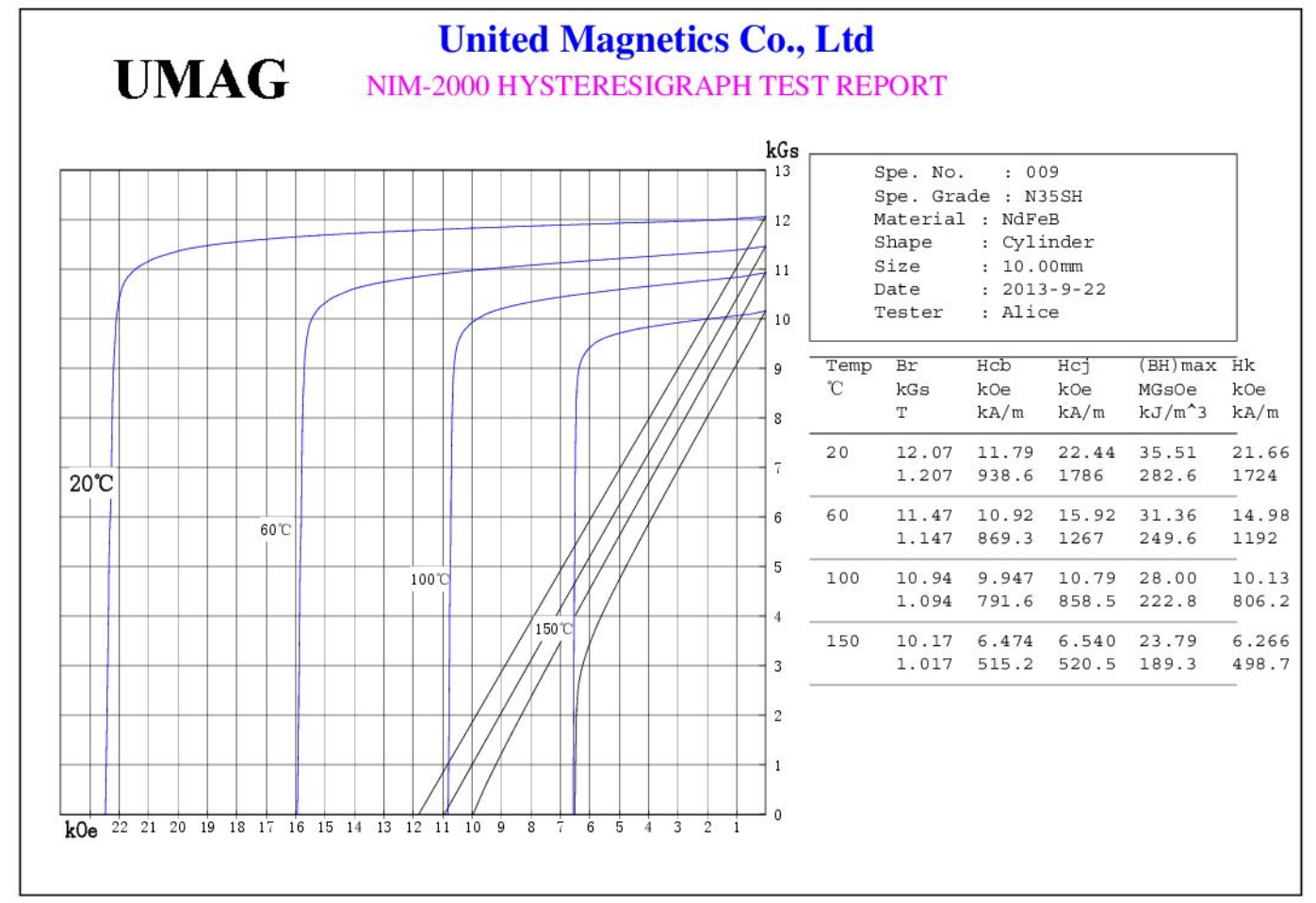

Figure 20: B-H curve of the AllStar Magnetics N35SH NdFeB permanent material, at various temperatures. 
Running in OPERA-3D required that a specific material grade and $\mathrm{B}-\mathrm{H}$ curve be chosen for the permanent magnet blocks. These grades and curves vary by manufacturer, so a grade from AllStar Magnetics was selected, which is the manufacturer for blocks for the CBETA prototype magnets currently under order and shipping at the end of March 2016. The grade N35SH was selected, which combines a medium strength of 35 MGauss.Oe (the maximum available being $~ 52$ MGauss.Oe) with a good resistance to external demagnetizing fields. This is what the " $\mathrm{SH}$ " suffix means: a strong resistance to heat, which stems from its high $\mathrm{H}_{\text {c }}$ j demagnetizing field value $(2.24 \mathrm{~T})$ at room temperature. The strength translates into a residual field B_r of 1.207 T.

After OPERA-3D models were run, a best fit with the magnetization "B_r" value used in PM2D, which assumes $\mu_{-} r=1$, was found (1.1939 T), which represents the average magnetization from the material including the small reduction from regions of reverse flux with $\mu_{-} r$ being slightly larger than 1 . This lies between $\mathrm{B} \_r$ and $\mathrm{H}_{-} \mathrm{cb}$ of the material as expected. With this value, the PM2D designs could be loaded directly into OPERA-3D (with the N35SH material) and the strength would be correct, with no further design modifications required.

\subsubsection{Tracking and Compatibility with FFAG Lattice}

Once OPERA-3D field maps have been generated, they can be loaded back in to the Muon 1 tracking code, which is the same code used for the original lattice optimization done with field models rather than field maps.

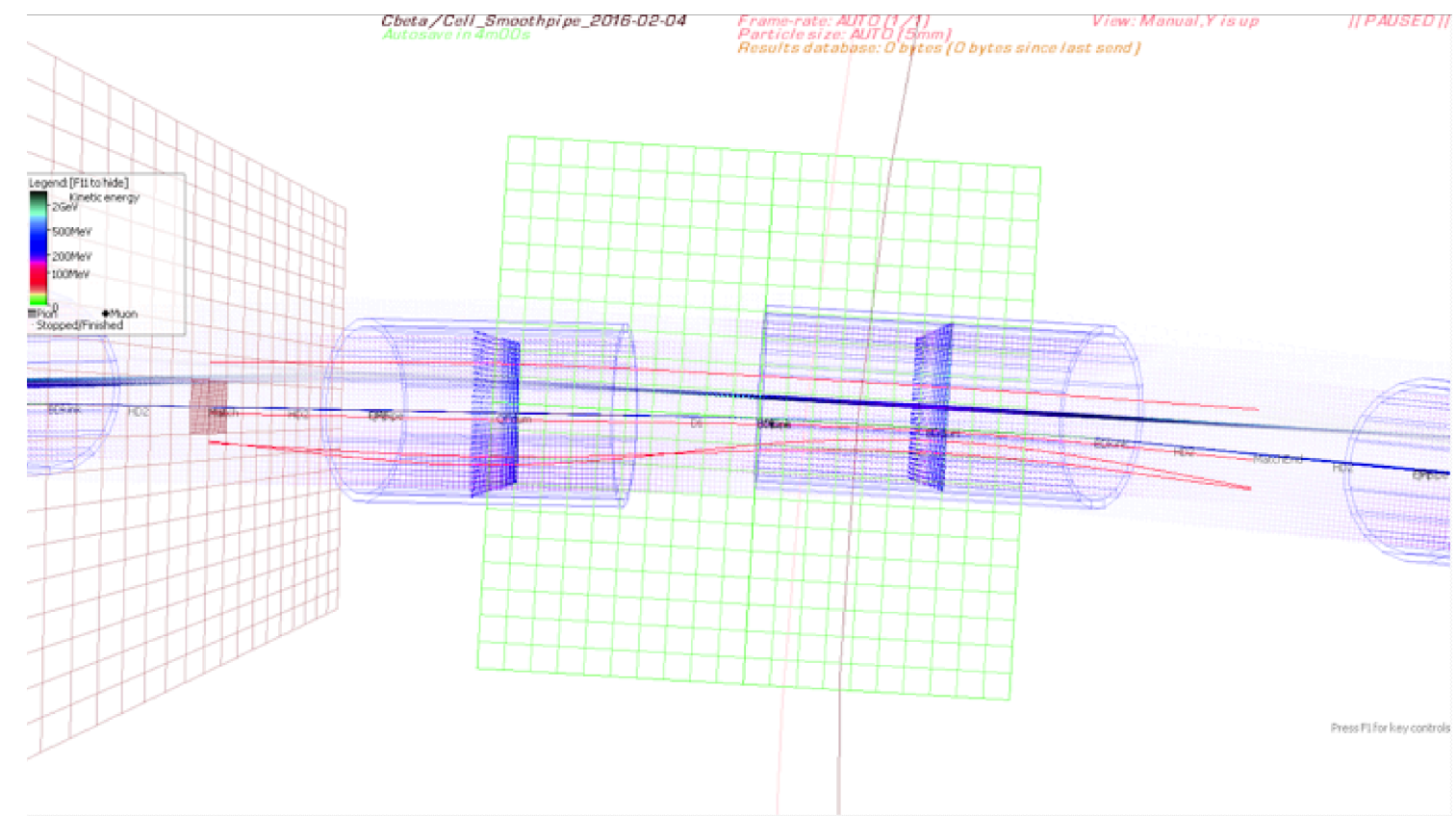

Figure 21: Matched orbits (green) for the four CBETA energies through an FFAG arc cell made with OPERA-3D field maps generated from Halbach magnets. The orange cylinders represent the approximate apertures of the vacuum pipe and the grids are $1 \mathrm{~cm}$ per square.

A simulation by Stephen Brooks with a program Muon1, shown in Fig. 21, finds the "closed orbits" for each energy, which exit the cell at the same position and angle that they enter. The closed orbits found through field maps will be slightly different than those found for the original field model in the latticedesign optimization, but as shown in the graph and table below, the discrepancy is not very large $(<1 \mathrm{~mm})$. This good agreement is partly due to a fortunate choice of fringe field length in Muon1's soft- 
edged Maxwellian field model. Muon1 models the fall-off of multipole components near the entrance of exit of a magnet as proportional to: $1 / 2+1 / 2 \tanh (z / f)$ where $z$ is the longitudinal position relative to the magnet end and $f$ is a "fringe length" parameter ( $f$ ). It was chosen to be $2.5 \mathrm{~cm}$ here, roughly the same order of size as the magnet apertures. Detailed studies suggested the best agreement with these field maps is obtained with $f=2.7 \mathrm{~cm}$. For these short magnets in CBETA, the fringe field makes up a large part of the field so it is important to include it consistently (some hard-edged models do not have good agreement with the optic

Table 19.4.1

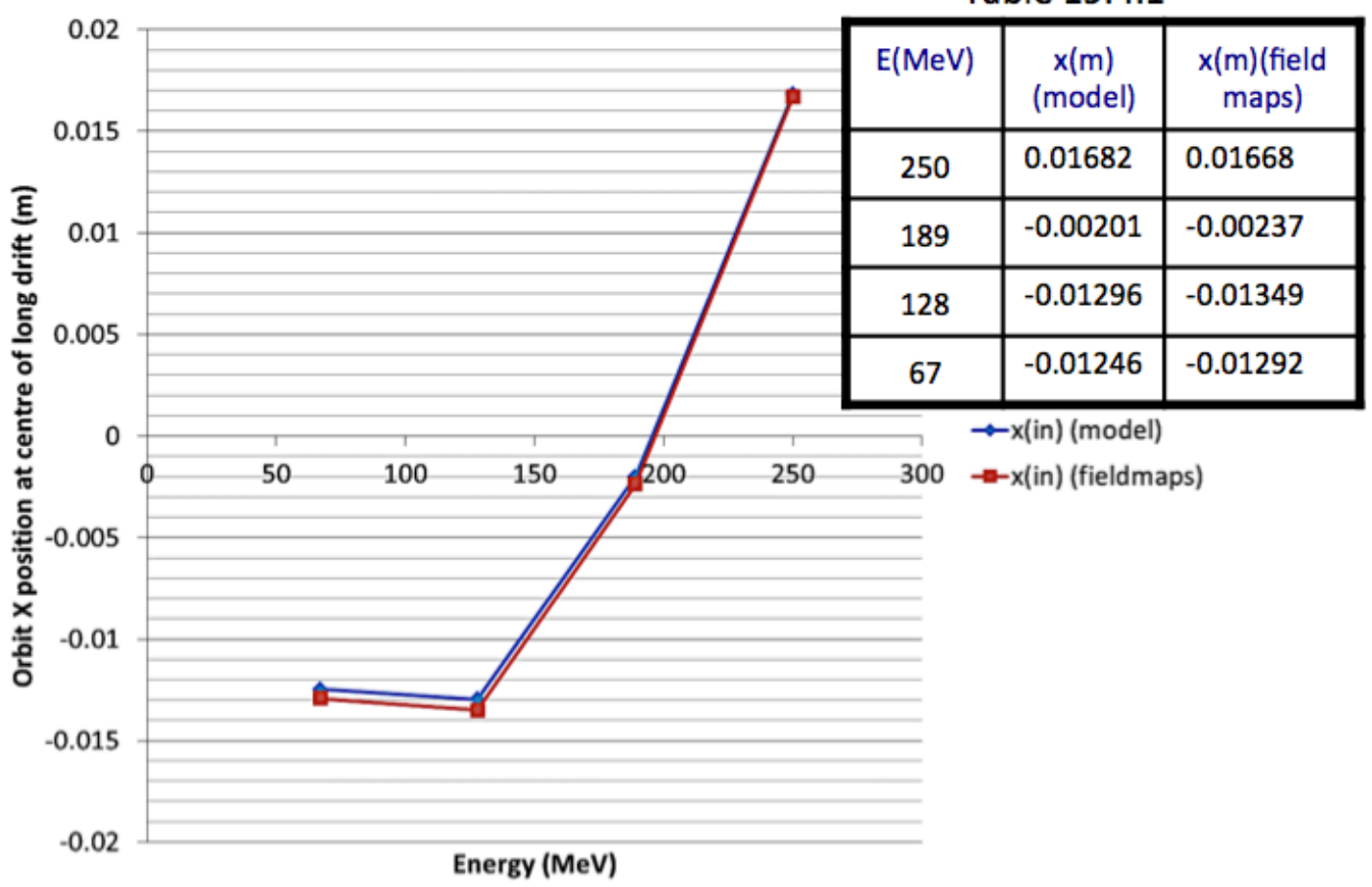

Figure 22: \& Table 1.9.4.1 Transverse position ( $X$, in meters) of the four closed orbits, as a function of energy, at the midpoint of the long drift in the CBETA FFAG arc cell. Blue dots are from a Muon1 simulation using field models and red dots from a Muon1 simulation using OPERA-3D field maps.

The closed orbit matching process also determines the shape of the beam (optical alpha and beta functions) that will be preserved on traversing once through the cell. This also allows the single-cell tunes in the $X$ and $Y$ planes to be calculated. A similar comparison of tunes from the field map versus the original optimizer's field model is shown in the figure and table below. 


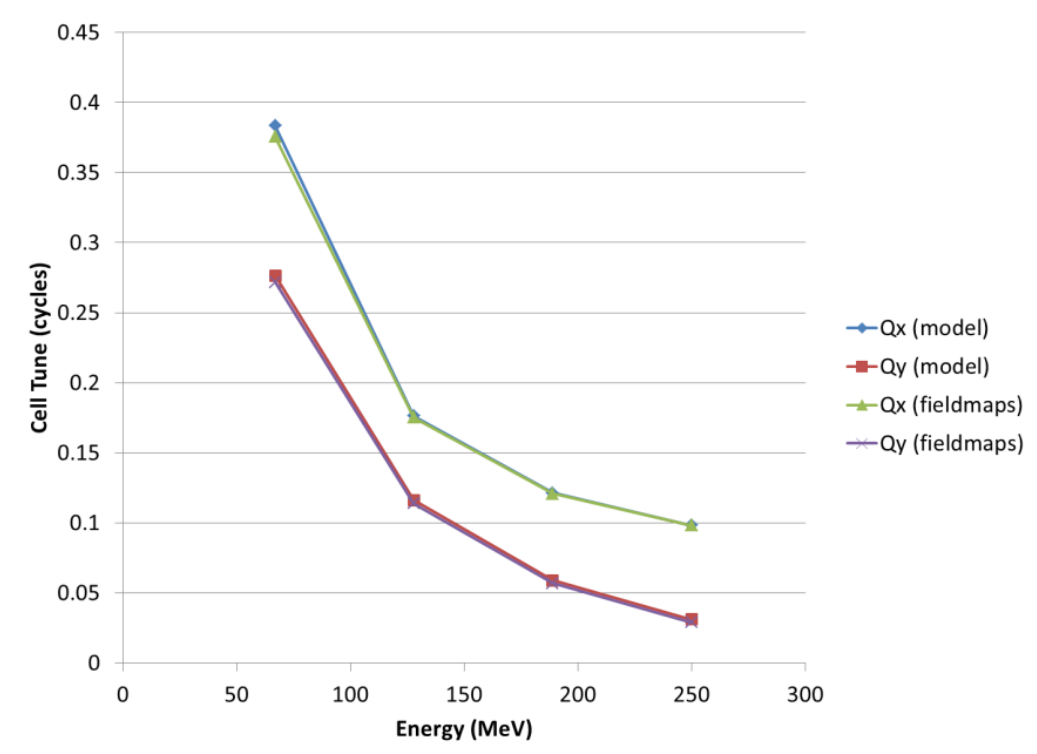

Figure 23: Comparison of the calculated $X$ and $Y$ tunes of the FFAG cell using Muon1's model field and OPERA-3D field maps.

Table 19.4.2:

\begin{tabular}{|l|l|l|l|l|}
\hline $\begin{array}{l}\text { Energy } \\
\text { (MeV) }\end{array}$ & $\mathrm{Q}_{\mathrm{x}}$ (model) & $\mathrm{Q}_{\mathrm{y}}$ (model) & $\begin{array}{l}\mathrm{Q}_{\mathrm{x}} \text { (field } \\
\text { maps) }\end{array}$ & $\mathrm{Q}_{\mathrm{y}}$ (field maps) \\
\hline $\mathbf{2 5 0}$ & 0.098132 & 0.031006 & 0.098272 & 0.029062 \\
\hline $\mathbf{1 8 9}$ & 0.121474 & 0.058797 & 0.120911 & 0.056874 \\
\hline $\mathbf{1 2 8}$ & 0.176315 & 0.11615 & 0.175274 & 0.113949 \\
\hline $\mathbf{6 7}$ & 0.383309 & 0.276579 & 0.375643 & 0.271753 \\
\hline
\end{tabular}

The cell tunes are important because they determine the limits on the stability of the beam ( 0 and 0.5 being the unstable limits) and its response to errors, the tune determining the frequency of error oscillations. The largest discrepancy between field map and model field is found in the low-energy $(67 \mathrm{MeV})$ beam, where the model predicts 0.3833 and the field maps predict 0.3756 , a difference of 0.0077 cycles per cell. This is not a large enough difference to put the beam into a resonance or drastically affect the optical behavior of the machine.

\subsubsection{Window-Frame Correctors}

The property of the permanent magnets to be magnetically saturated allows superposition of the magnetic fields therefore permanent magnets can accept electromagnets as corrector magnets with no distortion of the their magnetic field. Figure1 is an isometric view of an OPERA model of a Halbach-type magnet surrounded by a window frame electromagnet acting as a corrector. 


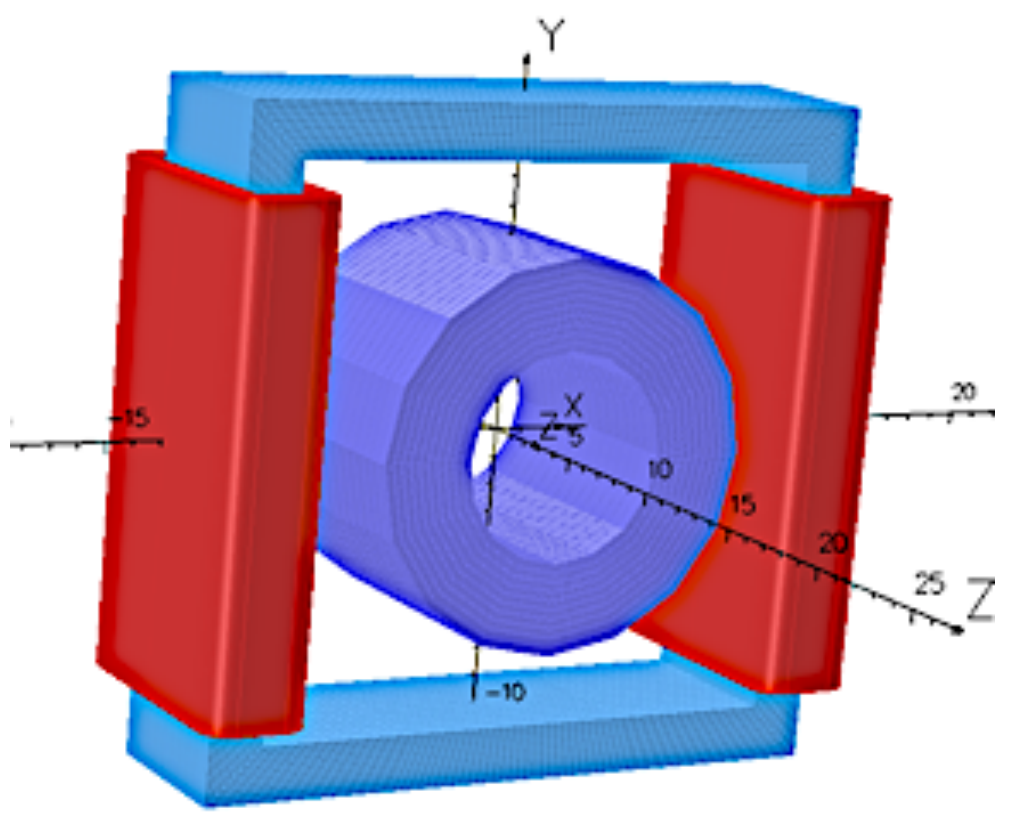

Figure 24: Window frame magnet with two coils generates a normal dipole field, which is superimposed on the field of the permanent magnet.

In this section we will present results from the 3D OPERA calculations, which prove the following statements:

1. The window frame magnets in spite their large aperture and short length, do not excite significant transverse magnetic multipoles except the ones are designed to produce.

2. An excited window frame magnet placed around a Halbach-type permanent magnet as in Fig. 1 does not alter significantly the multipoles of the Halbach-type magnet (measurements are under way) and there is an almost perfect superposition of the fields of the two magnets.

3. The Halbach-type magnets lend themselves easily to window-frame corrector magnets and do not interfere with possible access to the beam instrumentation which is placed in the short drift spaces between the magnets.

4. Many Halbach-type magnets placed in conduct along their symmetry axis provide an almost perfect field superposition. (Measurements have been made thus no results from calculations will bee sent).

\subsubsection{The B field of a Window-Frame Electromagnet}

Figure 25 is a picture of 3D OPERA model window frame magnet to be used as corrector around a Halbach-magnet. 


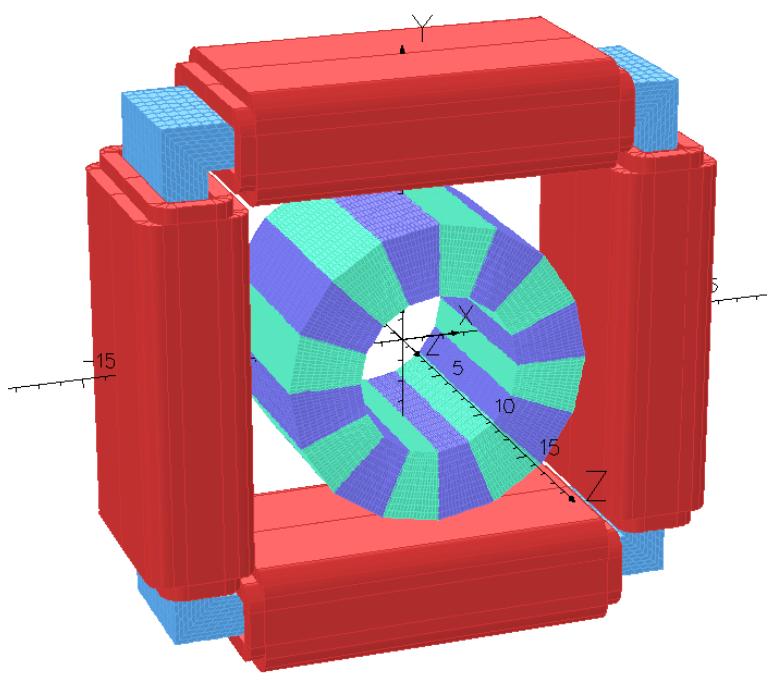

Figure 25: A window frame magnet with eight coils acting as normal and skew dipoles, and a normal quadrupole. By rotating the window frame by $45^{\circ}$ we can generate a skew quadrupole instead of normal one.

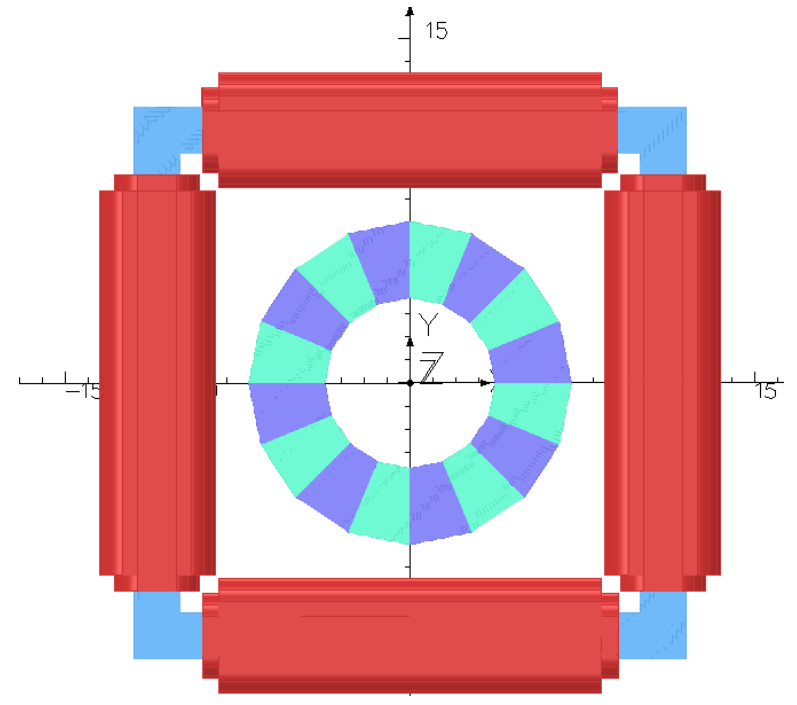

Figure 26: The projection of the window frame magnet on the $x, y$ plane. The maximum transverse extend of the magnet is less than $30 \mathrm{~cm}$.

Figure 27 is a projection on the $x$, $y$ plane of the window frame magnet which shows that the maximum transverse directions of the corrector magnet surrounding a Halbach magnet is less than $30 \mathrm{~cm}$ Table I shows the integrated magnetic multipoles at $R=1 \mathrm{~cm}$ of three different configurations of a quadrupole Halbach magnet and dipole window frame magnet. The $2^{\text {nd }}$ row shows the integrated multipoles a dipole window frame magnet with no permanent magnet inside. Row 3 shows the integrated multipoles of a quadrupole Halbach-type magnet with no excitation of the dipole corrector and row 4 the multipoles of the dipoles window frame magnet excited, surrounding the quadrupole Halbach-type magnet. The permanent magnet material of the quadrupole magnet is NdFeB-N35 and the BH-curve for this material is shown in Fig. 4. The results from Table I show that the field of the window frame magnet is simply superimposed on the field of the quadrupole magnet. 


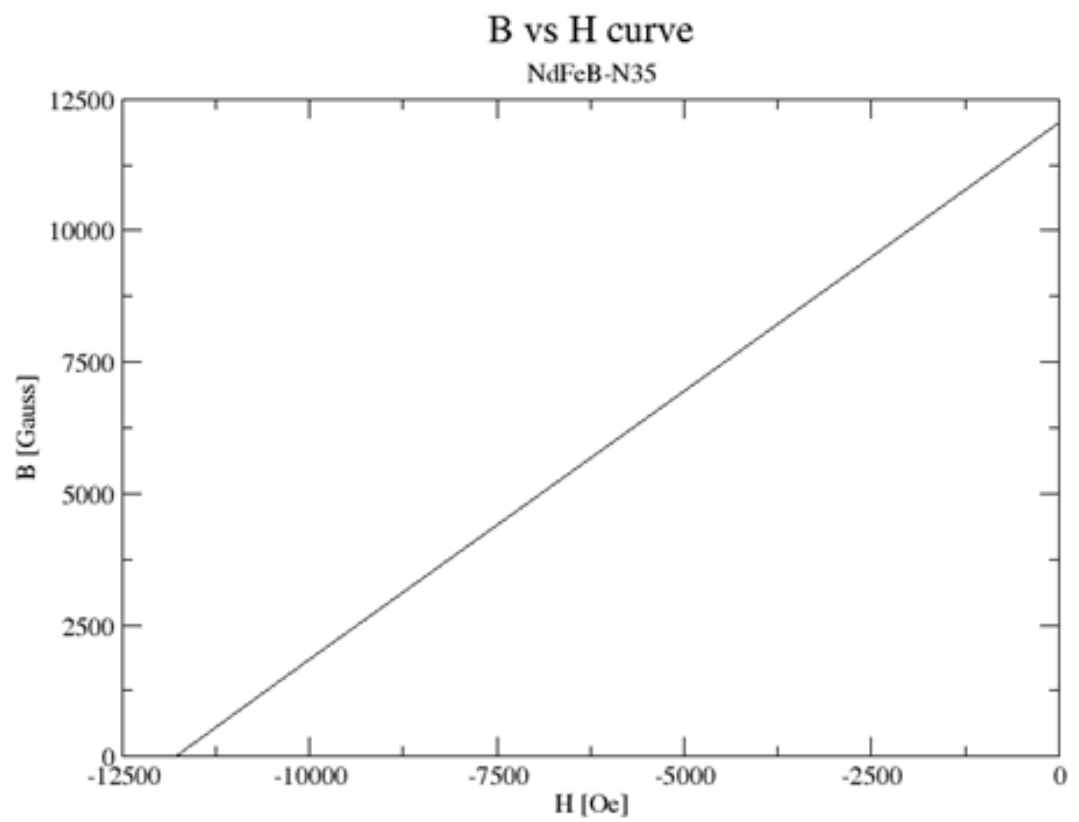

Figure 27. The B-H curve of the NdFeB-N35 material.

Table 19.6.1: The integrated magnetic multipoles of the window frame magnet by itself ( $2^{\text {nd }}$ row) of a quadrupole Halbach type magnet $\left(3^{\text {rd }}\right.$ row), and of the window frame magnet surrounding the quadrupole Halbach-type magnet.

\begin{tabular}{|l|l|l|l|l|l|l|}
\hline & $\begin{array}{l}\text { Dipole } \\
{[\text { Gauss.cm }]}\end{array}$ & $\begin{array}{l}\text { Quad } \\
{[\text { Gauss }]}\end{array}$ & $\begin{array}{l}\text { Sext. } \\
{[\text { Gauss.cm }}\end{array}$ & $\begin{array}{l}\text { Oct. } \\
{[\text { Gauss.cm }}\end{array}$ & $\left.\begin{array}{l}\text { Dec. } \\
{[\text { Gauss.cm }}\end{array}\right]$ & $\left.\begin{array}{l}\text { 12pole } \\
{[\text { Gauss.cm }}\end{array}\right]$ \\
\hline WF only & 1931.14 & -0.0013 & 1.02 & 0.00003 & 0.014 & -0.000015 \\
\hline PM only & 0.000003 & 27798.5 & 0.000003 & 0.00000002 & 0.000003 & 0.037 \\
\hline WF_PM & 1933.7 & 27798.5 & 1.02 & 0.0123 & 0.017 & 0.016 \\
\hline
\end{tabular}

Figure 28 is an isometric view few of permanent magnets of the $c \beta$ arc with correctors. This view shows that the window frame magnets do not extend into the drift space between the magnets. The projection on the $y, z$ plane of the six magnets is shown in in Fig. 29. The current through the coils of the window frame magnet can generate the required correction field for the permanent magnets.

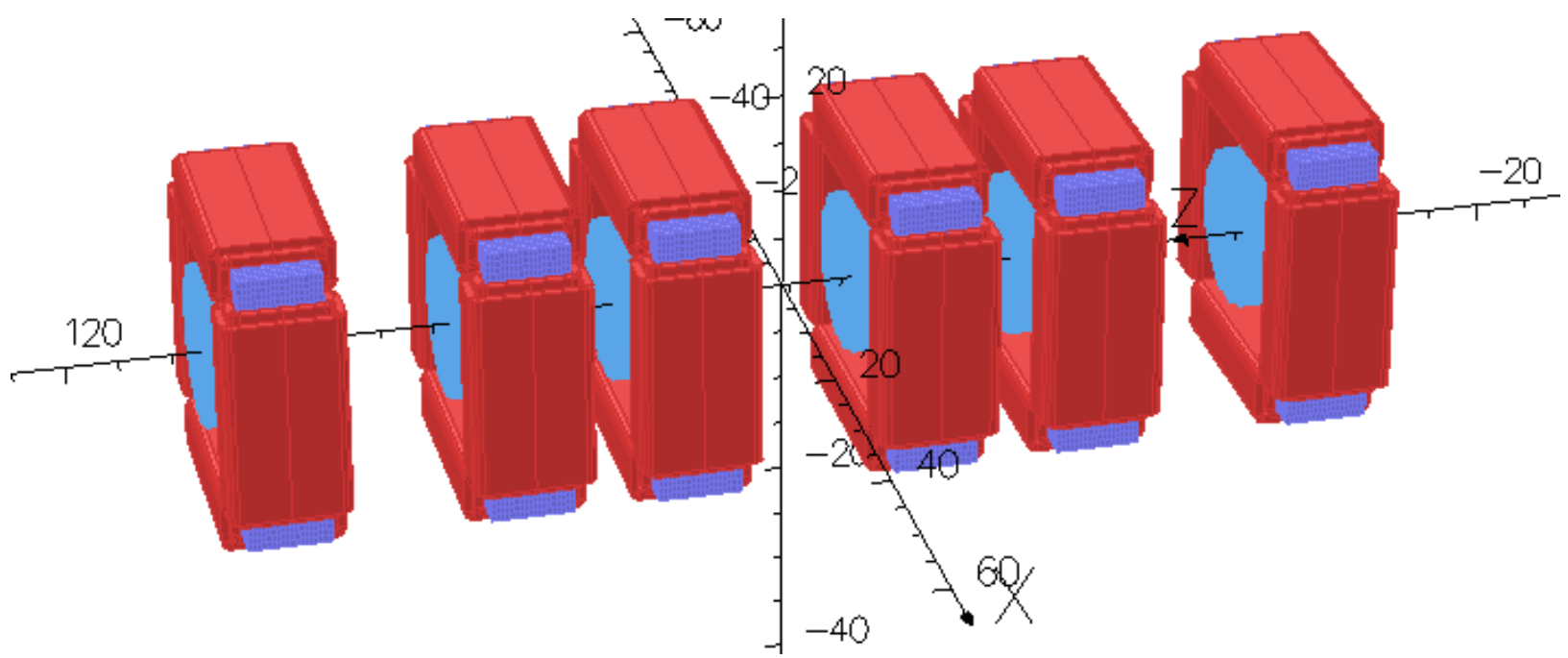

Figure 28: Isometric view of six of the permanent magnets of the CBETA. 


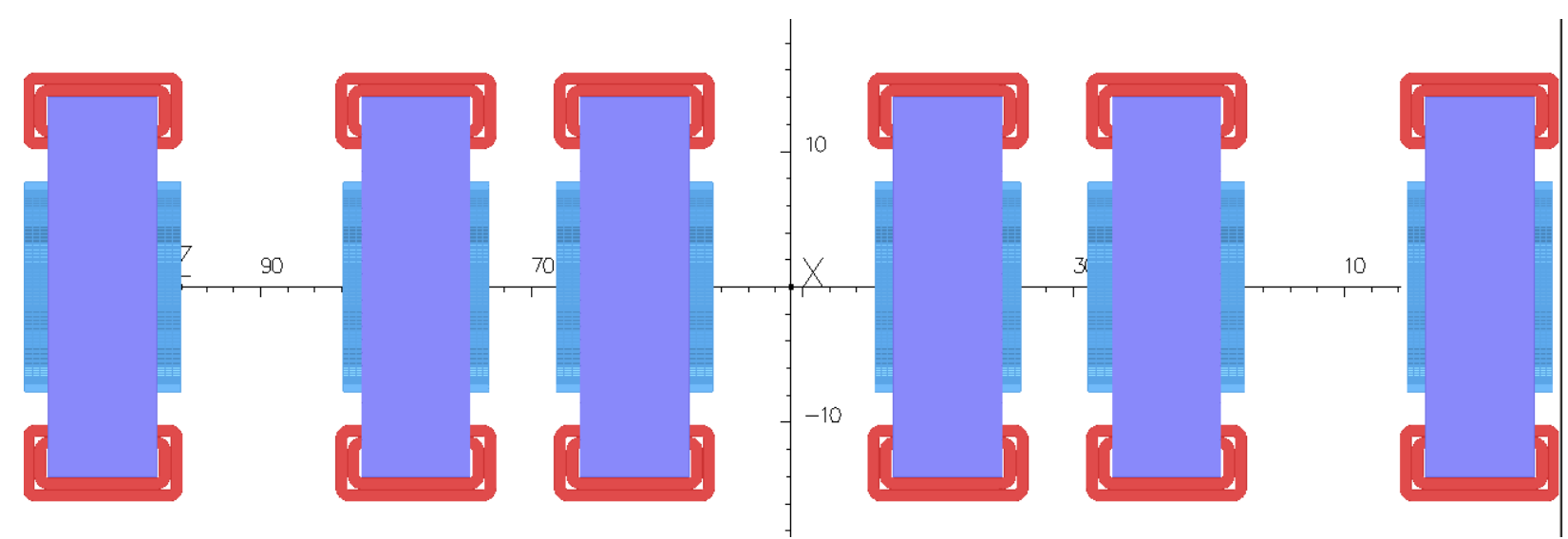

Figure 29. Projection on the $y, z$ plane of the six permanent magnets of the CBETA arc.

\subsubsection{Halbach Magnet R\&D and Shimming Results}

BNL lab-directed R\&D (LDRD) provided some money for constructing prototype permanent magnet quadrupoles for eRHIC, which is also an FFAG. Blocks were ordered from Shin-Etsu Corporation in August 2014 for three different designs, one of which was a Halbach quadrupole. The main differences between eRHIC and CBETA magnets are that eRHIC requires an open mid-plane to allow synchrotron radiation to be dumped and eRHIC's magnets are $\sim 1 \mathrm{~m}$ long, an order of magnitude longer than CBETA's. However, to reduce cost, the eRHIC prototype magnets were built in $6 \mathrm{~cm}$ sections, roughly the longest piece of permanent magnet the company could magnetize at once.

The table below shows that the eRHIC prototype Halbach quadrupole is a good model for the CBETA magnets too; at least until the parts for purpose-built CBETA prototypes are delivered.

Table 1.9.7.1: Comparison of the Halbach shimming test magnet " $5 A$ " with requirements of CBETA magnets.

\begin{tabular}{|l|l|l|l|}
\hline Parameter & $\begin{array}{l}\text { eRHIC prototype } \\
\text { quad “5A" }\end{array}$ & $\begin{array}{l}\text { C } \beta \text { requirement } \\
\text { QF }\end{array}$ & $\begin{array}{l}\text { C } \beta \text { requirement } \\
\text { BD }\end{array}$ \\
\hline Length & $60.0 \mathrm{~mm}$ & $96.3 \mathrm{~mm}$ & $126.4 \mathrm{~mm}$ \\
\hline Gradient & $27.5 \mathrm{~T} / \mathrm{m}$ (measured) & $-28.8 \mathrm{~T} / \mathrm{m}$ & $19.2 \mathrm{~T} / \mathrm{m}$ \\
\hline Central dipole & 0 (by realignment) & 0 & $-0.268 \mathrm{~T}$ \\
\hline Material & $\begin{array}{l}\text { SmCo R26HS } \\
\text { (Shin-Etsu) }\end{array}$ & $\begin{array}{l}\text { NdFeB N35SH (AllStar } \\
\text { Magnetics) }\end{array}$ & $\begin{array}{l}\text { NdFeB N35SH (AllStar } \\
\text { Magnetics) }\end{array}$ \\
\hline $\begin{array}{l}\text { Min R of physical } \\
\text { magnet pieces }\end{array}$ & $\begin{array}{l}22.5 \mathrm{~mm} \text { (design) } \\
23.5 \mathrm{~mm} \text { (measured) }\end{array}$ & $36.5 \mathrm{~mm}$ & $36.5 \mathrm{~mm}$ \\
\hline Max R of beam centroid & $\begin{array}{l}10 \mathrm{~mm} \text { (rotating coil) } \\
15 \mathrm{~mm} \text { (extrapolated) }\end{array}$ & $19.5 \mathrm{~mm}$ & $19.5 \mathrm{~mm}$ \\
\hline $\mathbf{R}_{\text {max,beam } / \mathbf{R}_{\text {min,magnet }}}$ & $\begin{array}{l}43 \% \text { (coil) } \\
64 \% \text { (extrapolated) }\end{array}$ & $53 \%$ & $53 \%$ \\
\hline
\end{tabular}


The eRHIC magnet was constructed out of SmCo instead of NdFeB for historical reasons: concerns about radiation resistance, with SmCo being more resistant. Since then, a radiation test has shown $\mathrm{NdFeB}$ of an appropriate grade survives $>100 \mathrm{~Gy}$ of radiation on the RHIC beam dump during a run. SmCo also contains much more cobalt, which can lead to long-term Co-60 activation.

\subsubsection{Repeatability of Un-shimmed Halbach Magnets}

Five $6 \mathrm{~cm}$-long permanent magnet quadrupoles were made for eRHIC prototyping, of the kind shown in the figure below. Note that the holder was made on a 3D printer and the design for eRHIC incorporates mid-plane gaps for synchrotron radiation to exit. These were all measured on the BNL magnet division rotating coil to test the field quality of Halbach quadrupoles without shimming.

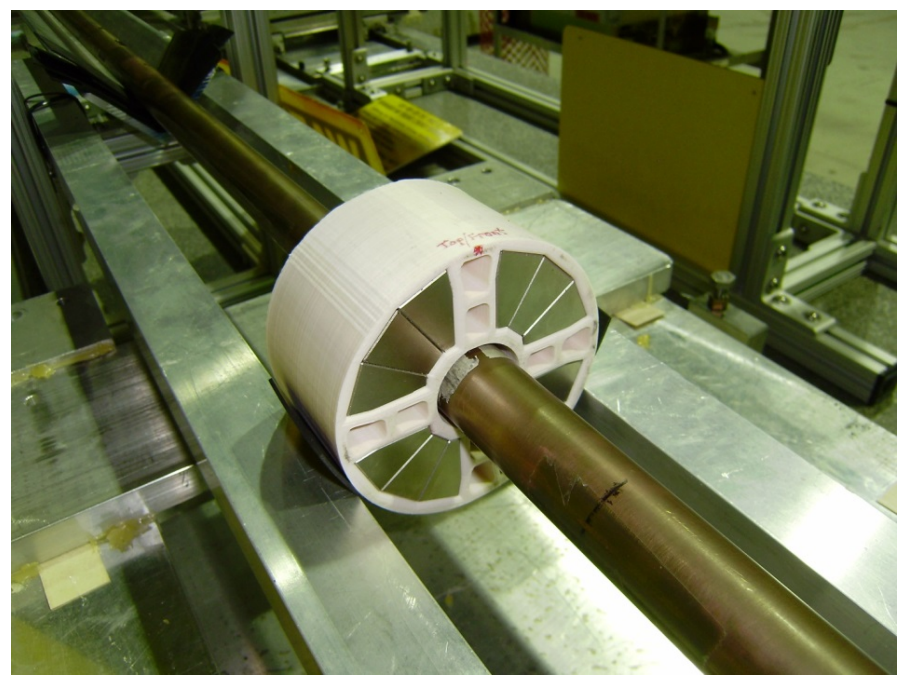

Figure 30. The $6 \mathrm{~cm}$-long eRHIC LDRD Halbach magnet measured on the rotating coil at BNL's magnet division.

Table 1.9.81: Rotating coil measurements of 5 un-shimmed Halbach permanent magnet quadrupoles.

Summary of field measurements in eRHIC Permanent Magnet Quadrupoles (27-Apr-2015)

Field harmonics are in "units" of $10^{-4}$ of the quadrupole field at a reference radius of $10 \mathrm{~mm}$.

\begin{tabular}{|l|c|c|c|c|c|}
\hline Quantity & $\begin{array}{c}\text { PMQ_0001 } \\
\text { Run 2 }\end{array}$ & $\begin{array}{c}\text { PMQ_0002 } \\
\text { Run 3 }\end{array}$ & $\begin{array}{c}\text { PMQ_0003 } \\
\text { Run 2 }\end{array}$ & $\begin{array}{c}\text { PMQ_0004 } \\
\text { Run 3 }\end{array}$ & $\begin{array}{c}\text { PMQ_0005 } \\
\text { Run 2 }\end{array}$ \\
\hline Integrated Gradient (T) & 1.8647 & 1.9097 & 1.9053 & 1.8958 & 1.9024 \\
\hline Normal Dipole & -- & -- & -- & -- & -- \\
\hline Normal Quadrupole & 10000.00 & 10000.00 & 10000.00 & 10000.00 & 10000.00 \\
\hline Normal Sextupole & 27.83 & -29.83 & 35.83 & -4.53 & -11.95 \\
\hline Normal Octupole & 5.39 & -3.12 & 32.81 & 16.50 & 3.61 \\
\hline Normal Decapole & -4.92 & -2.44 & 2.90 & 7.09 & 3.86 \\
\hline Normal Dodecapole & -188.14 & -194.57 & -188.00 & -192.96 & -190.26 \\
\hline Normal 14-pole & -1.59 & 0.36 & -0.67 & 0.43 & 1.03 \\
\hline Normal 16-pole & -0.44 & -0.22 & -1.10 & -0.58 & -1.31 \\
\hline Normal 18-pole & -0.24 & 0.19 & -0.38 & -0.25 & 0.07 \\
\hline Normal 20-pole & -2.37 & -2.88 & -3.13 & -2.93 & -2.91 \\
\hline Normal 22-pole & 0.04 & 0.03 & 0.01 & 0.03 & -0.01 \\
\hline Normal 24-pole & 0.02 & 0.00 & -0.02 & 0.01 & 0.04 \\
\hline Normal 26-pole & 0.02 & -0.01 & 0.02 & -0.01 & -0.02 \\
\hline Normal 28-pole & 0.11 & 0.12 & 0.12 & 0.13 & 0.12 \\
\hline Normal 30-pole & 0.00 & 0.00 & 0.00 & 0.00 & 0.00 \\
\hline
\end{tabular}

\begin{tabular}{|l|c|c|c|c|c|}
\hline Quantity & $\begin{array}{c}\text { PMQ_0001 } \\
\text { Run 2 }\end{array}$ & $\begin{array}{c}\text { PMQ_0002 } \\
\text { Run 3 }\end{array}$ & $\begin{array}{c}\text { PMQ_0003 } \\
\text { Run 2 }\end{array}$ & $\begin{array}{c}\text { PMQ_0004 } \\
\text { Run 3 }\end{array}$ & $\begin{array}{c}\text { PMQ_0005 } \\
\text { Run 2 }\end{array}$ \\
\hline Field Angle (mr) & -- & -- & -- & -- & -- \\
\hline Skew Dipole & -- & -- & -- & -- & -- \\
\hline Skew Quadrupole & -- & -- & -- & -- & -- \\
\hline Skew Sextupole & -16.41 & 1.90 & -43.69 & 28.96 & -5.28 \\
\hline Skew Octupole & -12.32 & 0.25 & -12.55 & 4.03 & -18.51 \\
\hline Skew Decapole & -11.98 & -6.08 & -5.68 & -1.00 & -8.52 \\
\hline Skew Dodecapole & -2.27 & -0.99 & -3.12 & 0.87 & -4.96 \\
\hline Skew 14-pole & 1.93 & 0.13 & 0.47 & 0.01 & 0.85 \\
\hline Skew 16-pole & -0.22 & -0.09 & -0.02 & 0.27 & -0.13 \\
\hline Skew 18-pole & 0.03 & 0.17 & -0.06 & 0.05 & 0.10 \\
\hline Skew 20-pole & 0.08 & 0.00 & 0.07 & -0.20 & 0.01 \\
\hline Skew 22-pole & 0.02 & 0.03 & 0.07 & -0.05 & 0.00 \\
\hline Skew 24-pole & 0.01 & 0.00 & 0.02 & -0.02 & 0.01 \\
\hline Skew 26-pole & 0.00 & 0.00 & 0.00 & -0.01 & 0.01 \\
\hline Skew 28-pole & 0.00 & -0.01 & 0.00 & 0.01 & 0.00 \\
\hline Skew 30-pole & 0.00 & 0.00 & 0.00 & 0.00 & 0.00 \\
\hline
\end{tabular}

Blocks from this factory, according to the supplier, typically have 1-2\% magnetization strength error and $\pm 1^{\circ}$ magnetization direction error. The five assemblies were measured on the rotating coil (not all the individual blocks), with results shown in the table below. The Normal Dodecapole error of $\sim-190$ units 
present in all magnets should be ignored for this comparison, since it was a systematic error made by the manufacturer using the wrong information for magnetizing some of the blocks. This was compensated for in later designs by moving the blocks.

The raw magnets have sextupole error magnitudes (normal and skew added in quadrature) of 13-57 units; octupole errors of 3-35 units; decapole errors of 6-13 units, with poles above dodecapole being less than 3 units. A unit is $10^{\wedge}-4$ relative to the main field, so these magnets are slightly better than $1 \%$ relative field error, which is roughly to be expected from the intrinsic magnetization errors of the blocks they are made from. This on its own is not yet good enough for the $10^{\wedge}-3$ level accuracy required by the accelerator, so shimming is required as described in the next section.

The coil is calibrated to measure at $1 \mathrm{~cm}$ radius, which is smaller than the CBETA orbit excursion but as shown in the previous table, the eRHIC prototype magnet had a smaller aperture overall

\subsubsection{Field Quality Improvement after Iron Wire Shimming}

The pieces from eRHIC magnet \#5 were re-used to make a magnet that lacked the dodecapole error and served as a test-bed for shimming, as shown in the figure below.

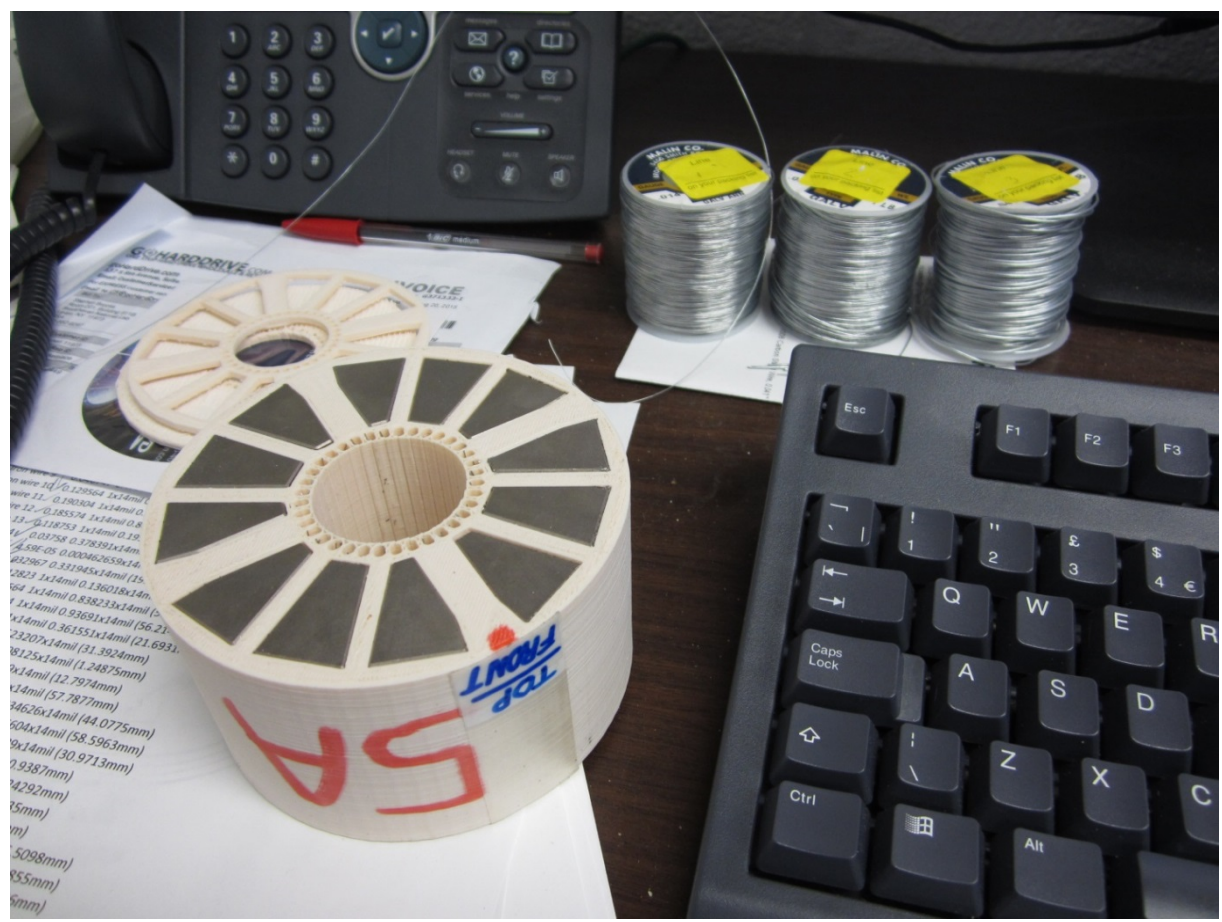

Figure 31: The pieces of the eRHIC LDRD Halbach magnet placed in a new 3D-printed holder to form magnet " $5 \mathrm{~A}$ ". This is a corrected Halbach quadrupole whose holder incorporates holes for iron shims to be placed around the inside of the bore (iron wire grades shown in background).

Table 1.9.9.1: Rotating coil measurements of the shimming test magnet before shimming (Run 1_02), with sextupole-only correction (Run 2) and with all-multipole correction (Run 3) using iron wire shims. 
eRHIC Permanent Magnet Quadrupoles PMQ_0005 \& PMQ_005A (14-Oct-2015)

Field harmonics are in "units" of $10^{-4}$ of the quadrupole field at a reference radius of $10 \mathrm{~mm}$.

\begin{tabular}{|l|c|c|c|c|}
\hline Quantity & $\begin{array}{c}\text { PMQ_0005 } \\
\text { Run 2 }\end{array}$ & $\begin{array}{c}\text { PMQ_005A* } \\
\text { Run 1_02(+) }\end{array}$ & $\begin{array}{c}\text { PMQ_005A* } \\
\text { Run 2(+十) }\end{array}$ & $\begin{array}{c}\text { PMQ_005A* } \\
\text { Run 3(++) }\end{array}$ \\
\hline Integrated Gradient (T) & 1.9024 & 1.6501 & 1.6519 & 1.6537 \\
\hline Normal Dipole & -- & -- & -- & -- \\
\hline Normal Quadrupole & 10000.00 & 10000.00 & 10000.00 & 10000.00 \\
\hline Normal Sextupole & -11.95 & $\mathbf{- 1 9 . 4 6}$ & $\mathbf{- 0 . 5 8}$ & 0.87 \\
\hline Normal Octupole & 3.61 & 5.61 & 5.21 & 3.12 \\
\hline Normal Decapole & 3.86 & -0.99 & -0.84 & -0.32 \\
\hline Normal Dodecapole & $\mathbf{- 1 9 0 . 2 6}$ & $\mathbf{- 1 . 0 3}$ & -1.06 & 0.55 \\
\hline Normal 14-pole & 1.03 & 1.25 & 1.04 & -0.03 \\
\hline Normal 16-pole & -1.31 & -1.47 & -1.52 & -0.24 \\
\hline Normal 18-pole & 0.07 & 0.12 & 0.13 & 0.05 \\
\hline Normal 20-pole & $-\mathbf{2 . 9 1}$ & $\mathbf{0 . 4 4}$ & 0.40 & -0.01 \\
\hline Normal 22-pole & -0.01 & -0.03 & -0.01 & 0.01 \\
\hline Normal 24-pole & 0.04 & 0.05 & 0.03 & -0.09 \\
\hline Normal 26-pole & -0.02 & -0.01 & -0.01 & -0.03 \\
\hline Normal 28-pole & 0.12 & -0.12 & -0.12 & 0.02 \\
\hline Normal 30-pole & 0.00 & 0.00 & 0.00 & 0.00 \\
\hline
\end{tabular}

\begin{tabular}{|l|c|c|c|c|}
\hline Quantity & $\begin{array}{c}\text { PMQ_0005 } \\
\text { Run 2 }\end{array}$ & $\begin{array}{c}\text { PMQ_005A* } \\
\text { Run 1_02(+) }\end{array}$ & $\begin{array}{c}\text { PMQ_005A* } \\
\text { Run 2(+十) }\end{array}$ & $\begin{array}{c}\text { PMQ_005A* } \\
\text { Run 3(++) }\end{array}$ \\
\hline Field Angle (mr) & -- & -- & -- & -- \\
\hline Skew Dipole & -- & -- & -- & -- \\
\hline Skew Quadrupole & -- & -- & -- & -- \\
\hline Skew Sextupole & -5.28 & $-\mathbf{6 . 4 2}$ & $-\mathbf{0 . 6 3}$ & -1.92 \\
\hline Skew Octupole & -18.51 & -21.20 & -21.18 & $-\mathbf{- 1 . 4 5}$ \\
\hline Skew Decapole & -8.52 & -4.02 & -4.23 & $-\mathbf{0 . 7 0}$ \\
\hline Skew Dodecapole & $-\mathbf{4 . 9 6}$ & $\mathbf{0 . 2 2}$ & 0.32 & -1.07 \\
\hline Skew 14-pole & 0.85 & 0.07 & -0.16 & -0.51 \\
\hline Skew 16-pole & -0.13 & -0.31 & -0.33 & -0.30 \\
\hline Skew 18-pole & 0.10 & -0.05 & -0.06 & -0.22 \\
\hline Skew 20-pole & 0.01 & 0.24 & 0.23 & 0.00 \\
\hline Skew 22-pole & 0.00 & 0.00 & 0.01 & 0.06 \\
\hline Skew 24-pole & 0.01 & -0.01 & -0.02 & -0.03 \\
\hline Skew 26-pole & 0.01 & 0.00 & 0.01 & 0.00 \\
\hline Skew 28-pole & 0.00 & 0.00 & 0.00 & 0.02 \\
\hline Skew 30-pole & 0.00 & 0.00 & 0.00 & 0.00 \\
\hline
\end{tabular}

* PMQ_005A is magnet built from magnets taken from PMQ_0005 and installed in a modified holder to reduce 12-pole

${ }^{(\dagger)}$ Magnet was measured with the magnet rotated 90 deg. about its axis, and flipped end-for-end, as compared to PMQ_005 measurements. The data were transformed in post-processing to correspond to the old orientation.

${ }^{(t+)}$ Runs 2 \& 3 are measurement in PMQ_005A with two iterations of iron shims to reduce unallowed field harmonics. (Note: Magnet name used for tesing was ERHIC-PMQ_0105 to avoid non-numeric serial number).

The shimming method is that of "floating" iron shims, operating on the principle that a narrow iron cylinder placed in an ambient magnetic field will be magnetized in the same direction as the field. Provided the field is not so high that the iron saturates (assuming mu=infinity for the iron), the magnetization will be proportional to the ambient field magnitude. The shim field contribution from the uniformly transversely magnetized iron cylinder is the same as that of a ' $\cos ($ theta)' superconducting dipole of the same dimensions: that is, an ideal external dipole field. The dipole moment is proportional to both the ambient field and the cross-sectional area of the shim. An analytic field model of these iron wires was added to PM2D and 36 of the wires were placed at 10 degree intervals around the inner bore of the magnet. The code was asked to vary the radii (areas) of the wires in order to cancel the error multipoles observed in an initial measurement of the magnet with the rotating coil. The results of this process are shown in the table below. An initial shimming designed to cancel only the sextupole was highly successful, reducing the sextupole amplitude from 20.5 units to 0.86 units, while the rest of the multipoles stayed roughly the same. It should be noted there is some logic to the shim arrangement: for the sextupole shim $(n=3)$ in a pure quadrupole background field $(m=2)$, the shim pattern has pentagonal symmetry $(n+m=5)$ and areas proportional to $1+\cos (5$ theta + phi) were used. The optimizer was used to derive a shim distribution that would cancel all observed multipoles at once. The reduction was not as dramatic as with the sextupole alone, but reduced the quadrature sum of all error multipoles from 30.4 units to 4.34 units. The rotating coil harmonics can be translated into polynomial fields with varying $x$ across the $\mathrm{y}=0$ mid-plane of the magnet, which is where the FFAG beam trajectories will be. These values are used in the figure below to calculate the relative error at any point across the aperture. Reading off the worst values at the " $53 \%$ of magnet aperture" value relevant to CBETA, this magnet had a $4.6 \mathrm{e}-3$ relative field error on the mid-plane before shimming and a 1.2e-3 relative field error after shimming. This is almost good enough for the accelerator and shimming R\&D continues to try and improve on this. Better models of saturation effects in the iron wires may help. Reading off the worst 
values at the " $53 \%$ of magnet aperture" value relevant to CBETA, this magnet had a $4.6 \mathrm{e}-3$ relative field error on the mid-plane before shimming and a 1.2e-3 relative field error after shimming. This is almost good enough for the accelerator and shimming R\&D continues to try and improve on this. Better models of saturation effects in the iron wires may help.

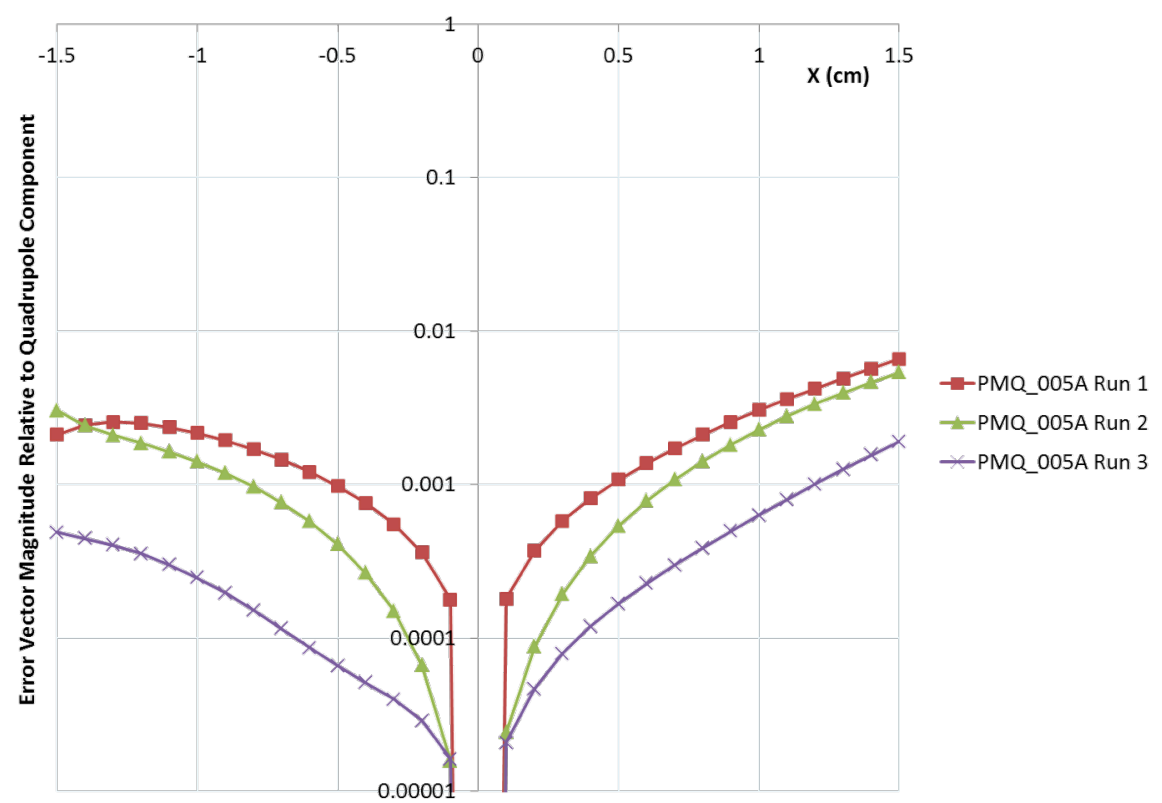

Figure 32: Relative field errors as a function of $x$ on the $y=0$ mid-plane for the shimming test magnet: before shimming (Run 1, red); after sextupole cancellation (Run 2, green) and after all-multipole shimming (Run 3, purple).

\subsection{9.a Update on Halbach Prototype Measurement Results}

During the fall of 2016 additional measurements were performed on the twelve prototype magnets. So far all twelve magnets were measured without harmonic wire corrections. All prototypes have been shimmed, five magnets were measured with shims, but there are seven magnets to be measured now. The summary of all measurements so far is shown in Fig. 15, errors in gradients are shown in Fig. 16. The stunning results for the five measured Halbach prototypes after the corrections are shown bellow in the next five tables: Table 1.9.9.2, Table 1.9.9.3, Table 1.9.9.4, Table 1.9.9.5, and the Table 1.9.9.6.

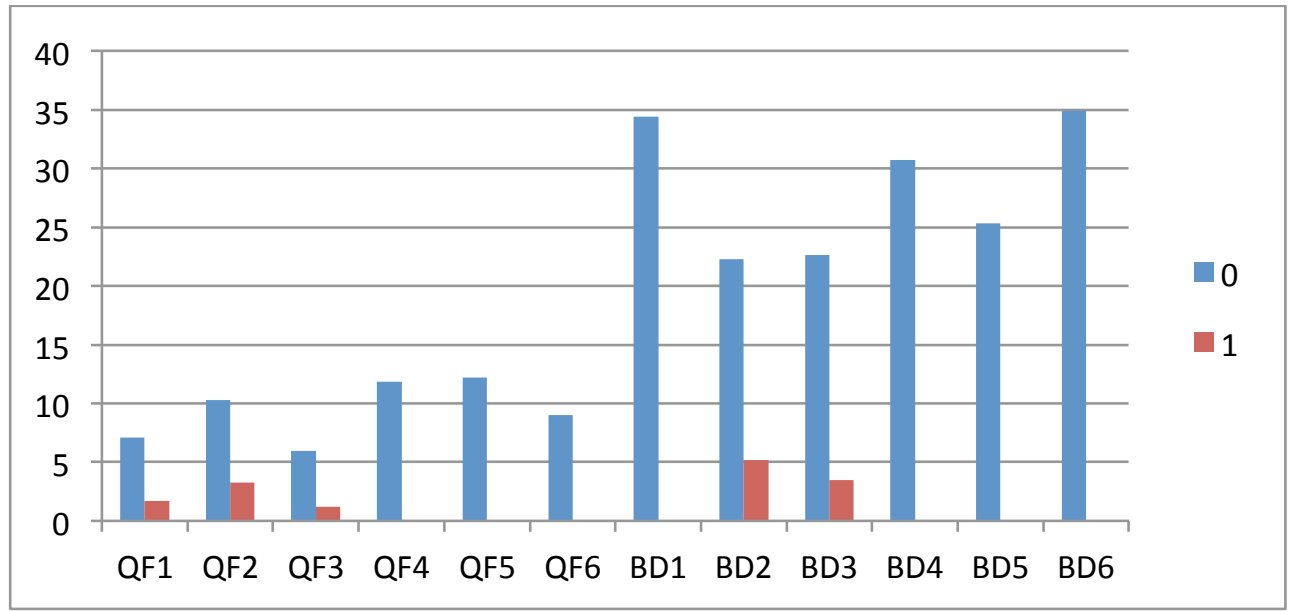

Figure 33: The ' $r m s$ ' values of the sum of all multipoles in Halbach prototype magnets before (blue color) and after the correction (red color). The x-axis shows the names of the magnets while the $y$-axis show the ' $r m s^{\prime}$ ' sum of all multipoles expressed in units (10-4 of the magnetic field at $1 \mathrm{~cm}$ radius) of each magnet. 


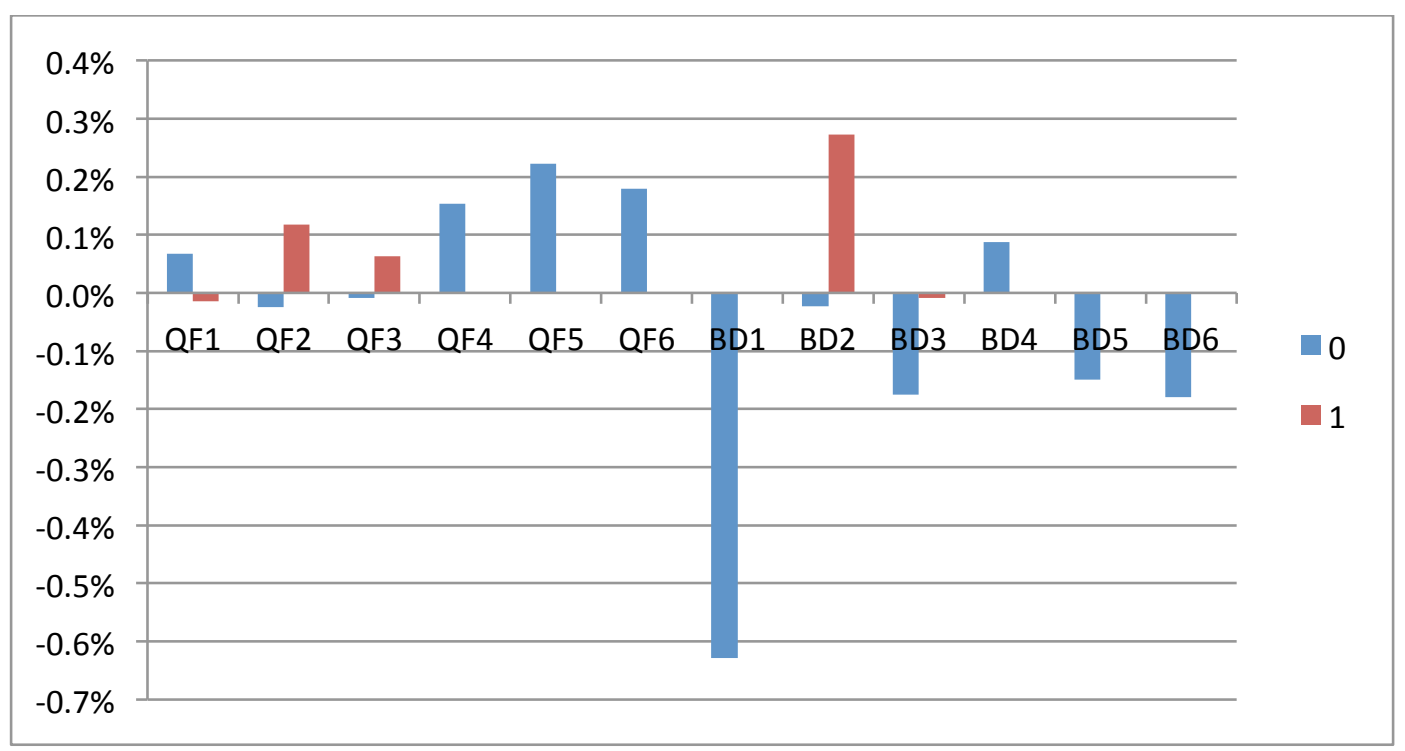

Figure 34: Errors in the integral value of the gradient in all Halbach prototype magnets before the harmonic correction (blue color) and after the correction (red color).

Table1.9.9.1: Field harmonics from ERHIC-PMQ_0501_0003_001 at R=10mm. The nominal magnet length is $57.4412 \mathrm{~mm}$ and the average field corresponds to $23.6203 \mathrm{~T} / \mathrm{m}$.

\begin{tabular}{lcc}
\hline Field harmonic & Normal units & Skew units \\
\hline Dipole & -0.00 & 0.00 \\
Quadrupole & 10000.00 & 0.00 \\
Sextupole & -0.71 & 0.34 \\
Octupole & 1.15 & 0.79 \\
Decapole & 0.11 & -0.52 \\
Dodecapole & -0.15 & 0.15 \\
14-pole & 0.04 & 0.02 \\
16-pole & -0.01 & -0.00 \\
18-pole & -0.00 & 0.00 \\
20-pole & 0.00 & 0.00 \\
22-pole & -0.00 & -0.00 \\
24-pole & -0.00 & 0.00 \\
26-pole & -0.00 & -0.00 \\
28-pole & -0.00 & 0.00 \\
30-pole & 0.00 & -0.00 \\
32-pole & 0.00 & 0.00 \\
34-pole & 0.00 & -0.00 \\
36-pole & 0.00 & -0.00 \\
38-pole & -0.00 & -0.00 \\
40-pole & -0.00 & 0.00 \\
\hline
\end{tabular}


Table1.9.9.2: Field harmonics from ERHIC-PMQ_0503_0004_001 at R=10mm. The nominal magnet length is $57.4412 \mathrm{~mm}$ and the average field corresponds to $23.6386 \mathrm{~T} / \mathrm{m}$.

\begin{tabular}{lcc}
\hline Field harmonic & Normal units & Skew units \\
\hline Dipole & 0.00 & -0.00 \\
Quadrupole & 10000.00 & 0.00 \\
Sextupole & -0.18 & -0.94 \\
Octupole & 0.34 & 0.17 \\
Decapole & -0.65 & 0.09 \\
Dodecapole & -0.05 & 0.02 \\
14-pole & -0.02 & -0.01 \\
16-pole & -0.00 & 0.00 \\
18-pole & 0.00 & 0.00 \\
20-pole & -0.00 & -0.00 \\
22-pole & -0.00 & -0.00 \\
24-pole & -0.00 & 0.00 \\
26-pole & 0.00 & -0.00 \\
28-pole & 0.00 & -0.00 \\
30-pole & -0.00 & -0.00 \\
32-pole & 0.00 & 0.00 \\
34-pole & 0.00 & 0.00 \\
36-pole & 0.00 & -0.00 \\
38-pole & 0.00 & 0.00 \\
40-pole & 0.00 & 0.00 \\
\hline
\end{tabular}

Table1.9.9.3: Field harmonics from ERHIC-PMQ_0303_0002_001 at $\mathrm{R}=10 \mathrm{~mm}$. The nominal magnet length is $61.8597 \mathrm{~mm}$ and the average field corresponds to $19.1007 \mathrm{~T} / \mathrm{m}$.

\begin{tabular}{lcc}
\hline Field harmonic & Normal units & Skew units \\
\hline Dipole & -19726.36 & 0.00 \\
Quadrupole & 10000.00 & -0.00 \\
Sextupole & -1.62 & 1.35 \\
Octupole & -2.50 & 0.85 \\
Decapole & 1.13 & -0.56 \\
Dodecapole & -0.05 & 0.22 \\
14-pole & 0.01 & 0.00 \\
16-pole & 0.03 & 0.00 \\
18-pole & -0.04 & 0.02 \\
20-pole & 0.00 & 0.01 \\
22-pole & 0.00 & -0.01 \\
24-pole & -0.00 & -0.00 \\
26-pole & -0.00 & 0.00 \\
28-pole & 0.00 & -0.00 \\
30-pole & -0.00 & -0.00 \\
\hline
\end{tabular}


Table1.9.9.4: Field harmonics from ERHIC-PMQ_0302_0003_001 at R=10mm. The nominal magnet length is $61.8597 \mathrm{~mm}$ and the average field corresponds to $19.1142 \mathrm{~T} / \mathrm{m}$.

\begin{tabular}{lcc}
\hline Field harmonic & Normal units & Skew units \\
\hline Dipole & -19712.39 & 0.00 \\
Quadrupole & 10000.00 & -0.00 \\
Sextupole & 0.52 & -1.13 \\
Octupole & -4.10 & 2.43 \\
Decapole & 1.68 & -0.92 \\
Dodecapole & -0.08 & -0.07 \\
14-pole & -0.03 & -0.05 \\
16-pole & 0.03 & -0.03 \\
18-pole & 0.00 & -0.02 \\
20-pole & 0.01 & 0.01 \\
22-pole & 0.00 & 0.00 \\
24-pole & 0.00 & -0.00 \\
26-pole & -0.00 & 0.00 \\
28-pole & 0.00 & -0.00 \\
30-pole & -0.00 & -0.00 \\
\hline
\end{tabular}

Table1.9.9.5: Field harmonics from ERHIC-PMQ_0501_0003_001 at $\mathrm{R}=10 \mathrm{~mm}$. The nominal magnet length is $57.4412 \mathrm{~mm}$ and the average field corresponds to $23.6203 \mathrm{~T} / \mathrm{m}$.

\begin{tabular}{lcc}
\hline Field harmonic & Normal units & Skew units \\
\hline Dipole & -0.00 & 0.00 \\
Quadrupole & 10000.00 & 0.00 \\
Sextupole & -0.71 & 0.34 \\
Octupole & 1.15 & 0.79 \\
Decapole & 0.11 & -0.52 \\
Dodecapole & -0.15 & 0.15 \\
14-pole & 0.04 & 0.02 \\
16-pole & -0.01 & -0.00 \\
18-pole & -0.00 & 0.00 \\
20-pole & 0.00 & 0.00 \\
22-pole & -0.00 & -0.00 \\
24-pole & -0.00 & 0.00 \\
26-pole & -0.00 & -0.00 \\
28-pole & -0.00 & 0.00 \\
30-pole & 0.00 & -0.00 \\
32-pole & 0.00 & 0.00 \\
34-pole & 0.00 & -0.00 \\
36-pole & 0.00 & -0.00 \\
38-pole & -0.00 & -0.00 \\
40-pole & -0.00 & 0.00 \\
\hline & &
\end{tabular}




\subsubsection{Measurement of the Cross Talk between two Halbach Magnets}

One of the major problems of the Hybrid-iron magnet is the cross talk between the iron belonging to neighboring magnets. A study of the magnetic field between the two Halbach magnets by the OPERA 3D program showed that the superposition theory predicts no effect of one magnet to the magnetic field within the other magnet. This was the theoretical prediction but to be completely convinced that there in no cross-talk between the Halbach magnets a measurements by use of the harmonic coil of the magnetic field inside of a single Halbach magnet with and without a presence of the neighboring magnet. Two Halbach type permanent magnets (PMQ_0001 and PMQ_0002) were assembled with a 60 $\mathrm{mm}$ distance between them with their integrated strengths of $3.775 \mathrm{~T} / \mathrm{m}$. A picture of the layout is shown in Figure 14.

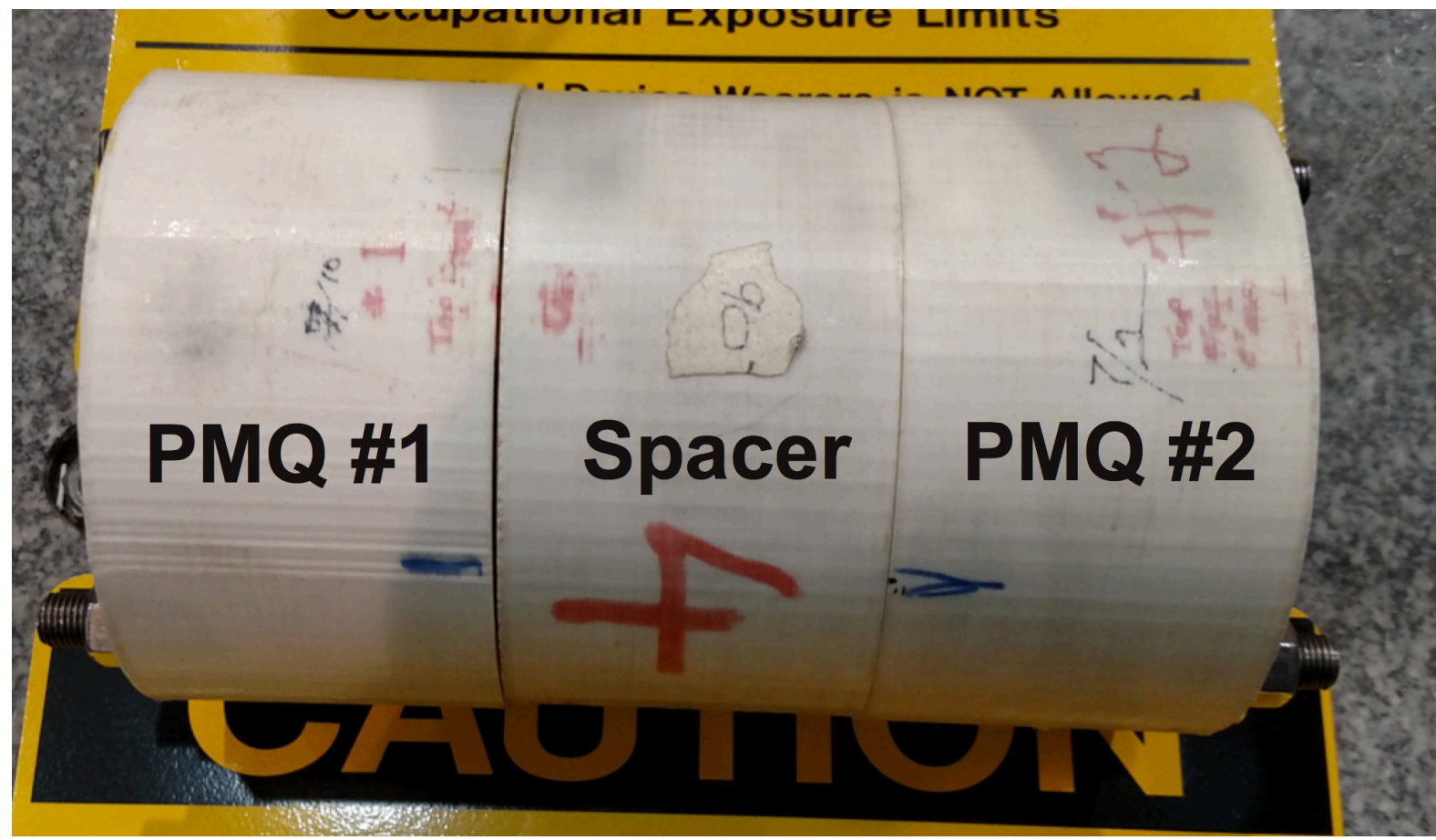

Figure 35: Layout of the set-up for measuring the cross talk between the two Halbach magnets PMQ\#1 and PMQ\#2, where the $6 \mathrm{~mm}$ long spacer made of plastic is placed between.

The results from the three types of measurements are shown in Table 13. The last column is the superposition of the two measurements. The results show that there is no measurable effect seen from one magnet to the other one. 
Table 13: Cross Talk measurement between the two Halbach Magnets

\section{Summary of field measurements in eRHIC Permanent Magnet Quadrupoles (8-Aug-2015)}

Field harmonics are absolute values in (Tesla.m) at a reference radius of $10 \mathrm{~mm}$.

Notes:

PMQ_0201 is an assembly of PMQ_0002 and PMQ_0001, with PMQ_0001 rotated 90 deg. CW. Magnets are in-line with a 60 mm gap.

PMQ_0001 was measured with the top UP. The data were reprocessed to simulate magnet rotated 90 deg. CW, as in the assembly PMQ_201

The data for individual magnets were centered and rotated, the data for the assembly are not. The comparison with superposition is not quite accurate.

\begin{tabular}{|l|c|c|c|c|}
\hline Quantity & $\begin{array}{c}\text { PMQ_0001 } \\
\text { Run 3 }\end{array}$ & $\begin{array}{c}\text { PMQ_0002 } \\
\text { Run 4 }\end{array}$ & $\begin{array}{c}\text { PMQ_0201 } \\
\text { Run 1 }\end{array}$ & $\begin{array}{c}\text { PMQ_0201 } \\
\text { (Superposition) }\end{array}$ \\
\hline Integrated Gradient (T) & -1.8656 & 1.9098 & 0.0416 & 0.0442 \\
\hline Normal Dipole & -- & -- & $-3.082 \mathrm{E}-04$ & -- \\
\hline Normal Quadrupole & $-1.866 \mathrm{E}-02$ & $1.910 \mathrm{E}-02$ & $3.999 \mathrm{E}-04$ & $4.421 \mathrm{E}-04$ \\
\hline Normal Sextupole & $-3.57 \mathrm{E}-05$ & $-5.50 \mathrm{E}-05$ & $-8.81 \mathrm{E}-05$ & $-9.07 \mathrm{E}-05$ \\
\hline Normal Octupole & $7.94 \mathrm{E}-06$ & $-3.91 \mathrm{E}-06$ & $7.42 \mathrm{E}-06$ & $4.04 \mathrm{E}-06$ \\
\hline Normal Decapole & $1.55 \mathrm{E}-05$ & $-9.99 \mathrm{E}-06$ & $3.52 \mathrm{E}-05$ & $5.54 \mathrm{E}-06$ \\
\hline Normal Dodecapole & $3.53 \mathrm{E}-04$ & $-3.74 \mathrm{E}-04$ & $-3.02 \mathrm{E}-05$ & $-2.09 \mathrm{E}-05$ \\
\hline Normal 14-pole & $4.01 \mathrm{E}-06$ & $6.24 \mathrm{E}-07$ & $4.66 \mathrm{E}-06$ & $4.63 \mathrm{E}-06$ \\
\hline Normal 16-pole & $-7.23 \mathrm{E}-07$ & $-3.97 \mathrm{E}-07$ & $-6.28 \mathrm{E}-07$ & $-1.12 \mathrm{E}-06$ \\
\hline Normal 18-pole & $-9.67 \mathrm{E}-09$ & $4.33 \mathrm{E}-07$ & $1.33 \mathrm{E}-06$ & $4.23 \mathrm{E}-07$ \\
\hline Normal 20-pole & $4.34 \mathrm{E}-06$ & $-5.52 \mathrm{E}-06$ & $-1.25 \mathrm{E}-06$ & $-1.18 \mathrm{E}-06$ \\
\hline Normal 22-pole & $-4.00 \mathrm{E}-08$ & $3.84 \mathrm{E}-08$ & $-1.55 \mathrm{E}-08$ & $-1.60 \mathrm{E}-09$ \\
\hline Normal 24-pole & $1.52 \mathrm{E}-08$ & $1.05 \mathrm{E}-08$ & $1.83 \mathrm{E}-08$ & $2.57 \mathrm{E}-08$ \\
\hline Normal 26-pole & $-1.92 \mathrm{E}-08$ & $-2.00 \mathrm{E}-08$ & $-9.18 \mathrm{E}-08$ & $-3.93 \mathrm{E}-08$ \\
\hline Normal 28-pole & $-1.82 \mathrm{E}-07$ & $2.32 \mathrm{E}-07$ & $5.50 \mathrm{E}-08$ & $5.10 \mathrm{E}-08$ \\
\hline Normal 30-pole & $-5.61 \mathrm{E}-09$ & $1.69 \mathrm{E}-09$ & $-1.36 \mathrm{E}-09$ & $-3.92 \mathrm{E}-09$ \\
\hline
\end{tabular}

\begin{tabular}{|l|c|c|c|c|}
\hline Quantity & $\begin{array}{c}\text { PMQ_0001 } \\
\text { Run 3 }\end{array}$ & $\begin{array}{c}\text { PMQ_0002 } \\
\text { Run 4 }\end{array}$ & $\begin{array}{c}\text { PMQ_0201 } \\
\text { Run 1 }\end{array}$ & $\begin{array}{c}\text { PMQ_0201 } \\
\text { (Superposition) }\end{array}$ \\
\hline Field Angle (mr) & -- & -- & -- & -- \\
\hline Skew Dipole & -- & -- & $8.730 \mathrm{E}-04$ & -- \\
\hline Skew Quadrupole & -- & -- & $1.156 \mathrm{E}-04$ & -- \\
\hline Skew Sextupole & $-4.71 \mathrm{E}-05$ & $3.06 \mathrm{E}-06$ & $-4.34 \mathrm{E}-05$ & $-4.40 \mathrm{E}-05$ \\
\hline Skew Octupole & $-2.03 \mathrm{E}-05$ & $2.10 \mathrm{E}-06$ & $-1.59 \mathrm{E}-06$ & $-1.82 \mathrm{E}-05$ \\
\hline Skew Decapole & $-1.54 \mathrm{E}-05$ & $-1.52 \mathrm{E}-05$ & $-9.36 \mathrm{E}-05$ & $-3.07 \mathrm{E}-05$ \\
\hline Skew Dodecapole & $3.79 \mathrm{E}-06$ & $-1.40 \mathrm{E}-06$ & $-1.21 \mathrm{E}-05$ & $2.39 \mathrm{E}-06$ \\
\hline Skew 14-pole & $2.69 \mathrm{E}-06$ & $3.05 \mathrm{E}-07$ & $2.52 \mathrm{E}-06$ & $2.99 \mathrm{E}-06$ \\
\hline Skew 16-pole & $-4.69 \mathrm{E}-07$ & $-2.32 \mathrm{E}-07$ & $2.98 \mathrm{E}-07$ & $-7.01 \mathrm{E}-07$ \\
\hline Skew 18-pole & $-3.81 \mathrm{E}-07$ & $3.11 \mathrm{E}-07$ & $-2.35 \mathrm{E}-06$ & $-6.96 \mathrm{E}-08$ \\
\hline Skew 20-pole & $-1.04 \mathrm{E}-07$ & $1.20 \mathrm{E}-08$ & $-3.08 \mathrm{E}-07$ & $-9.23 \mathrm{E}-08$ \\
\hline Skew 22-pole & $-7.02 \mathrm{E}-08$ & $7.72 \mathrm{E}-08$ & $3.89 \mathrm{E}-08$ & $7.00 \mathrm{E}-09$ \\
\hline Skew 24-pole & $3.71 \mathrm{E}-08$ & $-7.72 \mathrm{E}-09$ & $-5.25 \mathrm{E}-08$ & $2.94 \mathrm{E}-08$ \\
\hline Skew 26-pole & $1.68 \mathrm{E}-08$ & $-1.47 \mathrm{E}-08$ & $1.65 \mathrm{E}-07$ & $2.05 \mathrm{E}-09$ \\
\hline Skew 28-pole & $5.75 \mathrm{E}-09$ & $-7.60 \mathrm{E}-09$ & $1.32 \mathrm{E}-08$ & $-1.85 \mathrm{E}-09$ \\
\hline Skew 30-pole & $2.43 \mathrm{E}-09$ & $-1.52 \mathrm{E}-09$ & $-1.99 \mathrm{E}-09$ & $9.05 \mathrm{E}-10$ \\
\hline
\end{tabular}

\subsubsection{Plan for BNL CBETA Halbach Prototypes (April 2016)}

Purpose-built prototype magnets for CBETA have also been ordered, including the "lopsided Halbach" magnet $B D$. Due to the 2-3 month magnet lead times, these are from an old lattice design Cell_Brooks_2015-12-11 rather than the most recent Cell_Smoothpipe_2016-02-04 presented in this report, but they are similar. Delivery of permanent magnet pieces should occur at the end of March 2016. A comparison of the magnets in the two versions is given in the table below.

Table 1.9.11.1: Comparison of current lattice Halbach magnets to those of the CBETA prototypes ordered.

\begin{tabular}{|l|c|c|c|c|}
\hline Parameter & QF current & QF prototype & BD current & BD prototype \\
\hline Length & $96.3 \mathrm{~mm}$ & $114.9 \mathrm{~mm}$ & $126.4 \mathrm{~mm}$ & $123.7 \mathrm{~mm}$ \\
\hline Gradient & $-28.80 \mathrm{~T} / \mathrm{m}$ & $-23.62 \mathrm{~T} / \mathrm{m}$ & $19.19 \mathrm{~T} / \mathrm{m}$ & $19.12 \mathrm{~T} / \mathrm{m}$ \\
\hline Dipole at center & 0 & 0 & $-0.2680 \mathrm{~T}$ & $-0.3768 \mathrm{~T}$ \\
\hline Max good field radius & $19.5 \mathrm{~mm}$ & $20.2 \mathrm{~mm}$ & $19.5 \mathrm{~mm}$ & $13.7 \mathrm{~mm}$ \\
\hline Min inner radius & $36.5 \mathrm{~mm}$ & $37.2 \mathrm{~mm}$ & $36.5 \mathrm{~mm}$ & $30.7 \mathrm{~mm}$ \\
\hline Max outer radius & $70.2 \mathrm{~mm}$ & $62.4 \mathrm{~mm}$ & $69.3 \mathrm{~mm}$ & $59.4 \mathrm{~mm}$ \\
\hline $\begin{array}{l}\text { Max field in good field } \\
\text { region }\end{array}$ & $0.56 \mathrm{~T}$ & $0.48 \mathrm{~T}$ & $0.64 \mathrm{~T}$ & $0.64 \mathrm{~T}$ \\
\hline Max field at “pole tip" & $1.05 \mathrm{~T}$ & $0.88 \mathrm{~T}$ & $0.97 \mathrm{~T}$ & $0.96 \mathrm{~T}$ \\
\hline
\end{tabular}


These have been ordered from 'AllStar Magnetics' rather than Shin-Etsu (due to cost reasons), which mean a larger magnetization angle error in the blocks of \pm 5 degrees as specified by their factory. Shimming methods will be tested to see if they can compensate for this larger error, possibly including shimming magnets instead of the iron wires.

\subsubsection{Manufacturing Pipeline and Vendors}

Discussions are starting with magnet manufacturing companies about what they can build for CBETA. The pipeline of magnet manufacture and assembly onto the machine breaks down into the four stages below.

\subsubsection{2.a Permanent Magnet Wedges}

These will be purchased, directly or indirectly, from a company. As mentioned previously, Shin-Etsu Corporation is a large manufacturer of the permanent magnet blocks with reasonably high quality. AllStar Magnetics has also provided BNL permanent magnets block in the past (for instance the radiation damage experiment), although they specify larger tolerances on their magnetization angles. Electron Energy Corporation (EEC) has recently succeeded in an SBIR proposal for CBETA and eRHIC magnet development worth $\sim 1 M$ but this is spread over three years from April 2016 to April 2019. EEC manufactures both the blocks and magnet assemblies on-site in their machine shop. Finally, Holger Witte has contacted 'VacuumSchmelze' GmbH for magnet blocks for the iron-poled quadrupole. Other companies not contacted yet include the undulator manufacturer KYMA.

Of these companies, AllStar generally provides the lowest cost but the least accurate magnetization vector guarantee ( \pm 5 degrees). Shin-Etsu provides \pm 1 degree tolerance with some additional cost for tooling. EEC say even \pm 0.5 degrees is possible but there is an associated cost since additional steps of demagnetizing the block, re-grinding it to an accurate shape and re-magnetizing it have to occur

\subsubsection{2.b Magnet Assembly}

Although in theory this could be done on the BNL or Cornell sites, it seems that several companies are willing to bid for this work and are capable of doing it. EEC could be used as an end-to-end vendor for these first two steps. RADIABEAM LLC will make assemblies and girders but have to get the PM blocks from another company. They previously gave a cost estimate for assembling the CBETA magnets and girders and are the only ones to have significant accelerator field experience (in fact they also make Halbach magnets for electron microscopes). Their absolute tolerances on positioning magnets on the girders were $0.1 \mathrm{~mm}$.

\subsubsection{2.c Shimming and Rotating Coil Measurements}

Discussions so far with magnet manufacturers are indicating that the rotating coil is a specialized piece of measurement equipment for accelerator applications. None of the companies contacted so far have functioning rotating coils, although RADIABEAM and EEC have Hall probes for field mapping. The shimming method works best using a rotating coil, so this stage is likely to be done in the BNL magnet division, where they have done it before. 


\subsubsection{2.d Alignment and Girder}

Since the magnets will need to be removed to do separate rotating coil and shimming steps, a fully integrated manufacture (measurement while on girder) does not look possible. Instead, survey fittings will be included in the non-magnetic body during the magnet assembly step and these will be used in the hall at Cornell to fit in with their on-site survey system. The survey references may also be used in the rotating coil stage to ensure alignment between the magnetic field and the magnet holder.

\subsection{Response to Magnet reviewers' Objections from March 2016 review}

The Magnet Review Committee in March 2016 was given an assignment to make a choice between the hybrid-iron-dominated magnets or Halbach type of magnets. The members of the Committee were: Steve Peggs (BNL), Michael Anerella (BNL), Michael Harrison (BNL), Vladimir Kashikhin (FNAL), Jim Clarke (Daresbury), Sasha Temnykh (Cornell), Bruce Brown (FNAL), Toshiya Tanabe (BNL), John Seeman (SLAC), Mauricio L. Lopes (JLAB), David Douglas (JLAB), Animesh Jain (BNL at the time), and Dave Harding (FNAL). The next section contains comments of each member of the committee with answers (yellow overlay) to all objections to the Halbach magnets.

- Steve Peggs and Michael Anerella (BNL) firmly selected the hybrid-iron-dominated magnets using arguments of established technology, temperature compensation included, full existing design and ease in production with reduced risks for 220 magnets, and an existing expertise in iron magnet technology. Halbach magnets are said to require temperature compensation, require very tight tolerances in the permanent magnet production, and represent more technical challenge with commensurate risks. Answers: to the objections from the two reviewers with respect to the Halbach magnets is that the 150 $\mathrm{MeV}$ twelve Halbach prototype magnets have shown that the technical challenges were resolved and the risks are reduced as the results from the magnet measurements will show (the largest gradient error was $0.2 \%$, with the largest magnet multipole error up to the $20^{\text {th }}$ pole was less than a unit $10^{-4}$ ).

- Mike Harrison (BNL) conclusion was that both magnets can be built but are not optimal. His complaint was that hybrid-iron-dominated magnets are too wide and he made a suggestion to reduce the maximum energy of $E_{\max }=200 \mathrm{MeV}$ if they are to be used. The Halbach magnet has appeal because it is smaller and has no crosstalk but the corrector is too large and produces too much heat. -Answers: The design with the maximum energy of $E_{\max }=250 \mathrm{MeV}$, analyzed by Mike Harrison did have large corrector size $30 \mathrm{~cm}$ and corrector coils required water-cooling. For the CBETA base line lattice with the maximum energy of $150 \mathrm{MeV}$ every dimension shrinks according to $250 \rightarrow 150 \mathrm{MeV}$ reduction of the BRHO. In addition for the gradient correction of $\Delta G / G \leq \pm 2 \%$ the $1.5 \mathrm{~cm}$ thick windings have the current density of $1 \mathrm{~A} / \mathrm{mm}^{2}$, while the measured limit for water cooling requirement is $2 \mathrm{~A} / \mathrm{mm}^{2}$ (the $\Delta G / G \leq \pm 2 \%$ ) was obtained by multiplying by 10 the maximum value of the gradient error from the twelve built prototype magnets of $0.2 \% \times 10=2 \%$ ). The cross talk between the magnets does not exists was shown not only by the 3D OPERA magnetic field superposition demonstration but by direct harmonic coil measurements of the two magnets separated longitudinally by $6 \mathrm{~cm}$.

- Vladimir Kashikhin (BNL) has selected the hybrid-iron-dominated magnet, citing that the magnetic field is more predictable by using ferromagnetic poles, while reported Halbach's model confirmed the issue with the proper magnetization orientation. -Answers: to the Vladimir Kashikhin legitimate concern for the Halbach magnet and proper magnetization required are the measured twelve $250 \mathrm{MeV}$ 
prototypes shown bellow in this report. In addition the Harmonic coil measurements have shown very good agreement between the OPERA 3D predictions.

- Jim Clark (Daresbury) solution is to use Electro Magnets (EM) instead. The hybrid-iron-dominated magnet: the proposed windings for the various corrections are quite complex. The positioning of the iron poles is crucial for the field quality; the design seems to rely on dead reckoning with no adjustment possible. From experience I would say that this is difficult to get right. If shims have to be used they are labor intensive. The $200 \mathrm{MeV}$ option uses $5 \mathrm{~A} / \mathrm{mm}^{2}$ windings. This is more than many pure (EM) quads we have built. This goes back to my original query about comparison against EM quad?

Halbach magnets: Although, there is no crosstalk between the PM assemblies, what about with the iron correctors that surround the PM? The BD magnet is innovative but looks awkward to assemble accurately, but it might be possible to include correctors in this space instead of outside. Again the coils of the corrector (fig 7) look quite significant, is it really worth

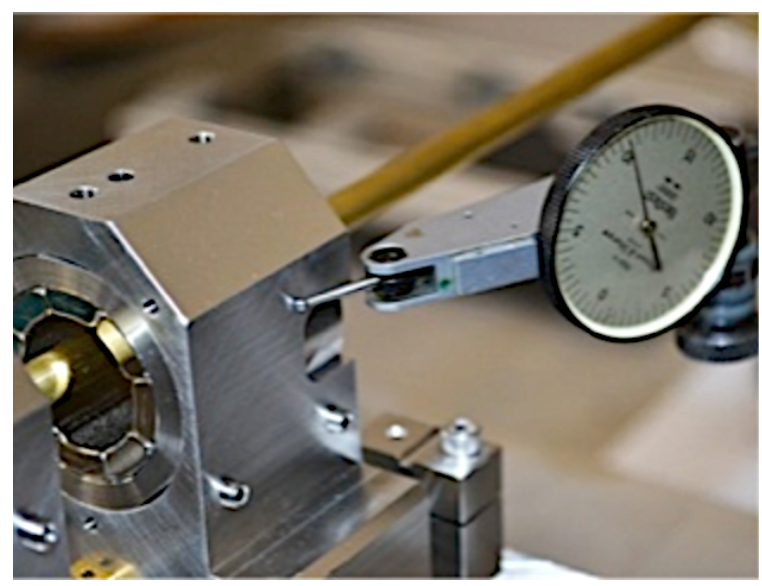
using PM? The wire shimming appears to be quite complex \& labor intensive. - An answer: to the legitimate Jim Clark concern of the corrector iron frame influence to the magnetic field inside the Halbach magnet comes from the 3D OPERA field simulation with and without the corrector as the relative permeability of the Halbach permanent magnet material is not exactly equal to 1 bur $\mu_{\text {rel }}=1.05$ 1.1. Not only the predictions by OPERA program were used, but Animesh Jain harmonic coil measurements (shown in the attachment) with and without the corrector frame around the Halbach magnet showed the same confirmation. The effect of the dipole corrector and the iron frame to the quadrupole gradient does exist and it is $0.2 \%$. The wire shimming is complex and the labor is required for two Harmonic measurements: one before the correction and one after the correction. As the results from the first Harmonic coil measurements are obtained, it requires one minute of time to use the program analyses written by Stephen Brooks to obtain the results for which correction wire and it length needs to go to what position. The correction wires are placed manually and the total time required for that is definitely longer than a minute but has already been developed with a detail procedure established.

- Sasha Temnykh (Cornell) selected the hybrid-iron dominated magnet. The Halbach magnets are much more complicated than the second hybrid-iron-dominated magnets. Their manufacturing would require fabrication of much larger number various types of components. That will increase significantly probability of errors, production risk, time and cost in comparison with second type. Taking into account number of various types of PM blocks in the Halbach magnet and unavoidable errors in magnetization and in dimensions, one can expect more difficulties in procedure of magnetic field tuning. Again that will drive up production time and cost. - Answers: Legitimate concerns of Sasha Temnykh for the large number of various types of components so far did not show to be a serious problem, at least in the $250 \mathrm{MeV}$ Halbach prototype magnet productions. We have also contacted few other companies ("Electron Energy Corporation-EEC" in Pennsylvania and "RADIABEAM" in California) and obtained answers that they had already build large number of specialty permanent Halbach type magnets without serious problems as shown in Figure 36 on the right built by RADIABEAM similar to our design. In addition the estimated cost of the Halbach

magnets with respect the hybrid-iron-dominated magnets is at least $\$ 1 \mathrm{M}$ less.

- Bruce Brown (Fermilab) I am not prepared to make a strong recommendation. I do believe you are likely to achieve your goals with the 'hybrid' (iron dominated) design. Only the cross talk between nearby magnets is a new issue. The iron wire shim is a neat trick. Be sure that the wires cannot rust 
during the life of the project. It is worth remembering an abandon idea from the Recycler thermal compensation story. You have considered using water to stabilize the temperature. I would think that the water alone would be good enough but the thermal pipe idea is so neat that I decided to mention it. - Answers: One of the suggestions by Bruce Brown is to make sure that the correction wires could rust and we will try to resolve this issue.

- Toshiya Tanabe (BNL) - It appears that simulation used single value of 1.025 for the permeability of $\mathrm{NdFeB}$ magnet. To be more precise, one must use two different values for easy and hard directions. At the NSLS-II undulator, we use $\mu_{E A S Y}=1.05$ and $\mu_{\text {HARD }}=1.15$. That's why you don't get exact superposition of fields in higher order multipoles when PM magnet and Window Frame magnets are combined. Answers: This time the answers to the concerns are written immediately following each. This is a real legitimate concern and we had paid serious attention to it. Animesh Jain did confirm by the harmonic coil measurement with a single Halbach magnet, with the unpowered corrector frame, and with powered corrector frame the effect mentioned above and this is shown in the Appendix. As already mentioned above the effect is $\Delta G / G \leq \pm 0.2 \%$. For the new magnets we need to perform at least one measurement of both Halbach magnets with and without the corrector frame, without and with powering the corrector windings to calibrate the effect to what is the required size for correction.

-Since PPM quad has magnetic material close to the beam pipe, the demagnetization by irradiation must be avoided by using PM with high intrinsic coercivity. 22 kOe does not seem to be conservative enough. -Answers: This legitimate concern is accepted and a new material is used for design with the permanent $\mathrm{N} 35 \mathrm{UH}, \mathrm{B}_{\mathrm{R}}=1.17-1.21 \mathrm{~T}, \mathrm{H}_{\mathrm{c}}(\mathrm{KOe})=10.8-11.4, \mathrm{H}_{\mathrm{Cl}}(\mathrm{KOe})>25, \mathrm{BH}_{\max }(\mathrm{MGOe})=33-35, \mathrm{~T}_{\text {MAx }}\left(\mathrm{C}^{\circ}\right) \leq 180$, where UH means more resistant to demagnetization and radiation damage. A difference in price due to this request is $\$ 174,756.30$ to $\$ 240,678.90$.

-Yoke material of the Window Frame corrector is not specified in the report. Even though PM quads have minimum magnetic interaction and WF magnet is known to have better stray field characteristics, these WF magnets in closely place condition may create interaction with adjacent ones. -Answers: This concern is quite appropriate. We have studied with OPERA 3D effect of five magnets with corrector frames with correctors on in one direction or opposite directions as shown in the Nick Tsoupas report. The effect is present but it is very small.

-I also concur to use NdFeB magnets instead of SmCo ones. However the argument "a radiation test has shown NdFeB of an appropriate grade survives $>100$ Gy of radiation" cannot ensure the selected grade is adequate unless the same magnetic circuit instead of a single magnet was irradiated. -Answers: We followed the advice and the $250 \mathrm{MeV}$ prototype magnets as well as the future $150 \mathrm{MeV}$ are built with $\mathrm{NdFeB}$ magnetic material, but with a reasonable magnetization $\mathrm{B}_{\mathrm{R}} \approx 1.17-1.21$.

- It is recommended that higher quality magnet (from 'Shin-etsu') be used instead of cheaper alternatives. - Answers: We have built twelve magnets with ALSTAR-MAGNETICS (http://allstarmagnetics.com) and so far did not see any problem with the permanent magnet material. A difference in cost is $50 \%$ and our budget is very limited.

- 2\% correction ability with Panofsky coils may not be enough. A part of this correction might be consumed to compensate temperature fluctuation. - Answers: As stated above we have tested twelve prototype Halbach magnets and the largest difference with respect to the expected gradient is $\Delta G / G \leq$ $\pm 0.2 \%$. It has been decided to use 10 times the value $\Delta G / G \leq \pm 2 \%$, mostly due to our limitation on the current density through the correction coils of $>100 \mathrm{~A} / \mathrm{cm}^{2}$.

My recommendation: provided that the vacuum chamber can be designed without much difficulty, I

recommend, "Iron dominated magnet solution" due to the following reasons:

1) Easy correction capability with EM coils.

2) Interaction issues appear to have been solved. 
3) Risk of PM demagnetization is smaller because of larger distance from the beam pipe compared to Halbach design.

4) If it is really necessary, temperature compensation scheme can be incorporated.

Answers: The Halbach magnet comparison to the Hybrid-iron-magnets so far shows few not negligible advantages with respect to the Hybrid-iron-dominated magnets: first is the cost reduction, no interaction issues, simpler, smaller size, simple correction system, round vacuum chamber, dramatically smaller weight, excellent magnetic field quality (after the correction), etc. Following advice of the permanent magnet selection reduced the risk of demagnetization " 3 )".

- John Seeman (SLAC) - Halbach magnets: Permanent magnet quadrupoles should work well in this application. - The plan is to correct the field after assembly. A real time field-measuring fixture is needed to allow a human to fix the harmonics in an hour or so. Answers: The time for the magnetic field correction is explained above in answer to Jim Calk concerns. To repeat: the computer program analysis of the harmonic coil rough results and calculation of the required wire size and placement corrections is less than a minute and placement of the wires into the already built frames shown bellow is definitely less than 10 minutes. To speed the assembly process, pre-measurements of the blocks for go or no-go with appropriate tolerances should be done.

Answer: This is a very good advice we will include it into the procedure.

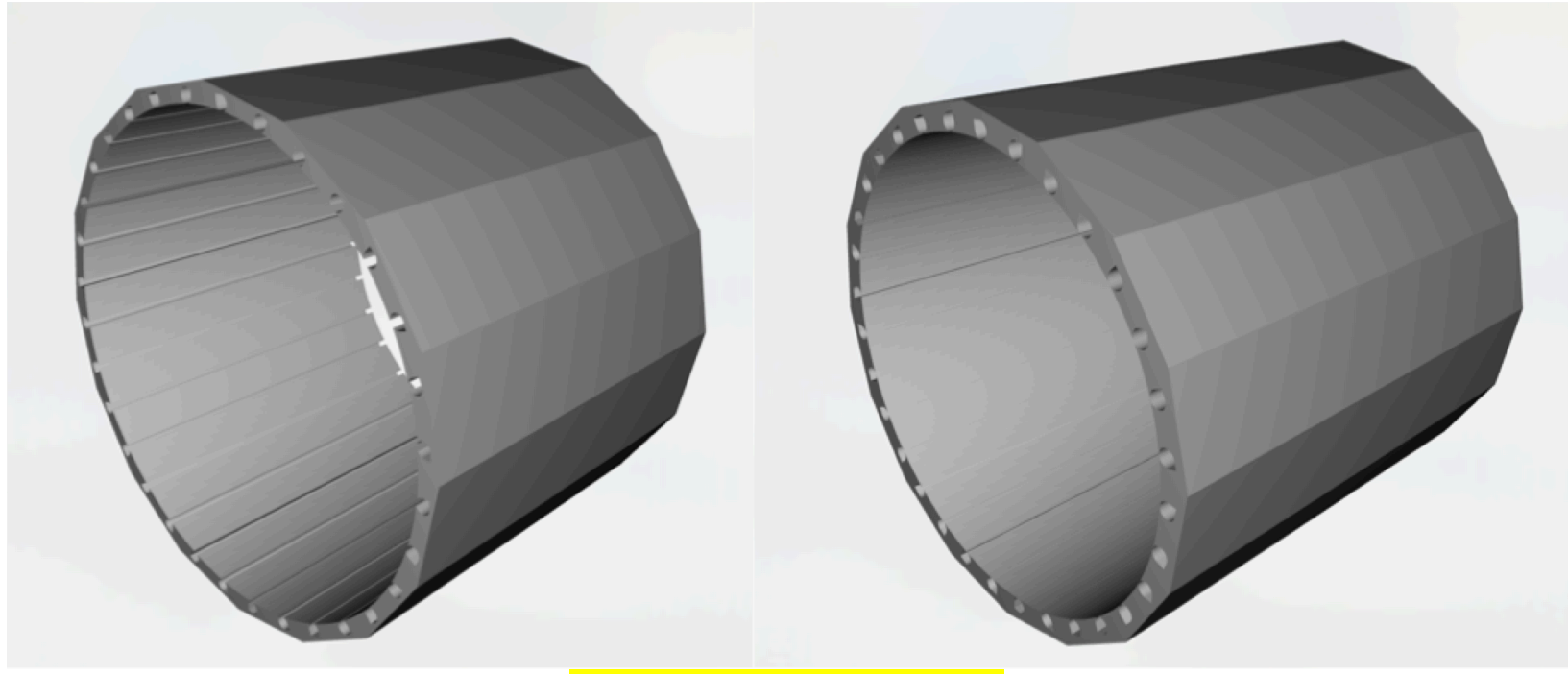

Figure 37: Frames for correctors

For the PEP-II IR PM quadrupoles we adjusted the PM blocks as we assembled them so no shimming was needed after assembly. Field levels to $10^{-4}$ were corrected over 4 inch bores. Please see attached photos. This took extra work upfront but the magnets were excellent. Pre-adjustments could be done for CBETA too if needed. An alternative is to do what we did at PEP-II where we had adjacent counter rotating PM quadrupole rings that can be adjusted to give varying quadrupole strengths up to +/$100 \%$. Just split your PM magnets in half longitudinally and add rotators. These can also be a skew 
quadrupole correction. Please see the PEP-II version in the attached photograph in Fig. 38. The nearly straight vacuum chamber associated with this design is a big advantage for construction and beam instabilities. The Panofsky quadrupole corrector is very weak and takes up a lot of space. The window frame Panofsky quad is not very efficient as a lot of flux goes outward away from the bore of the magnet (equal magnetic path lengths).

- Iron magnets: The design as presented should work. The temperature compensation is a good idea but reduces the field strengths. It would be good to not have to do the additional quadrupole coils on the poles. Stamped laminations for the steel could reduce costs. The assembly of the magnetized blocks into the steel frames will involve many forces and, thus, a strong assembly fixture will need to be made. There are trim coils on each magnet pole to provide adjustability. Why not go to fully powered quadrupoles with only water-cooled coils and forget the PM blocks altogether. The magnets would probably be smaller, cheaper, and more accurate, but cost more to run. In the sketches of the Fe magnets the sides are split at all quadrants. In practice only a top-bottom or left-right split is needed. Fewer splits will save costs and reduce overall assembly errors. The vacuum chamber design will be quite complicated and will likely have beam instability issues from HOMs, SR heating, resistive wall.

-Overall: The effects of the energy spread of each of the beam-lets should be calculated and shown from the simulations. Is there any dispersive dilution to the effective emittance and are sextupole terms needed? Given the various constraints, it is likely that the optimum quadrupole spacing in the lattice will depend on either the permanent magnet (PM) or iron type quadrupole specifically, e.g. magnetic cross talk, field strengths, tolerances, and will need to be specifically chosen before the design is frozen. One further option is to make all the QD quadrupoles permanent magnets (symmetrical ones) and all the QF quadrupoles made from fully powered steel. Thus, the cross talk is solved, Panofsky quads for the PMs are not needed, there is plenty of adjustability, the temperature variation of the PM magnet can corrected in the adjacent magnet, and the vacuum chambers would be much straighter than for steel only.

The choice of the magnet type for CBETA should be the same technology as expected to be used for the design of eRHIC. Otherwise, it is not testing the right solution even though it may not be the best choice for CBETA. The answer to this question lays also on the cost of each magnet. So far the results indicate that the required permanent material volume is smaller for Halbach type and the total price is significantly lower.

- Mauricio L. Lopes (JLAB) - Iron Magnets In this document the author provides a good description of the magnet, listing materials and their properties, proportions and dimensions. The design is based on previous design of magnets that were built and operational for a long time. The parameters related to Electromagnetic Analysis were well defined. The problem of the cross talk between adjacent magnets has been discussed. However, due to the lack of clear specifications it is hard to know if this is even a problem. The field uniformity of the magnet is achieved by shaping the low carbon iron poles. This is done by the means of stamped laminations, which are known for its high accuracy. Differences among iron batches can be minimized by shuffling the laminations after they are stamped. The claimed field uniformity

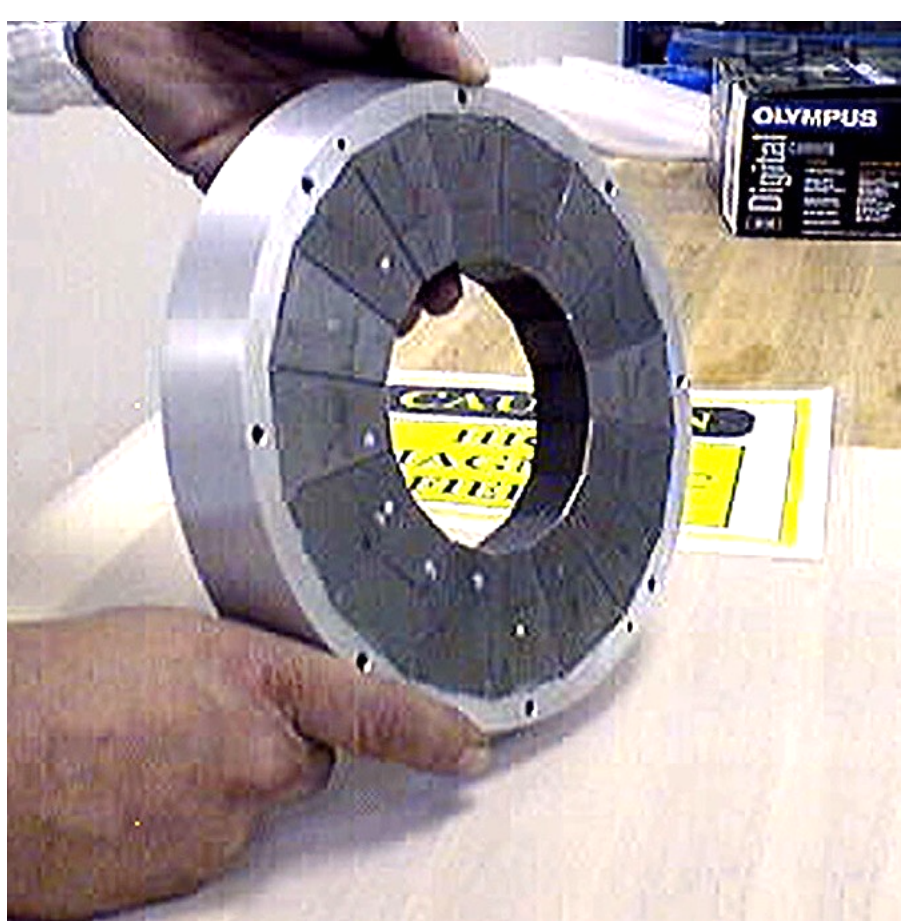


$(0.6 \%)$ is easily achievable by this method. The PMs will be made of simple shaped blocks of NdFeB. This should be fairly easy to be procured and the tolerances for those blocks are fairly achievable. The problem of field dependence with temperature was properly addressed and the proposed solution is based on a past proven concept. It involves the use of NiFe strips in between blocks of $\mathrm{NdFeB}$ (proportion of 20 to $80 \%$ ); this method provides compensation between 20 to $30{ }^{\circ} \mathrm{C}$. Correction coils can provide some tuning between 5 and $10 \%$ if necessary without any change in field quality. However, depending on the level of correction, water cooled coils may be required. Extra simple winding can provide additional corrections like normal and skew dipole and skew quadrupole. These windings do not create a larger magnet footprint. Once again, it was not clear what level of fields is needed. A concept for the mechanical assembly of these magnets was presented and it looks reasonable.

- Halbach Magnets In this document some specs are presented. It makes hard to compare because the specs seem to contradictory with the other design. Two concepts of classical Halbach magnets are presented. Both magnets are made of 16 segments with the magnetization of each block properly oriented to give the expected field. The PM material will be the same NdFeB. No passive compensation due to variation of the temperature was proposed. Instead it is intended to use the correction coils. The correction coils are located on an external frame around the main Halbach magnet. This increases the magnet volume and its footprint. This may or may not be a concern since there is no clear specification for the dimensions of the magnet. The author present the results of eRHIC PM quadrupole magnetic measures without shimming. It shows the changes in the high order harmonics but it is not clear what the acceptable levels are. Field quality can be improved by shimming through the insertion of iron wires in different positions of the inner bore. This can be challenging if the number of magnets are larger. A response to this concern is provided above in previous answers to Jim Clark and John Simon.

Conclusions and Recommendations

-First and foremost a clear set of requirements regardless of the magnetic design approach. This should include but not limited to: maximum external dimensions, field requirements and field quality, number of magnets of each kind, etc. A difference in size between the Halbach magnets with the frame corrector outside is $23.3 \mathrm{~cm}$ with respect to the outside dimensions of the Hybrid-iron-dominated magnet $66 \mathrm{~cm}$.

-Both magnets would benefit from a Perturbation Analysis in order to identify the weak spots in their designs.

-The iron magnet design report seems to be in a more advance stage than the Halbach. Halbach needs to develop a mechanical assembly model. A full mechanical model drawings and assembly as well as disassembly procedure is developed and are shown in this report.

-Plans for thermal compensation were presented in both cases, however the Halbach magnet did not show evidence that the method in fact works without penalizing the field quality. In the mechanical assembly the water-cooling system is added and shown.

-Halbach magnet PM parts will be more difficult to procure than the iron dominate magnets. Depending on the total number of magnets this can be an issue for manufacturing. The procurement of the permanent magnet for the twelve $250 \mathrm{MeV}$ Halbach prototype magnets as well as for the new $150 \mathrm{MeV}$ does not represent any problem. The delivery time is 80 days.

-Iron Magnets field quality will depend on the quality of its laminations, which are usually very precise. This can be an advantage if a large number of magnets will be produced. The PM has very little influence in the field quality. Usually Iron magnets do not have the necessity of being individually shimmed after construction.

-Halbach magnets have the field quality depending exclusively on the quality of the machine of the PM. The field quality can be achieved by individually shimming each magnet. Once again, this may be 
challenging depending of the number of magnets being produced. The answer to this question has been already explained above in answers to John Simon and Jim Clark.

-Iron magnet seems to be more versatile ("one design fits them all") than the Halbach design.

If possible purse with full-scale prototype of both magnets. This did happened already with the Halbach twelve prototype magnets. One good quality Hybrid-iron dominated magnet has also been built and measured.

-David Douglas (JLAB) - I went through the magnet comparison you sent week before last \& concur in detail with content and conclusions. A couple things that don't seem to be addressed either in the document and the design:

1. There has not been a single high power CW ERL ever operated that didn't end up putting in some aberration compensation in (via sextupoles or higher order...) - either to fix transverse aberrations (nonlinear dispersion or dispersive effects that couple to orbit offsets - T126, T226, ... aberrations) or to linearize the longitudinal phase space so as to manage energy spread after recovery.

2. There seems to be an underlying assumption that the iron quads will give more stable fields of better quality. I am concerned this implicitly assumes the remnant field of the iron can be neglected and/or will be homogeneous. I suspect this will not be the case: the yokes will carry the history of their assembly (with PM material), handling, and installation (especially being placed in close proximity to one another). It is entirely possible that this will differentially magnetize the yokes. Moreover, "significant" trims - at the $10-20 \%$ level - will generate hysteresis effects that may (or may not) render reproducibility (and potentially - in nearby magnets - field quality) problematic.

I would thus encourage use of the Halbach magnets - with the caveat that they be carefully temperature controlled. -Yes, this comment has been taken seriously and the water-cooling system, as already mentioned, is a part of the design.

Though there will be more scatter in the fields and field quality, they will be more controllable in the sense that they are less likely to be changed in a random manner than are magnets that involve (unsaturated and unstandardized) iron. Thus, what you fabricate and measure is what you install; there is risk (potentially considerable) that this is not the case for the iron magnets. Moreover, the Halbach magnets will allow straightforward and reproducible implementation of both linear (and nonlinear should that prove necessary later on) corrections: all required multipoles can be imposed from outside, just like the dipole and quadrupole corrections. -This comment is correct and the results from the Halbach prototype measurement confirm superb quality of the magnetic field after the corrections.

Though some range of nonlinear control can be had by asymmetrically driving an iron quad, this also moves the field center around, further coupling the already challenging task of orbit correction and optics tuning, and, of course, stability (over the course of prolonged tuning) and reproducibility is a serious challenge when only limited trims are made available on the iron quads.

I hope this is of some help. I got lattice information from Chris \& will be taking a look at transverse/longitudinal coupling and error sensitivities in the next several days. I'll keep you posted. Safe travels southward!

-Animesh Jain (formerly BNL now ANL)

-Halbach magnet design

-The correction of quadrupole field strength variation due to temperature variation and magnet-tomagnet variation would be done using quadrupole correctors outside the permanent magnet quadrupole. The stated strength of $\pm 0.45 \mathrm{~T} / \mathrm{m} \times 0.11 \mathrm{~m}$ amounts to about $\pm 1.8 \%$ correction only (for QF at $28.8 \mathrm{~T} / \mathrm{m} \times 0.0963 \mathrm{~m}$ ). It should be verified if this strength would be sufficient. For example, the variation in integrated gradient of the 5 Halbach quadrupoles mentioned in the report was $\pm 1.2 \%$. Also, even this small correction requires a current density of $3.75 \mathrm{~A} / \mathrm{mm}^{2}$, which implies water-cooled coils. 
This would increase the complexity and cost of the system. -Answer: Excellent comments and correct concerns. Adding the water-cooling system attached to the aluminum frame around the permanent magnet material, and allowing $\pm 2 \%$ in gradient correction with less than $100 \mathrm{~A} / \mathrm{cm}^{2}$ without requirement for the water-cooling of the correction coils, have solved this problem.

It is a good idea to circulate the cooling water on the outside of the quadrupoles in order to minimize the field variations due to temperature, but there could still be issues due to non-uniform temperature in the magnet (e.g. a radial temperature gradient) unless suitable materials with good thermal conductivity are used. This also adds to the complexity of the magnet. - Answer: yes the water-cooling system is attached to the aluminum $3 / 8$ frame surrounds the permanent magnet material.

-Ability to tune the field quality using floating iron shims should allow the required field quality to be achieved without much added cost. Answer: Yes this has been really achieved for all twelve Halbach 250 $\mathrm{MeV}$ prototype magnets.

-Cross talk between two corrector magnets should be studied, although this may not be a serious issue if the corrector fields are small. -Answer: Yes this was studied first by 3D opera field as well with the measurements you had performed after this review.

A conceptual design for the BD magnet is presented using an asymmetric distribution of permanent magnets. This is a clever way to optimize the use of magnet material, and also allows use of a smooth beam pipe. But the complexity of manufacturing such an object should be looked at. - Answer: The complexity did not present a serious problem during the twelve prototype Halbach magnet production and the detail procedure was established as shown is Figure 5.

-"Iron Dominated Magnets for CBETA". The design uses simple permanent magnet geometry and benefits from experience at other laboratories.

- Field quality is governed by the poles, and good magnet-to-magnet control can be achieved with stamped laminations. But use of iron poles also makes it difficult to produce fields of good quality for other multipolarities (e.g. dipole correction).

-The quadrupole correction coils around the steel poles could produce correction strength comparable to the Panofsky quad described in the Halbach design option with air-cooled coils (current density 1 $\mathrm{A} / \mathrm{mm}^{2}$ ). But these coils consume some axial space beyond the steel poles. Current densities higher than $\sim 1 \mathrm{~A} / \mathrm{mm}^{2}$ would require cooling (either using chill plates, or direct cooling of hollow conductor).

-Design of the dipole and skew quad correctors is complicated, and these will not be so easy to produce (as compared to the simple window frame design).

-Since both Qf and Qd are combined function magnets, it may be possible to further optimize the magnet design by introducing left-right asymmetry in the pole shapes and/or the placement of driving permanent magnets, as is done, for example, for the BD magnet described in the Halbach quadrupole report. This may also reduce the complexity of the beam tube.

Summary: Both the Halbach type and iron dominated options have been studied in detail, but both options still require some more studies, as pointed out in the report above. At present, more test results are available for the Halbach option, but the iron-dominated option is based on past experience and test results in a prototype are also likely to be available soon. It would be prudent to wait until the prototype tests of both options are completed, the designs have matured more (e.g. BD design and stronger correctors for the Halbach option, and dipole correctors for the iron dominated option), and a better estimate of relative costs is available.

If a choice must be made now, I would mildly favor the Halbach option for the following reasons: 
-Although the PM material may be more expensive, the assembly of the magnets would be much simpler, and perhaps cheaper. Answer: It has been shown that the volume required for the Halbach magnets is smaller than the one for the Hybrid-iron-dominated magnets and the cost of the permanent magnet material is smaller for the Halbach magnets.

-Very simple geometry for the dipole and quadrupole correctors. Also, the manufacturing of correctors and the quads can be completely delinked, as these are two independent units. Answer: That is correct.

-Practically no interaction issues between quadrupoles, although interaction between the correctors needs to be studied. Answer: This has already been discussed above.

-Least amount of axial and transverse space needed.

-Ability to easily tweak higher harmonics on a magnet-to-magnet basis, thus potentially relaxing the assembly tolerances. Answer: correct.

-The iron dominated design option should be "low risk" in terms of field quality, but it does not appear to be "low cost" overall. The design of dipole correctors is very preliminary at this stage, and is likely to be complex. There are also issues with the shape of the beam pipe, and issues related to the use of stainless steel pipe.

The above choice assumes that the remaining design issues in the Halbach design option can be easily resolved and the proximity of permanent magnets to the beam does not present any radiation damage issues.

-David Harding (Fermilab) C-beta sounds like an excellent application for permanent magnets. I look forward to hearing more about the project as it develops.

Either design can be expected to require trimming of individual magnets. This is not onerous if you plan for it and define a standard procedure and algorithms, as we demonstrated with the Recycler.

The requirement to be able to split the magnet and reassemble it is going to be very difficult for either style. I would try very hard to avoid having to split the magnet, regardless of the design choice. If necessary, then it must be planned for, building into the magnet yoke or support structure appropriate attachment points that the tooling can grab. Answer: quite correct concern, as it is very critical to connect with a very high precision the upper with the lower Halbach magnet part. The problem has been solved with a very simple procedure but it added an additional step in the assembly procedure and an additional assembly frame to be used for both sides of the magnets.

If you are looking for more vendors to assemble magnets, Hi-Tech Manufacturing, LLC, of Schiller park, IL, (http://www.hi-tech-mfg.com/) has assembled permanent magnets for both Argonne (undulators) and Fermilab (some post-Recycler dipoles). In our case, we provided the design and the SmCo5 bricks and $\mathrm{H}$-Tech fabricated or procured all the other components, designed the tooling, and assembled the magnets. We did the QC on the incoming bricks and measured the completed magnets. From the writeups, I don't understand why beam tubes are so different between the two magnet styles. Passive compensation of temperature variations is very attractive from an operations perspective. (The original Recycler design had one set of quads with a temperature stability requirement that we could not meet with the NiFe compensator. We stabilized the temperature with heaters that kept the magnets above the nominal tunnel temperature. This led to a to a rapid loss of field strength in the strontium ferrite magnetic bricks.) Under Iron Risks, I see "Beam pipe magnetization affects field quality (due to stainless steel pipes)". With proper specifications and QC, that should not be an issue. I couldn't find any details 
about the mechanical structure imagines for the Halbach magnets. While excellent for prototyping, I don't expect the 3-D printer will produce a rad-hard support system. Perhaps the nests would be machined from aluminum blocks? Answer: this might be quite possible but it will introduce additional cost in a very limited budget constrains.

I would be more concerned about the reproducibility of the field after splitting and reassembly with the Halbach style than with the iron, but that's partly based on not seeing a Halbach mechanical concept.

-Answer: The Halbach magnet design does allow assembly and disassembly of both upper and lower parts with a very high precision. This was accomplished with an additional frame-fixture used for each Halbach magnet which allows by creation of the connecting holes to be used later for precise assembly as shown later in Figure 8.

In the iron-dominated design, it would be worth looking again at the choice between laminations and solid cores. The materials might be less expensive for the laminations, but there would be increased complexity and increased labor. As is noted in the iron-dominated note, the density of the magnetic bricks can be increased. We have not found it necessary to leave space for anything except temperature compensation. 


\section{COST ESTIMATES}

There are 6 columns shown in Table 1. The first column on the left side represents the HYBRID-IRON magnets cost estimated by the Superconducting Magnet Department (SMD) at BNL, the second column is so far the lowest sale quote obtained from the DANFYSIK, one of five outside companies outside of the BNL. These estimates include 120 focusing and 120 defocusing magnets. The major items in the ironhybrid magnet in the first column, starting with the lowest position, are: 1. Order laminations: $\$ 660,000.0,2$. Order permanent magnet blocks: $\$ 1,100,000.00,3$. Order back-leg magnet steel: $\$ 880,000.00$, 4. Order machined aluminum components: $\$ 660,000.00,5$. Order magnet hardware: $\$ 220,000.00$ (color navy-blue), 6. Order nickel-iron for temperature compensation: $\$ 110,000.00,7$. Assemble magnets with correctors: $\$ 428,000.00,8$. Measure and adjust magnets: $\$ 142,000.00$ (beigeorange), 9. Measure and Adjust magnets: $\$ 63,514.00$ (blue color) 10 . Survey magnets during testing at the girder: $\$ 71,368.00$ (brown) 11. Survey magnets during testing: $\$ 31,757.00$ (green-color), 12 . Order Correctors: $\$ 440,000.00$ (pink-blue color), 13. Order girders: $\$ 84,000.00$ (beige-color), Girder design and procurement: $\$ 33,376.00$ (brown color), 14. Assemble girders with vacuum pipes $\$ 36,332.00$ (green), 15. Survey the girders: $\$ 36,000.00$ (purple color), 16. Shipping the girders: $\$ 10,500.00$ (beige color), 17 . Survey the magnet/girders assembled: $\$ 9083.00$ (green color), 18. Survey magnet/girder assemblies: $\$ 4041.00$ (beige color).

TABLE 1: COST ESTIMATES FOR HYBRID IRON and HALABCH MAGENTS

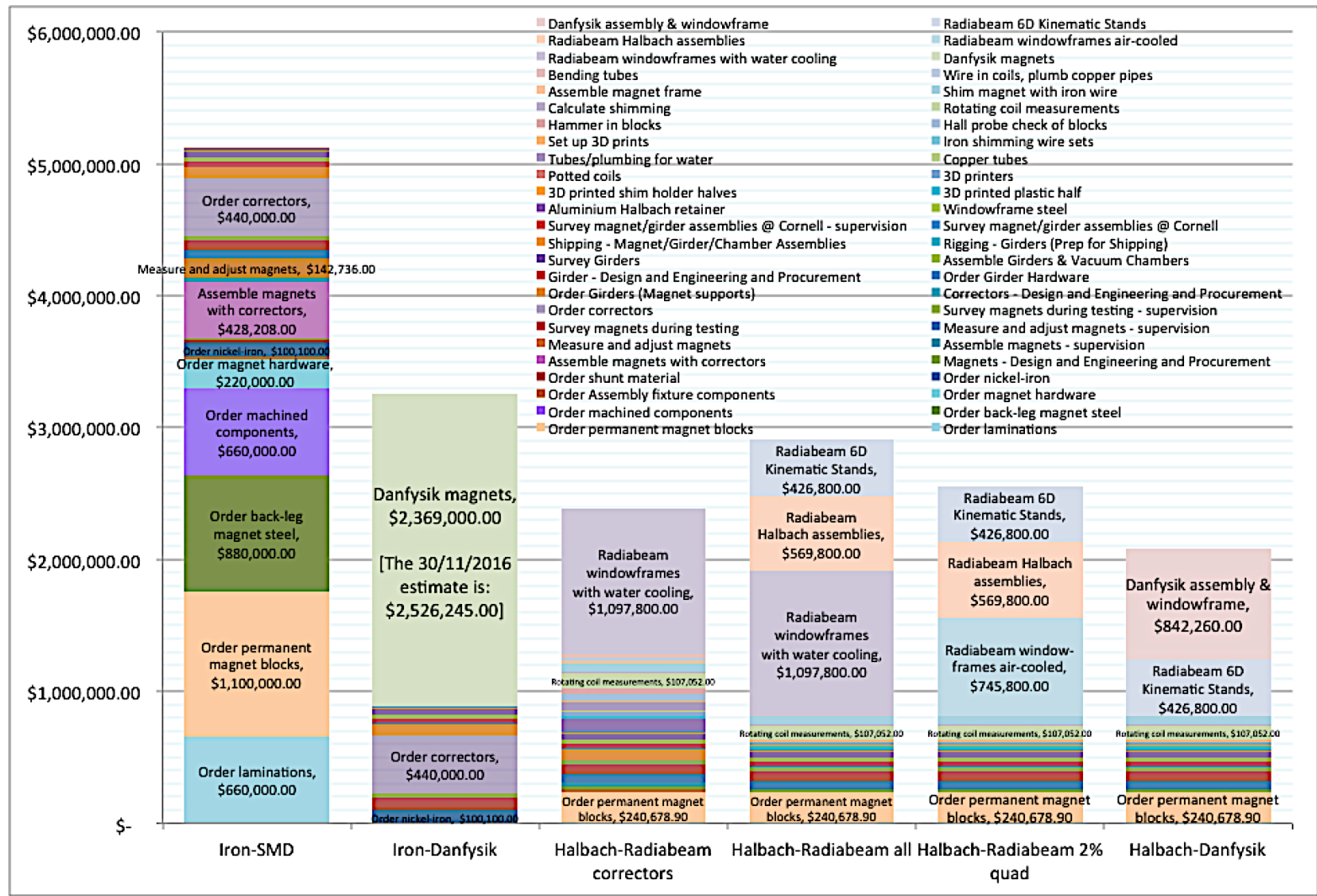

A detail explanation of the third column describing the Halbach magnet production starts with the lowest block: 1 . Order permanent magnet material: $\$ 240,678.90$ (beige color). This cost estimate corresponds to the sales quote dated October 28, 2016, from the same company "Allstar Magnetics" previously providing material for 12 Halbach prototype magnets already built. A bit more advanced 
Table 3: Cost of the FFAG Magnets

\begin{tabular}{|c|c|c|c|c|c|c|c|}
\hline & Iron-SMD & Iron-Danfysik & $\begin{array}{l}\text { Halbach- } \\
\text { Radiabeam } \\
\text { correctors }\end{array}$ & $\begin{array}{l}\text { Halbach- } \\
\text { Radiabeam all }\end{array}$ & $\begin{array}{l}\text { Halbach- } \\
\text { Radiabeam 2\% } \\
\text { quad }\end{array}$ & $\begin{array}{l}\text { Halbach- } \\
\text { Danfysik }\end{array}$ & $\begin{array}{l}\text { Halbach-no } \\
\text { chiller plates } \\
\text { (old) }\end{array}$ \\
\hline Order laminations & $\$ 660,000.00$ & & & & & & \\
\hline Order permanent magnet blocks & $\$ 1,100,000.00$ & & $\$ 240,678.90$ & $\$ 240,678.90$ & $\$ 240,678.90$ & $\$ 240,678.90$ & $\$ 240,678.90$ \\
\hline Order back-leg magnet steel & $\$ 880,000.00$ & & & & & & \\
\hline Order machined components & $\$ 660,000.00$ & & & & & & \\
\hline Order magnet hardware & $\$ 220,000.00$ & & & & & & \\
\hline Order Assembly fixture components & $\$ 20,020.00$ & & $\$ 20,020.00$ & & & & $\$ 20,020.00$ \\
\hline Order nickel-iron & $\$ 100,100.00$ & $\$ 100,100.00$ & & & & & \\
\hline Order shunt material & $\$ 22,000.00$ & $\$ 22,000.00$ & & & & & \\
\hline $\begin{array}{l}\text { Magnets - Design and Engineering } \\
\text { and Procurement }\end{array}$ & $\$ 16,688.00$ & & $\$ 16,688.00$ & $\$ 16,688.00$ & $\$ 16,688.00$ & $\$ 16,688.00$ & $\$ 16,688.00$ \\
\hline Assemble magnets with correctors & $\$ 428,208.00$ & & & & & & \\
\hline Assemble magnets - supervision & $\$ 31,757.00$ & & $\$ 31,757.00$ & & & & $\$ 31,757.00$ \\
\hline Measure and adjust magnets & $\$ 142,736.00$ & & & & & & \\
\hline Measure\&adj. magnets - supervision & $\$ 63,514.00$ & & $\$ 63,514.00$ & $\$ 63,514.00$ & $\$ 63,514.00$ & $\$ 63,514.00$ & $\$ 63,514.00$ \\
\hline Survey magnets during testing & $\$ 71,368.00$ & $\$ 71,368.00$ & $\$ 71,368.00$ & $\$ 71,368.00$ & $\$ 71,368.00$ & $\$ 71,368.00$ & $\$ 71,368.00$ \\
\hline $\begin{array}{l}\text { Survey magnets during testing - } \\
\text { supervision }\end{array}$ & $\$ 31,757.00$ & $\$ 31,757.00$ & $\$ 31,757.00$ & $\$ 31,757.00$ & $\$ 31,757.00$ & $\$ 31,757.00$ & $\$ 31,757.00$ \\
\hline Order correctors & $\$ 440,000.00$ & $\$ 440,000.00$ & & & & & \\
\hline $\begin{array}{l}\text { Correctors - Design and Engineering } \\
\text { and Procurement }\end{array}$ & $\$ 2,086.00$ & $\$ 2,086.00$ & $\$ 2,086.00$ & $\$ 2,086.00$ & $\$ 2,086.00$ & $\$ 2,086.00$ & $\$ 2,086.00$ \\
\hline Order Girders (Magnet supports) & $\$ 84,000.00$ & $\$ 84,000.00$ & $\$ 84,000.00$ & & & & $\$ 84,000.00$ \\
\hline Order Girder Hardware & $\$ 5,600.00$ & $\$ 5,600.00$ & $\$ 5,600.00$ & $\$ 5,600.00$ & $\$ 5,600.00$ & $\$ 5,600.00$ & $\$ 5,600.00$ \\
\hline $\begin{array}{l}\text { Girder - Design and Engineering and } \\
\text { Procurement }\end{array}$ & $\$ 33,376.00$ & $\$ 33,376.00$ & $\$ 33,376.00$ & $\$ 33,376.00$ & $\$ 33,376.00$ & $\$ 33,376.00$ & $\$ 33,376.00$ \\
\hline $\begin{array}{l}\text { Assemble Girders \& Vacuum } \\
\text { Chambers }\end{array}$ & $\$ 36,332.80$ & $\$ 36,332.80$ & $\$ 36,332.80$ & $\$ 36,332.80$ & $\$ 36,332.80$ & $\$ 36,332.80$ & $\$ 36,332.80$ \\
\hline Survey Girders & $\$ 36,332.80$ & $\$ 36,332.80$ & $\$ 36,332.80$ & $\$ 36,332.80$ & $\$ 36,332.80$ & $\$ 36,332.80$ & $\$ 36,332.80$ \\
\hline Rigging - Girders (Prep for Shipping) & $\$ 3,177.72$ & $\$ 3,177.72$ & $\$ 3,177.72$ & $\$ 3,177.72$ & $\$ 3,177.72$ & $\$ 3,177.72$ & $\$ 3,177.72$ \\
\hline $\begin{array}{l}\text { Shipping - Magnet/Girder/Chamber } \\
\text { Assemblies }\end{array}$ & $\$ 10,500.00$ & $\$ 10,500.00$ & $\$ 10,500.00$ & $\$ 10,500.00$ & $\$ 10,500.00$ & $\$ 10,500.00$ & $\$ 10,500.00$ \\
\hline $\begin{array}{l}\text { Survey magnet/girder assemblies @ } \\
\text { Cornell }\end{array}$ & $\$ 9,083.20$ & $\$ 9,083.20$ & $\$ 9,083.20$ & $\$ 9,083.20$ & $\$ 9,083.20$ & $\$ 9,083.20$ & $\$ 9,083.20$ \\
\hline $\begin{array}{l}\text { Survey magnet/girder assemblies @ } \\
\text { Cornell - supervision }\end{array}$ & $\$ 4,041.80$ & $\$ 4,041.80$ & $\$ 4,041.80$ & $\$ 4,041.80$ & $\$ 4,041.80$ & $\$ 4,041.80$ & $\$ 4,041.80$ \\
\hline Window frame steel & & & & & & & $\$ 88,000.00$ \\
\hline Aluminum Halbach retainer & & & $\$ 88,000.00$ & & & & $\$ 88,000.00$ \\
\hline 3D printed plastic half & & & $\$ 22,000.00$ & $\$ 22,000.00$ & $\$ 22,000.00$ & $\$ 22,000.00$ & $\$ 22,000.00$ \\
\hline 3D printed shim holder halves & & & $\$ 4,400.00$ & $\$ 4,400.00$ & $\$ 4,400.00$ & $\$ 4,400.00$ & $\$ 4,400.00$ \\
\hline $3 \mathrm{D}$ printers & & & $\$ 25,000.00$ & $\$ 25,000.00$ & $\$ 25,000.00$ & $\$ 25,000.00$ & $\$ 25,000.00$ \\
\hline Potted coils & & & & & & & $\$ 440,000.00$ \\
\hline Copper tubes & & & $\$ 11,000.00$ & & & & $\$ 11,000.00$ \\
\hline Tubes/plumbing for water & & & $\$ 66,000.00$ & & & & $\$ 66,000.00$ \\
\hline Iron shimming wire sets & & & $\$ 1,001.73$ & $\$ 1,001.73$ & $\$ 1,001.73$ & $\$ 1,001.73$ & $\$ 1,001.73$ \\
\hline Set up 3D prints & & & $\$ 15,878.50$ & $\$ 15,878.50$ & $\$ 15,878.50$ & $\$ 15,878.50$ & $\$ 15,878.50$ \\
\hline Hall probe check of blocks & & & $\$ 47,635.50$ & & & & $\$ 47,635.50$ \\
\hline Hammer in blocks & & & $\$ 45,892.00$ & & & & $\$ 45,892.00$ \\
\hline Rotating coil measurements & & & $\$ 107,052.00$ & $\$ 107,052.00$ & $\$ 107,052.00$ & $\$ 107,052.00$ & $\$ 107,052.00$ \\
\hline Calculate shimming & & & $\$ 10,585.67$ & $\$ 10,585.67$ & $\$ 10,585.67$ & $\$ 10,585.67$ & $\$ 10,585.67$ \\
\hline Shim magnet with iron wire & & & $\$ 63,514.00$ & $\$ 63,514.00$ & $\$ 63,514.00$ & $\$ 63,514.00$ & $\$ 63,514.00$ \\
\hline Assemble magnet frame & & & $\$ 22,946.00$ & & & & $\$ 22,946.00$ \\
\hline Wire in coils, plumb copper pipes & & & $\$ 22,946.00$ & & & & $\$ 22,946.00$ \\
\hline Bending tubes & & & $\$ 35,684.00$ & & & & $\$ 35,684.00$ \\
\hline Danfysik magnets & & $\$ 2,369,000.00$ & & & & & \\
\hline \multicolumn{2}{|c|}{ Radiabeam windowframes with water cooling } & & $\$ 1,097,800.00$ & $\$ 1,097,800.00$ & & & \\
\hline Radiabeam windowframes air-cooled & & & & & $\$ 745,800.00$ & & \\
\hline Radiabeam Halbach assemblies & & & & $\$ 569,800.00$ & $\$ 569,800.00$ & & \\
\hline Radiabeam 6D Kinematic Stands & & & & $\$ 426,800.00$ & $\$ 426,800.00$ & $\$ 426,800.00$ & \\
\hline Danfysik assembly \& window frame & & & & & & $\$ 842,260.00$ & \\
\hline Total & $\$ 5,112,678.32$ & $\$ 3,258,755.32$ & $\$ 2,387,648.62$ & $\$ 2,908,368.12$ & $\$ 2,556,368.12$ & $\$ 2,083,028.12$ & $\$ 1,817,848.62$ \\
\hline
\end{tabular}


permanent magnet material $\mathrm{N} 35 \mathrm{SH}$ instead of $\mathrm{M}-90111-\mathrm{M}$ is selected, as it is more resistant to radiation and demagnetization. 2. Design and engineering procurement: $\$ 16,688.00$ (green color). 3. Measure and adjust Halbach magnets, estimate from the company RADIABEAM, $\$ 63,514.00$ (blue color), 4 . Survey magnets during testing: $\$ 71,368.00$ (brown color), estimate from the company RADIABEAM, 5.Survey magnets during testing, estimate from the company Radiabeam: $\$ 31,757.00$ (green color), 6 . Order Girder hardware $\$ 5,600.00$ (blue color) estimate from the company RADIABEAM (blue), 7. Girder design engineering/procurement: $\$ 33,736.00$ (brown color), estimate from the company RADIABEAM, 8 . Assemble girders and vacuum chambers: $\$ 36,332.80$ (green) estimate from the company RADIABEAM, 9. Survey girders: $\$ 36,000.00$, estimated by RADIABEAM, (purple color), 10 . Shipping magnet, girder and assembly: $\$ 10,500.00$, estimated by RADIABEAM, (color orange), 11 . Survey magnets on the girders: $\$ 9,083.02$, estimated by RADIABEM (blue color), 12 . Survey magnet/girder assembly at Cornell University: $\$ 4,041.80$ estimated by Cornell (color brown), 13. Aluminum Halbach retainer: $\$ 88,000.00$ (color purple), 14. 3D printer plastic parts made at BNL: $\$ 22,000.00$ (color light-blue), 15. 3D printer shim holder half's: $\$ 4400.00,16$. At BNL: 3D printers: $\$ 25,000.00$ (color darker-blue), 16. Copper tubes for water-cooling: $\$ 11,000.00$ (green color), 17 . Copper tubes pluming for water-cooling: $\$ 66,000.00$ (color purple-light), 18. Set-up 3D printers: $\$ 15,875.50$ (color orange), 19. Hall probes check of each block: $\$ 47,635.50$, estimate from RADIABEAM (color blue-light), 20. Placement of the blocks and gluing into the aluminum frames: $\$ 45,892.00$ (color beige-orange), 21 . Rotating coils measurements at BNL: $\$ 107,052.00$ (color green), 22. Calculation of the corrections after the first Harmonic measurements: $\$ 10,585.70$ (color pink), 23. Shimming the iron wires into the blocks: $\$ 63,514.00$ (color blue), 24. Assemble magnet frame: $\$ 22,946.00$ (color beige-orange), 25 . Copper pipe plumbing: $\$ 22,946.00$ (color blue), 26. Cooper pipes bending: $\$ 35,684.00$ (color beige-orange), 27: 'RADIABEAM' window frame with water-cooling: $\$ 1,097,800.00$ (color light-purple), or 'RADIABEAM' window corrector frame air-cooled $\$ 745,800.00$ (color light-blue).

The lowest sale quotes are obtained from the company DANFYSIK for both kinds of magnets as shown in second column for the Hybrid-iron and in the seven columns for the Halbach magnets, respectively. The total cost of the Halbach magnet, with quadrupole corrector range of $\pm 2 \%$, is $\$ 2,083,028.12$, while the lowest price of the Hybrid-iron magnet is $\$ 3,258,755.32$. A Difference in price is $\$ 1,175,727.20$, as shown bellow in Table 3.

A difference in between the lowest estimated cost of the Hybrid-iron magnet of $\$ 3,258,755.32$ and the Halbach magnet of $\$ 2,083,028.12$ is equal to $\$ 1,175,727.20$. In the case of CBETA limited budget this represents significant savings.

\section{RISKS AND MITIGATIONS (Stephen Brooks)}

\subsection{Technological principles and challenges}

The Halbach magnet design produces magnetic field directly from magnetized blocks, without intervening iron to shape the field. This traditionally has made it difficult to get good field quality, as the field will be directly sensitive to the blocks' positions, angles and magnetization strength, the last of which can vary by of the order of $1 \%$ from the factory.

The shimmed Halbach design proposed here circumvents these difficulties by producing the field in two stages. The permanent magnets are placed (without sorting) and produce a field with errors of the 
order of $1 \%$, but the errors are repeatable provided the blocks are fixed in place. This un-shimmed magnet is then measured on a rotating coil to determine the error harmonics. A combination of iron wires of various sizes placed around the inner bore has been found to cancel any combination of multipoles provided they are weak enough. The picture below shows 32 such wires confined in channels in a 3D printed plastic shim holder.

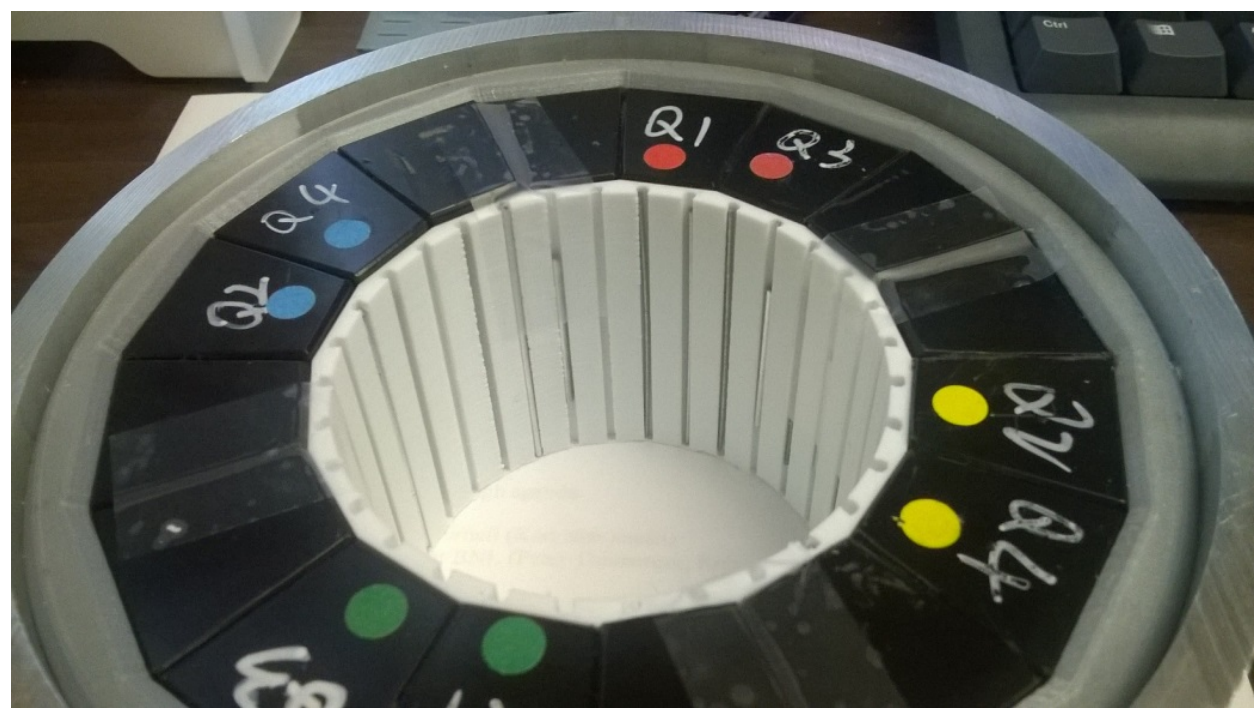

Figure 39: The above magnet is part of a prototype series of 6 quadrupole and 6 combined-function magnets, which have been shimmed to field quality required for CBETA, as described in other documents.

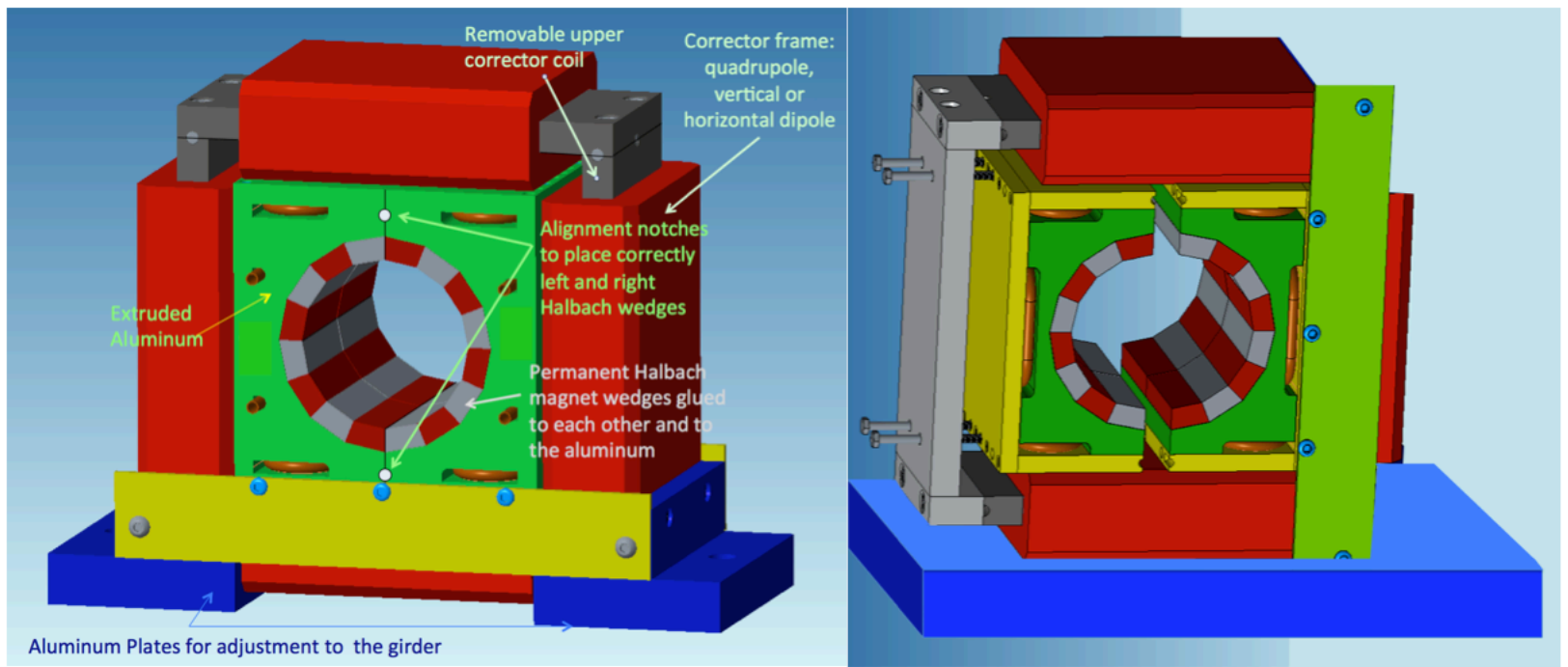

Figure 40: The left-hand magnet is the "BD" combined function magnet, where the asymmetrical choice of permanent magnet thicknesses generates both a dipole and a quadrupole (and the magnetization angles have been chosen to cancel all other harmonics in the ideal case). The right-hand picture shows the "QF" pure quadrupole magnet in the process of being split with screws. This procedure is needed to assemble the magnets around the CBETA vacuum chamber.

For CBETA, an additional online correction is needed to compensate for the material's temperature coefficient and also to provide correction to beam steering and corrections for misalignments errors of the magnet placement. A "window-frame" electromagnetic corrector is placed surrounding the magnet to provide this. The permanent magnet assembly within has $\mu_{r} \sim 1$, so the correction field adds linearly 
onto the main field. A design with these elements is shown in the Picture 40 . Very important procedure for aligning the lower and upper part with the optimum precision is previously shown in Figure 12 .

\subsection{Risk drivers}

\subsubsection{Variability of magnetization from factory}

a) Random errors of block magnetization strength and direction.

Mitigation: These can be corrected by the shimming wires if small enough. For instance $+/-1 \%$ errors in magnetization strength and $+/-0.01 \mathrm{rad}(+/-0.57 \mathrm{deg})$ errors in direction can always be shimmed with 32 wires of up to $63 \mathrm{mil}$ thickness. For larger errors it is possible to use more or thicker wires for a proportionate gain in strength, but the above error sizes are consistent with what was seen in prototyping. There is some averaging, so the "1\%" level block errors produce 25-50 units of total multipole error at the beam radius (swimmable to almost always less than 1 unit in simulation).

b) Overall average magnetization strength of blocks is too small by a few percent. This was observed in measurements of blocks received for the iron-poled magnet: the block-to-block variability was very good but the average was too low.

Mitigation: This can be corrected by designing the magnet for the low end of the strength range. If blocks are then received with a higher average strength, the magnet can be weakened by placing the blocks slightly further away from the center (they may be separated for example by aluminum shimming pieces).

\subsubsection{Temperature coefficient}

a) The $\mathrm{NdFeB}$ material has a temperature coefficient of $-1.1 \mathrm{e}-3 / \mathrm{K}$, meaning the magnet will weaken in higher temperatures (by about $1 \%$ for a $9 \mathrm{~K}$ increase in temperature). There is no obvious way to include temperature compensation material (such as NiFe) into the Halbach magnet due to its geometry with field lines not parallel to the magnetization vectors.

Mitigation: the Halbach magnets will be surrounded by window-frame correctors that can provide at least $+/-2 \%$ of the overall field in quadrupole and dipole. This range would be able to compensate a $20 \mathrm{C}$ (68F) room swinging from $2 \mathrm{C}$ to $38 \mathrm{C}$ ( $36 \mathrm{~F}$ to $100 \mathrm{~F}$ ). The corrections will be calculated from the lowfrequency orbit feedback system in the same way that other slow orbit drifts are compensated. This method has been approved by Chris Mayes (L2 for accelerator physics.

b) It is possible that parts of the magnet will heat up non-uniformly and produce field errors that are not a simple scaling of the dipole + quad field.

Mitigation: the largest source of heat near the magnet is the window-frame corrector coil. A chilled aluminum plate with water-cooling channels has been placed surrounding the NdFeB blocks and inside the window-frame, so that the heat has a much lower resistance path into the cooling water than to the blocks. This will also provide some additional temperature stabilization to the blocks due to the water's regulated temperature.

\subsubsection{Top/bottom alignment after reassembly:}


In order to be able to assemble the magnets around the vacuum chamber pipe (which has protrusions), they must be able to be split into top and bottom halves. Lateral offsets caused by reassembly produce a unit of skew quadrupole at the beam maximum radius for each $5 \mu(0.2 \mathrm{mil})$ displacement and a unit of skew sextupole for each $18 \mu$ (0.7 mil) displacement.

Mitigation: after initial assembly and measurement, a special fixture, as shown in Figure 12, allows firm high accuracy connection of the upper and lower holes. This is provided by additional central mold identical for both type of Halbach magnets and obtained from the 3D printers or extruded aluminum. Holes will be drilled into the centerline between the top and bottom halves, and metal pins fitted tightly into these holes. This is a standard procedure used in e.g. quadrupoles for reliable reassembly.

\subsubsection{Radiation damage}

This design puts the permanent magnet blocks very near the vacuum chamber (within a few $\mathrm{mm}$ ) and they surround it on all sides including the mid-plane where beam losses are most probable. NdFeB is not particularly radiation hard although this varies with material grade.

Mitigation: firstly, quotes have been obtained for the material grades N35SH and N35UH, which have enhanced resistance to demagnetization ("high temperature" grades) and are also not the strongest possible grade (which also tends to be more fragile). CBETA operates at a lower energy than light sources, so synchrotron radiation should be smaller. Beam loss is more concerning: although the strong focusing in the FFAG makes this a less likely place for loss, it will have to be monitored carefully. Permanent magnet wigglers have operated for long periods of time in light sources although these have open mid-planes and some in the literature have shown small amounts of field degradation. A further mitigation is possible by adopting a magnet design where some of the mid-plane is "open" (filled with aluminum rather than $\mathrm{NdFeB}$ ) so radiation on this plane does not directly hit a magnet block. Advice should be sought from wiggler experts.

We have replaced previous permanent magnet material with the permanent $\mathbf{N} 35 \mathrm{UH}, \mathrm{B}_{\mathrm{R}}=1.17-1.21 \mathrm{~T}$, $H_{c}(K O e)=10.8-11.4, H_{C I}(K O e)>25, B_{\max }(M G O e)=33-35, T_{M A X}\left(C^{\circ}\right) \leq 180$, where UH means more resistant to demagnetization and radiation damage. A price of the previous permanent magnet material was for all 220 magnets is $\$ 174,756.30$ while the higher radiation resistance material cost is $\$ 240,678.90$.

\subsubsection{Construction issues}

a) The magnet blocks have large forces between each other during assembly ( 100lbf).

Mitigation: the technique used on the prototypes was to have "dummy" plastic blocks (although they could equally well be non-magnetic metal) initially filling the space, which were replaced one-by-one by magnets (as previously shown in Figure 11). There was also a filler mold piece for the magnet bore. Once all the pieces had been inserted, the 16 wedges of the circular aperture were self-supporting like an arch. However, there were still large forces during assembly: the magnets could be assembled by hand but only just. Some extra tooling might help streamline this, or just buy from a company with experience handling permanent magnets (or wigglers, which are similar).

\section{b) The arrangement of blocks as a whole is an odd shape.}

Mitigation: in the prototypes, a 3D printed plastic mold proved strong enough to surround the magnets provided that it was confined inside an aluminum tube to strengthen against the easiest deformation mode. ABS plastic has survived on the RHIC beam dump to 700Gy of irradiation so could be used in 
production too. Alternatively a custom aluminum extrusion may be affordable when building larger quantities.

\subsection{Drivers for the schedule}

\subsubsection{Permanent magnet blocks initial lead time}

Quotes for the full quantity of wedge-shaped NdFeB blocks from one vendor show an 80-day lead-time. We may want to take delivery of a smaller batch first, however.

\subsubsection{Measurement/shimming cycle}

For each magnet, the main stages are:

- Assembly of Halbach magnet part and bolt it to window-frame corrector;

- Initial rotating coil field measurement;

- Calculate, cut and insert shims using shim holder;

- Second rotating coil measurement;

- [Finish if multipoles are low enough or do another iteration if necessary].

The prototype magnets were removed from the rotating coil in order to insert the shims, so these stages were done on batches of magnets in parallel. It may be faster to shim a single magnet while still on the coil, but this requires the shim wires to be immobilized in their holders so they do not fall out of place when inserted into the magnet, plus $\sim 32$ shim wires to be rapidly cut to different lengths.

\subsection{Drivers of the cost}


Some estimates of the cost for the Halbach magnets (including hardware and labor at BNL rates) have been made and are shown in the chart below:

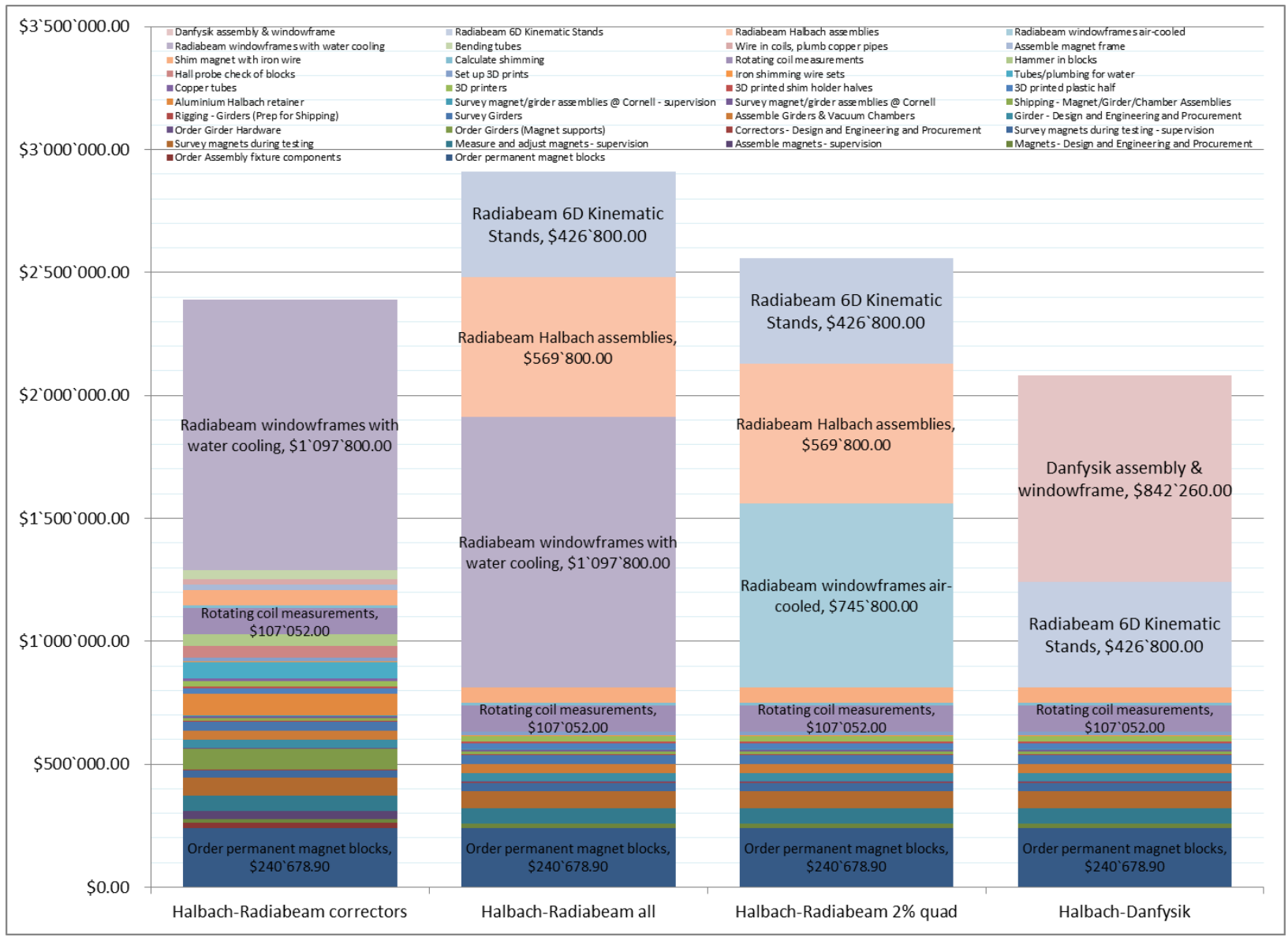

'Radiabeam' quoted either assembling just the window-frame correctors or also the Halbach assemblies plus supporting stands. They would also include an initial 1e-3-level rotating coil measurement to check for errors, although this is not accurate enough to do shimming. There were also two options for the correctors: water-cooled giving $+/-4 \%$ quadrupole adjustment, or air-cooled giving $+/-2 \%$ quadrupole adjustment (and more than sufficient dipole adjustment in both cases). The air-cooled is believed to be sufficient, particularly given the mitigation in $2.1(b)$ is available.

Danfysik provided a quote for the Halbach and window-frame parts, not including the stands, so Radiabeam's estimate is included there.

The permanent magnet blocks themselves are not a major cost driver, being $8-12 \%$ of the total cost. This makes it easier to specify a high temperature resistance grade and purchase spare pieces.

The window-frame correctors (insulated coils and iron) are the most expensive subsystem with $29 \%$ of the total cost when air-cooled and $46 \%$ when water-cooled.

The assembly of the Halbach enclosure from the magnet blocks is the $2^{\text {nd }}$ largest item, from $20-22 \%$ of the total cost.

The kinematic stands seem to be a large cost but Radiabeam may have slightly over specified them pending the final drawings, still they will be significant. 
The labor for rotating coil measurements is large enough to appear at $\sim 5 \%$ of the total. Significant increases in time required for this and shimming may therefore be noticeable.

\section{SCHEDULE}

This is the report from the "RADIABEAM" technology company form Santa Monica:

\subsection{Design and prototyping}

\subsection{First girder}

3.3 Final Production

Due to the schedule demands to test the first sector in Spring 2017, the phases overlap. The first lot will be delivered in completion within 6 months of order and will include 30\% of the ordered quantity. Note that partial shipments will be made available 5 months of order to ease the integration of the magnets. The balance will be available over the subsequent 9 months.

\begin{tabular}{|l|c|c|c|}
\hline Option & & & \\
\hline Air-cooled picture frame magnets & $\$ 3,390.00$ & $\$ 745,800.00$ & \\
\hline Water-cooled picture frame magnets & $\$ 4,990.00$ & $\$ 1,097,800.00$ & \\
\hline Complete Halbach magnet assemblies & $\$ 2,590.00$ & $\$ 569,800.00$ & $\begin{array}{c}\text { Cost in addition to the purchase of one } \\
\text { of the picture frame magnet options }\end{array}$ \\
\hline 6D Kinematic stands & $\$ 1,940.00$ & $\$ 426,800.00$ & \\
\hline
\end{tabular}

The CBETA project critical item is the first girder to be installed in 6 months!

\section{SUMMARY}

We presented a technical design, detail cost estimate, and time schedule of the Halbach type of magnets. We are very confident that they can be immediately used for the existing CBETA project where the base line design of the maximum energy is $E_{\max }=150 \mathrm{MeV}$. We are basing this confidence on the already built twelve prototype Halbach magnets for the previous CBETA design where the maximum energy was $E_{\max }=250 \mathrm{MeV}$. We have been able to develop detailed procedures and necessary fixtures for building them. The magnetic field correction procedures we developed obtain superb quality of the magnetic field in the Halbach magnets.

Our firm opinion that the previous decision to select the Hybrid-iron-dominated magnet (March 2016), driven by the report from the some of the magnet review committee members was not correct. The current information on the Halbach magnets was not available at the time of that review. Some of the committee members were familiar with existing experience in building the iron-dominated magnets. In all prior applications the beam was in the center of the magnets (synchrotrons). The Non-Scaling Fixed Field Alternating Gradient (NS-FFAG) has a significant range of beam positions in the magnets, not a single point. Several committee members posed questions, objections and concerns about Halbach magnets; all have been addressed in this document in the section 1.10 (pages 37-45).

Halbach magnets are a better choice than the Hybrid-iron dominated magnets for the following reasons: 
1. The accelerator physics of NS-FFAG is based on using the combined function magnets to achieve transfer of particles with a large energy range (4 times in energy for CBETA) in a small aperture. This is possible due to the strong focusing and small dispersion function $\Delta x=D_{x} * \delta p / p$. The most efficient way is to use the defocusing combined function magnet but not radially displaced quadrupole. This argument is clearly shown in Figures 2 and 3 (page 5). The misplaced quadrupole (in this case the displacement is $17.3 \mathrm{~mm}$ ) makes a requirement for the good field region equal to $36 \mathrm{~mm}$. The result of this is enormous inefficient magnet with large outside dimensions equal to $66 \mathrm{~cm}$, compared to the $6.8 \mathrm{~cm}$ of the Halbach defocusing magnet.

2. The twelve prototype Halbach magnets obtained superb field quality [Results presented in Tables on pages 31,32, and 33]. The largest measured magnetic multipoles are equal or less than one unit $\left(10^{-4}\right.$ at $\left.1 \mathrm{~cm}\right)$. This is an extraordinary result.

3. Concerns with respect to the effects between the neighboring Halbach magnets, as well as, between Halbach magnets and the corrector iron frames were studied with the OPERA 3D magnetic field simulations. In addition we have performed a detailed harmonic coils measurements of all possible combinations:

3.1. The magnetic field was measured from the two Halbach magnets separated by $6 \mathrm{~cm}$ spacer with and without the other magnet present and with the other magnet twisted $90^{\circ}$. All results confirmed that there is no cross talk between the magnets. There was no measurable difference of the magnetic field in any combination.

3.2. The corrector iron frame was placed around the Halbach magnet and measured with harmonic coil in every possible case: magnetic field without the iron frame, with the frame but without powering the correctors, with powering horizontal corrector, powering the vertical corrector and so on. The effect measured was $0.2 \%$ change of the quadrupole field!!! The Hybrid magnet has significant cross talk.

4. The Halbach magnets allow use of the round vacuum pipe instead of the flat required for the Hybrid iron magnets. This simplifies the construction and the vacuum system removing necessary transitions from the flat to the round pipe required for the horizontal alignment. It also allows use of the four button BPMs instead of 6 button BPMs. The total cost saving of the BPM electronic boards is reduced from $\$ 660,000.0$ to $\$ 330,000$, a saving of $50 \%$.

5. The size and the weight of the magnets are dramatically reduced. This simplifies the installation and is an efficient way of building future ERL's with small magnets.

6. The difference in cost between Halbach and Hybrid iron magnets is 1.2 Million dollars, based on the existing bids.

The first Section shows details of the Halbach magnet design and results from the built CBETA Halbach prototypes. The present base line design with the maximum energy of $150 \mathrm{MeV}$ is used for the Halbach magnet lattice design. Dimensions of the drifts and lengths of the magnets from baseline design were copied (all of it is presented in the section 1.3). The maximum orbit offsets in the focusing Halbach quadrupole are $\Delta x \approx \pm 22 \mathrm{~mm}$ with respect to the center of the magnet. Orbit offset is defined with respect to the central circular orbit (where $x=0$ from the linear magnetic field: $B(x)=B o+G x$ ). The inner 
distance from the center of the focusing quadrupole to the permanent magnet material is $4.5 \mathrm{~cm}$. It is very important to emphasize a difference in orbit offsets for the same gradients and same magnet sizes of the two options. The maximum orbit offset from the center of the focusing Hybrid-iron magnet is $\Delta x \approx 31.5 \mathrm{~mm}$. The same size of the maximum orbit offset of $\Delta x \approx 31.5 \mathrm{~mm}$, from the center of the misplaced defocusing Hybrid quadrupole.

The lattice parameters are presented in the section 1.3.2. They are obtained from tracking electrons through the 3D OPERA magnetic fields. This approach was previously confirmed by excellent agreement between the OPERA 3D predictions and the harmonic coil measurements in the 12 twelve Halbach prototype magnets. The Halbach magnet gradients are adjusted during this procedure, until an agreement of the Twiss parameters between the hardedge model and the 3D OPERA fields tracking are obtained.

The mechanical design including details of the assembly procedures with the fixtures used for all magnets, details and frames for disassembling the magnets if necessary are presented in the section 1.4. Special attention was given to this assembly and disassembly procedure. For example Fig. 12 shows the fixture-frame required for not only assembling the magnet, but also for assuring the best possible alignment between the upper part of the Halbach magnet and the lower part. It is important to emphasize that the alignment pins required for accurate assembling are defined after the upper and lower parts are pressed together making the perfect circular structure. An additional frame for disassembling the magnet at the top of the corrector frame is shown in Figure 14

The shimming procedure is a repeat of the same procedure as in the previous prototype magnets (section 1.7).

The vacuum design for the Halbach magnets is presented in the Section 1.8 with detail picture of the girder pipe design shown in Fig. 17. This is very important advantage of the Halbach magnet vacuum pipe design with respect to the Hybrid-iron magnet design as the vacuum round pipe will simplify the assembly and allow use of 4 button BPMs. This is well defined and proven in the Cornell CESR, and as stated previously this presents a significant savings

The prototype magnet development, design and measurement results are shown in Section 1.9. This is the most important and critical part of this report as it shows the main arguments for the magnet choice. As mentioned above the superb quality of the Halbach magnetic field is shown at pages 31-33.

The most important reason for using the Halbach magnets rather than Hybrid-iron magnets is the lower cost with a difference of at least $\$ 1.2$ million dollars. Details are shown in Section 2.

A copy of the magnet production plan of one of the competitors is shown in Section 3. 


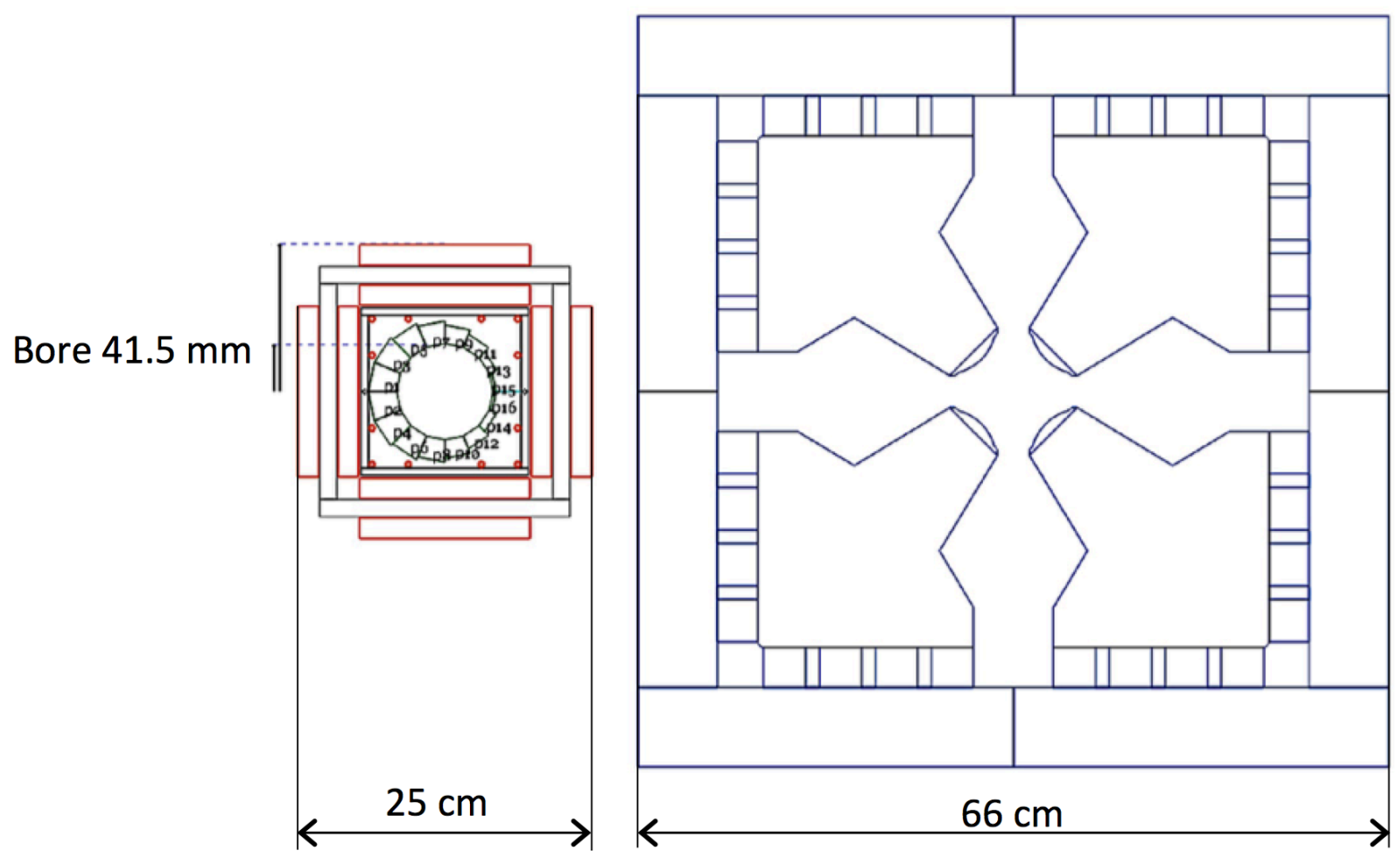

Figure 41: Comparison of the two magnet sizes: Hybrid-iron with Halbach magnet. 